\title{
Retired A Stars and Their Companions. VIII. 15 New Planetary Signals around Subgiants and Transit Parameters for California Planet Search Planets with Subgiant Hosts
}

\author{
Jacob K. Luhn ${ }^{1,5}$ (10, Fabienne A. Bastien ${ }^{1}$ (1) , Jason T. Wright ${ }^{1}$ (i) , John A. Johnson ${ }^{2}$, Andrew W. Howard ${ }^{3}$ (i), and \\ Howard Isaacson ${ }^{4}$ (iD \\ ${ }^{1}$ Department of Astronomy, The Pennsylvania State University, 525 Davey Lab, University Park, PA 16802, USA; jluhn@psu.edu \\ ${ }^{2}$ Institute for Theory and Computation, Harvard-Smithsonian Center for Astrophysics, 60 Garden Street, Cambridge, MA 02138, USA \\ ${ }^{3}$ Department of Astronomy, California Institute of Technology, Pasadena, CA, USA \\ ${ }^{4}$ Astronomy Department, University of California, Berkeley, CA, USA \\ Received 2018 May 17; revised 2018 November 1; accepted 2018 November 5; published 2019 March 20
}

\begin{abstract}
We present the discovery of seven new planets and eight planet candidates around subgiant stars, as additions to the known sample of planets around "retired A stars." Among these are the possible first three-planet systems around subgiant stars, HD 163607 and HD 4917. Additionally, we present calculations of possible transit times, durations, depths, and probabilities for all known planets around subgiant $(3<\log g<4)$ stars, focused on possible transits during the TESS mission. While most have transit probabilities of $1 \%-2 \%$, we find that there are three planets with transit probabilities $>9 \%$.
\end{abstract}

Key words: planets and satellites: detection - planets and satellites: fundamental parameters - techniques: radial velocities

Supporting material: machine-readable tables

\section{Introduction}

The occurrence rate of Jupiter-mass planets has been observed to increase with both metallicity and mass of the host star (Ida \& Lin 2004; Fischer \& Valenti 2005; Bowler et al. 2010; Johnson et al. 2010a). Despite the increased occurrence rates, more massive stars show a paucity in the number of massive planets at short periods, both "hot Jupiters" on very close orbits and even those out to separations of $\sim 1$ au (Johnson et al. 2007a; Sato et al. 2008). As these planets are the easiest to detect via radial velocity (RV) and transit photometry methods, this does not represent an observational bias, indicating that stellar mass plays a large role in shaping the formation and orbital evolution of planets.

Johnson et al. (2006) targeted intermediate-mass evolved stars in an effort to observe and study the properties of planets around stars more massive than the Sun. The stars selected for the survey come from just below a section of the main sequence known as the Hertzprung gap (HG), which lies between the main sequence and the red giant branch. The sample was selected to include mostly intermediate-mass stars $\left(M_{\star} \gtrsim 1.3 M_{\odot}\right)$, often referred to as "retired A-type" stars because they had A spectral types when they were on the main sequence. On the main sequence, these stars are difficult targets for precise RV measurements for two reasons: first, because they rotate rapidly, any absorption features they have are significantly Doppler-broadened and, second, because of their high effective temperatures, they lack strong absorption lines observed in cooler stars. As a result, typical RV surveys avoid main-sequence stars with intermediate to high masses. However, stars of this mass that have left the main sequence become suitable for RV measurements due to their cooler atmospheres and slower rotational velocities, which lead to narrower absorption features in their spectra. The Retired A-star survey

\footnotetext{
${ }^{5}$ NSF Graduate Research Fellow.
}

has been responsible for the discovery of more than 40 exoplanets around subgiant stars to date. ${ }^{6}$

The planets discovered by the Retired A-star survey exhibit two key differences from planets discovered around lower mass mainsequence stars: (1) an increased abundance of giant planets and (2) a decrease in planets with shorter periods. These differences between the planets around more massive stars and those on the main sequence has sparked debate about the true masses of the stars themselves (Lloyd 2011, 2013; Johnson et al. 2013; Johnson \& Wright 2013). The essence of the debate revolves around the statistical likelihood of finding a large population of relatively massive stars in the region of the HR diagram selected for the Retired A-star survey. Different galactic models and using volumelimited versus magnitude-limited samples produce conflicting results for the expected number of massive stars. Furthermore, Schlaufman \& Winn (2013) investigated the kinematics of these stars, concluding that the velocity dispersions were too high for them to in fact be massive stars. Using asteroseismology, Stello et al. (2017) showed that there was indeed an overestimate in mass for stars above $1.6 M_{\odot}$ among their sample of eight stars. More recently, Ghezzi et al. (2018) reanalyzed a subset of the Retired A Star sample and determined atmospheric, rotational, evolutionary, and kinematic parameters, finding errors much lower than the 50\% overestimate suggested by Lloyd (2011, 2013). By accounting for reddening, they find that the velocity dispersions are consistent with those of more massive main-sequence stars with an offset of $0.04 M_{\odot}$, suggesting that these are in fact massive stars. Putting aside the mass argument, because the Retired A-star sample is composed of entirely evolved stars, ${ }^{7}$ the "desert" of short-period planets appears regardless of the true mass of these stars, as there is a noticeable lack of short-period planets around postmain-sequence stars. In fact, there appears to be a pileup of

\footnotetext{
6 http://exoplanets.org

7 Selected based on their position near the Hertzsprung gap.
} 


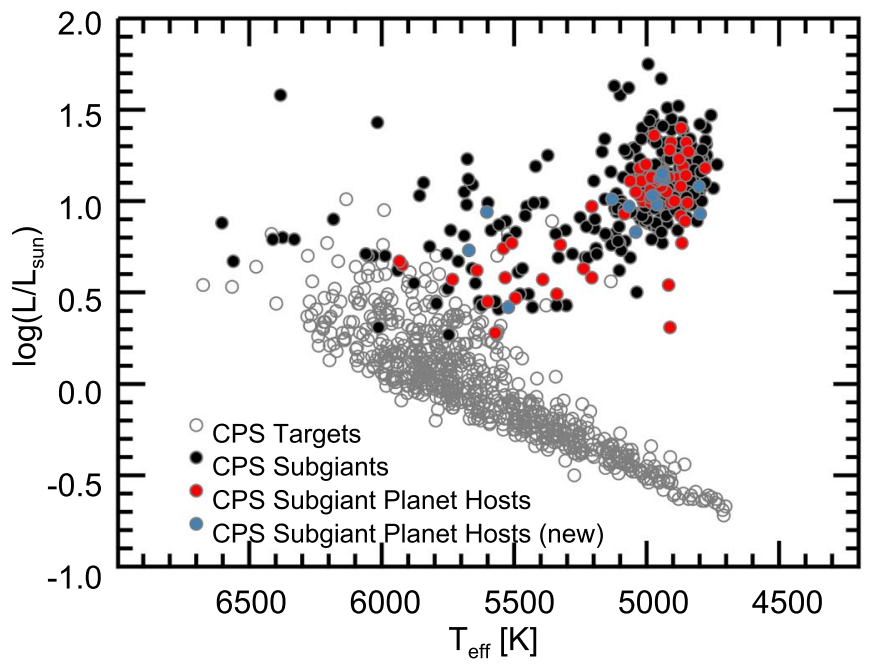

Figure 1. HR diagram showing the sample of subgiants among all CPS stars and the subset of those that are planet-host stars. All CPS target stars with $T_{\text {eff }}$ and $\log \left(L / L_{\odot}\right)$ values from Brewer et al. (2016) are shown in gray. The black points show the subsample of CPS subgiant stars, which are here chosen to be stars with $3.0<\log g<4.0$. The planet-host stars (known and new in this work) are shown in red.

planets around post-main-sequence stars at 1-2 au, all with masses $\sim 1-5 M_{\text {Jup }}$, possibly indicating that these types of planets are those most likely to remain after post-mainsequence stellar evolution while most planets interior to this region are lost due to tidal capture (Villaver \& Livio 2009).

Given the lack of short-period planets around subgiants, there are few planets around subgiants that are easily detectable by transit surveys. Despite this, a handful of short-period planets with subgiant hosts have been discovered by Kepler (Borucki et al. 2010), namely Kepler 435 b (Borucki et al. 2010); Kepler 56 b,c (Borucki et al. 2011); Kepler 108 b,c, 278 b,c, 391 b,c (Rowe et al. 2014); Kepler 432 b (Ciceri et al. 2015); and Kepler 637 b, 815 b, 1270 b, 774 b, 1004 b, 1394 b, and 643 b (Morton et al. 2016) as well as one planet in the KELT survey, KELT-11 b (Pepper et al. 2017). ${ }^{8}$ Unfortunately, the Kepler subgiants are generally too faint and were lower priority than dwarfs for RV follow-up and so we do not have measured masses for most of these planets. ${ }^{9}$ However, given the large sample of existing RV subgiants with known planets, we have the opportunity to examine the transit probabilities for these lower priority transit search targets, in the context of future transit surveys, particularly on the upcoming TESS mission (Ricker et al. 2014). Observing transits of existing RV planets has a number of advantages, the biggest being removing the $\sin i$ degeneracy for the masses of these planets. Additionally, transits provide a model-independent measure of the stellar density, which could be useful for confirming the masses of the host stars. Another benefit is the tendency of RV surveys to target bright stars, which enables easier ground-based follow-up and provides targets for transmission spectroscopy, for which we have very few studies of temperate, long-period Jupiter-sized planets (Kane et al. 2009). Despite large transit surveys (KELT, HAT, Kepler, K2) in this time, long-period Jupiters with hosts bright enough for transmission spectroscopy remain scarce. With

\footnotetext{
8 KELT-6 b (Collins et al. 2014) also orbits what appears to be a very slightly evolved subgiant.

9 Those with measured masses are Kepler 56 b,c (Otor et al. 2016) and Kepler 432 c (Quinn et al. 2015).
}

these ideas in mind, we present transit parameters for subgiant stars already observed by the California Planet Search (CPS; Howard et al. 2010) RV survey.

In Section 2, we describe our sample of subgiant stars, RV measurements, and stellar properties. We then present the discovery of 15 new planetary signals around subgiant stars and several stellar companions, spanning a spectrum of secure detections. Section 3 contains those that we determine to be secure planet detections. Section 4 lists those that are "planet candidates" to better illustrate the varying degrees of security. We include all probable planet candidates in the interest of listing the possible transit times for planets around subgiant hosts. Section 5 contains a list of the stellar companions. The new planetary signals in this work increase the sample of RV planets around subgiants by more than $25 \%$. In Section 6 we present the transit parameters for these stars. Despite the typically small transit probability of $1 \%-2 \%$, it is likely, given the sample size of 85 planets, that several do indeed transit. Finally, we present a summary and our conclusion in Section 7.

\section{Observations}

\subsection{Sample Selection and Stellar Properties}

Our sample is composed of stars observed as part of the CPS with $3.0<\log g<4$.0. From this sample of over 400 stars, we have identified those that are known to host planets, as identified in either www.exoplanets.org or www.exoplanet.eu. This list comprises 42 stars in our sample. Figure 1 shows an HR diagram of the entire CPS sample, the subset of CPS subgiant stars, and the planet-host stars in our sample.

All stellar properties come from Brewer et al. (2016; erratum Brewer et al. 2017 cited as B17 hereafter), who used onedimensional LTE model spectra to fit to a star's observed spectrum to determine effective temperatures, metallicities, surface gravities, and elemental abundances. In conjunction with Hipparcos parallaxes and $V$-band magnitudes, these spectral measurements were used to derive masses, radii, and luminosities. Their iterative fitting technique and improved line list correct for systematic discrepancies in $\log g$ between spectroscopy and asteroseismology (Huber et al. 2013; Bastien et al. 2014) and their spectroscopic methods are now consistent with the values of $\log g$ obtained from asteroseismology (Brewer et al. 2015). The stellar properties for the stars in this sample are given in Table 2. We note that while Ghezzi et al. (2018) determined masses and radii with smaller uncertainties than Brewer et al. (2016) for a subset of the Retired A sample, more than $25 \%$ of the stars in this work were not reanalyzed by Ghezzi et al. (2018). Since the stellar parameters are largely consistent within errors between Ghezzi et al. (2018) and Brewer et al. (2016), we use stellar properties from Brewer et al. (2016) for consistency. ${ }^{10}$

\subsection{Spectra and RV Measurements}

Observations were taken at Keck Observatory using the High Resolution Echelle Spectrometer (HIRES) with a resolution of

\footnotetext{
${ }^{10}$ The radius measurement of HD 193342 is the only parameter for which the two samples are not consistent within the errors, with radius $6 R_{\odot}$ from Brewer et al. (2016) and $8.5 R_{\odot}$ from Ghezzi et al. (2018). Despite this large discrepancy, the radius difference will only affect the predicted transit parameters for HD $193342 \mathrm{~b}$, which is unlikely to transit $(0.27 \%$ transit probability). Using the radius from Ghezzi et al. (2018) would result in a slightly increased transit probability $(\sim 0.4 \%)$ as well as a smaller transit depth and longer transit durations if it does indeed transit.
} 
Table 1

Summary of Additional Non-Keck HIRES Velocities

\begin{tabular}{|c|c|c|c|c|}
\hline Star & Telescope & Instrument & $N_{\text {obs }}$ & References \\
\hline HD 1502 & Harlan J. Smith Telescope & TCS & 25 & Johnson et al. (2011) \\
\hline HD 1502 & Hobby-Eberly Telescope & HRS & 20 & Johnson et al. (2011) \\
\hline HD 159868 & Anglo-Australian Telescope & UCLES & 47 & Wittenmyer et al. (2012) \\
\hline HD 192699 & Lick Observatory & Hamilton spectrometer & 34 & Johnson et al. (2007b) \\
\hline HD 114613 & Anglo-Australian Telescope & UCLES & 222 & Wittenmyer et al. (2014) \\
\hline HD 38801 & Subaru & High Dispersion Spectrograph & 11 & Harakawa et al. (2010) \\
\hline HD 181342 & CTIAO $1.5 \mathrm{~m}$ & CHIRON & 11 & Jones et al. (2016) \\
\hline HD 181342 & CTIAO $2.2 \mathrm{~m}$ & FEROS & 20 & Jones et al. (2016) \\
\hline HD 181342 & Anglo-Australian Telescope & UCLES & 5 & Wittenmyer et al. (2011) \\
\hline HD 5608 & OAO $1.88 \mathrm{~m}$ & HIDES & 43 & Sato et al. (2012) \\
\hline HD 10697 & Harlan J. Smith Telescop & TCS & 32 & Wittenmyer et al. (2009) \\
\hline HD 10697 & Hobby-Eberly Telescope & HRS & 40 & Wittenmyer et al. (2009) \\
\hline HD 210702 & Lick Observatory & Hamilton spectrometer & 29 & Johnson et al. (2007a) \\
\hline HD 210702 & OAO $1.88 \mathrm{~m}$ & HIDES & 36 & Sato et al. (2012) \\
\hline HD 214823 & Observatoire de Haute-Provence $1.93 \mathrm{~m}$ & SOPHIE & 13 & Díaz et al. (2016) \\
\hline HD 214823 & Observatoire de Haute-Provence $1.93 \mathrm{~m}$ & SOPHIE + & 11 & Díaz et al. (2016) \\
\hline HD 93396 & Lick Observatory (APF) & Levy Spectrometer & 15 & Pepper et al. (2017) \\
\hline
\end{tabular}

$R \approx 55,000$. For a $V=8$ magnitude star, the exposure required is $90 \mathrm{~s}$ to reach a signal-to-noise ratio of 190 at $5800 \AA$ A RVs are calculated using the iodine-cell calibration technique and the forward-modeling procedure described in Butler et al. (1996) and later Howard et al. (2011). For several stars that were known planet hosts, we included the non-Keck RV measurements as published with the planet discovery. These stars and the instruments used are briefly described below with a summary included in Table 1.

HD 1502-In addition to Keck/HIRES observations HD 1502 was also observed using the Tull Coude Spectrograph (Tull et al. 1995) on the Harlan J. Smith Telescope as well as the High Resolution Spectrograph (Tull 1998) on the Hobby-Eberly Telescope (HET; Ramsey et al. 1998), both of which have $R=60,000$. Differential RVs were computed using the Austral $I_{2}$-data modeling algorithm (Endl et al. 2000). The velocities are given in Table 2 of Johnson et al. (2011).

HD 159868 - HD 159868 was observed mainly with the Anglo-Australian Telescope (AAT) (Jones et al. 2002) using the UCLES echelle spectrograph. RVs are given in Table 2 of Wittenmyer et al. (2012).

HD 192699-HD 192699 was also observed with Lick Observatory's Shane $3 \mathrm{~m}$ and $0.6 \mathrm{~m}$ Coude Auxiliary Telescopes, which feed into the Hamilton spectrometer (Vogt 1987). The velocities for this star are given in Table 3 of Johnson et al. (2007b).

HD 114613-HD 114613 is another star that was observed as part of the Anglo-Australian Planet Search and has RV measurements from the UCLES echelle spectrograph (Diego et al. 1990). The RVs are given in Table 1 of Wittenmyer et al. (2014).

HD 38801-Additional RV observations of HD 38801 come from the high dispersion spectrograph on the $8.2 \mathrm{~m}$ Subaru Telescope (Noguchi et al. 2002). The RV measurements can be found in Table 2 of Harakawa et al. (2010).

HD 181342-HD 181342 (also called HIP 95124) has a number of observations from various instruments, including two telescopes at the Cerro Tololo Inter-American Observatory: the $1.5 \mathrm{~m}$ telescope using the CHIRON spectrograph (Tokovinin et al. 2013) and the $2.2 \mathrm{~m}$ telescope using the FEROS spectrograph (Kaufer et al. 1999). In addition, it was observed as part of the Pan Pacific Planet Search (Wittenmyer et al. 2011) using the UCLES spectrograph (Diego et al. 1990). All velocities from these instruments are listed in Table A.4 of Jones et al. (2016).

HD 5608-HD 5608 was observed as part of the Okayama Planet Search Program (Sato 2005), which uses the HIgh Dispersion Echelle Spectrograph (HIDES) on the $1.88 \mathrm{~m}$ telescope at Okayama Astrophysical Observatory (OAO). The velocities are given in Table 2 of Sato et al. (2012).

HD 10697-HD 10697 was also observed using the two telescopes at McDonald observatory: HET and the $2.7 \mathrm{~m}$ Harlan J. Smith telescope. The velocities are given in the electronic version of Table 10 in Wittenmyer et al. (2009).

HD 210702-HD 210702 has observations from both Lick Observatory and OAO. The velocities from Lick are given in Johnson et al. (2007a). The velocities from OAO are given in Table 8 of Sato et al. (2012).

HD 214823-HD 214823 was observed using ELODIE and SOPHIE/SOPHIE + instruments on the $1.93 \mathrm{~m}$ telescope at Observatoire de Haute-Provence. Given ELODIE's large instrumental uncertainty $\left(15-30 \mathrm{~m} \mathrm{~s}^{-1}\right)$ and that there are only five measurements from ELODIE we use only the RVs from SOPHIE and upgraded SOPHIE+ instruments. This accounts for an additional 24 observations for this star. These velocities are given in Díaz et al. (2016).

HD 93396-HD 93396 (KELT-11) was observed using the Levy spectrograph on the Automated Planet Finder (APF) telescope at Lick Observatory. The velocities are are given in Table 6 of Pepper et. al (2017).

\section{Seven New Planets around Subgiants}

Here we present the new planets discovered around subgiant stars. All RV time series for every star used in this work are given in Table 4. We note that our quoted values of $\chi^{2}$ from the fitting procedure RVLIN throughout this paper are significantly larger than 1. The reason is because we have use only the reported internal measurement errors in our fits. Previous works have inflated individual measurement errors by including a "jitter" term in quadrature to account for increased RV variations due to intrinsic stellar variability (Wright 2005). However, given the large known uncertainty in jitter estimates in Wright (2005), we do not follow this approach. Finally, all stellar parameters quoted 
here come from B17 and were used to obtain planet parameters where necessary (planet mass, transit probabilities, etc.).

The majority of planets described below have periods $>300$ days. At these periods, the greatest concern for the planet validity is stellar activity cycles that operate on similar timescales. To avoid misidentifying a stellar activity cycle as a planet, we have examined the simultaneous $s$-index time series for each of these planets. Except for HD 180053, none of the new planet hosts in this work (including planet candidates in Section 4) show significant activity cycles that correlate with the RVs. Furthermore, none of the stars show evidence of noncycling activity that correlates with the RV measurements. Thus, we are confident that we have identified planet signals rather than activity-induced RVs.

Another timescale of importance is the stellar rotation timescale, which can be hundreds of days for giant stars. For the subgiants in this work we expect that the rotation periods are all much less than the planet periods given that most planet periods here are $>300$ days. While it is possible that some of the planet periods are on timescales similar to the rotation timescales, the coherence and amplitudes of these signals make it unlikely that they are due to rotationally modulated inhomogeneities.

Lastly, we have examined the spectral window functions of the RV time series as described in Dawson \& Fabrycky (2010) to be certain that none of the planets in this work are a result of sparsely sampled data that could introduce spurious periodicity that lead to false peaks in the periodogram. We find that none of the planets in this work correspond to significant peaks in the spectral window function.

We have ordered the new planets in this paper in descending order of detection security, with the most secure detections first. In general we based our threshold for planet status as meeting three main criteria. First, we need to have observed a full period such that $N_{\mathrm{p}}>1$, where $N_{\mathrm{p}}$ is the number of periods observed, found by simply dividing the baseline of the time series by the best-fit period. We generally wish $N_{\mathrm{p}} \gg 1$ to be certain about a planet but our threshold of 1 makes it necessary to have observed two instances where the velocities have turned over. Second, we examine the false alarm probability (FAP) and choose a 1\% FAP threshold. Third, and most important, is the ratio of the semiamplitude to the rms, $K / \sigma$. This ratio alone indicates the ability to detect the planet in a single measurement. We expect then that our ability to detect a planet scales with the number of observations and define a detection threshold $D$

$$
D \equiv \frac{K}{\sigma} \sqrt{N-M}
$$

where $N$ is the number of observations and $M$ is the number of free parameters in the planet fit (six for a one-planet fit plus five for each additional planet and one additional free parameter if the fit includes a linear trend). For a " $10 \sigma$ detection," $D$ must be greater than $10 .^{11}$

\subsection{A $1.7 M_{\mathrm{Jup}}$ Planet around HD 72490}

HD 72490 is a G5 subgiant with $V=7.83, B-V=0.95$ and a parallax-based distance of $124.22 \mathrm{pc}$ (Wenger et al. 2000).

\footnotetext{
${ }^{11}$ We find that these thresholds combine such that a single threshold of $D>17$ is able to distinguish planet versus candidate. Although arbitrary, this singular threshold is able to match our intuition.
}

It has a radius of $\sim 5 R_{\odot}$, an effective temperature $T_{\text {eff }}=4934 \mathrm{~K}$, and surface gravity $\log g=3.210$. A summary of its stellar parameters can be found in Table 2 .

The initial observations of this star began in late 2007 and finished in 2014, with only two observations since 2014. It was identified in Butler et al. (2017) as having a planet candidate. The best-fit Keplerian orbital solution yields an orbital period of $P=858 \pm 12$ days $(2.35 \mathrm{yr})$, velocity semiamplitude $K=33.5 \pm 1.5 \mathrm{~m} \mathrm{~s}^{-1}$, and eccentricity $e=0.124 \pm 0.046$. From the stellar mass $M_{\star}=1.21 M_{\odot}$ we derive the minimum mass of the planet $m_{\mathrm{p}} \sin i=1.768 \pm 0.080 M_{\mathrm{Jup}}$ and semimajor axis $a=1.88 \pm 0.17 \mathrm{au}$. The full set of orbital parameters and corresponding uncertainties are given in Table 3. The time series showing the signal of HD $72490 \mathrm{~b}$ is shown in the left panel of Figure 2. A search for a possible second planet yielded nothing of significance, which is supported by the periodogram of the raw RV data along with the periodogram of the data with the best-fit model of HD $72490 \mathrm{~b}$ subtracted out (shown in the right panel of Figure 2). For HD $72490 \mathrm{~b}$, the preliminary next predicted transit is BJD $2459074.350 \pm 41.296$ (2020 December 8). Its most recent predicted transit was in early April of 2018 and before that in late November of 2015, missing its $K 2$ Campaign 5 observations by a mere 4 months.

\subsection{A Jupiter-mass Planet around HD 94834}

HD 94834 is a K1 subgiant with $V=7.61, B-V=0.99$ (Wenger et al. 2000). It has effective temperature $T_{\text {eff }}=$ $4798 \mathrm{~K}$, and surface gravity $\log g=3.22$. A summary of its stellar parameters can be found in Table 2 .

Observations of this star span roughly $8.5 \mathrm{yr}$ with the majority of observations between 2010 and 2012. The final five observations demonstrate the periodicity of the signal. HD 94834 was identified in Butler et al. (2017) as having a planet candidate. Our best-fit orbital solution shows a period of $1576 \pm 76$ days $(\sim 4.3 \mathrm{yr})$, velocity semiamplitude $K=$ $20.7 \pm 2.9 \mathrm{~m} \mathrm{~s}^{-1}$, and eccentricity $e=0.14 \pm 0.10$. The minimum mass of this planet is $m_{\mathrm{p}} \sin i=1.192 \pm 0.017 M_{\mathrm{Jup}}$ with a semimajor axis $a=2.74 \pm 0.19$ au. The full set of orbital parameters are given in Table 3. Figure 3 shows the time series of HD $94834 \mathrm{~b}$, with the initial and final periodogram for this system.

\subsection{A Jupiter Orbiting HD 14787}

HD 14787 is a G5 subgiant star with $V=7.63$ and $B-V=0.93$ (Wenger et al. 2000). It has a mass of $M_{\star}=$ $1.43 M_{\odot}$, surface gravity $\log g=3.23$, and $T_{\text {eff }}=4946 \mathrm{~K}$. A summary of its stellar parameters can be found in Table 2 . Observations of this star span about $9 \mathrm{yr}$ starting in 2007. The best-fit Keplerian solution yields an orbital period of $676.6 \pm 8.1$ days, velocity semiamplitude $20.7 \pm 1.3 \mathrm{~m} \mathrm{~s}^{-1}$, and eccentricity 0.155 . It is only slightly more massive than Jupiter-mass with a minimum mass of $1.121 \pm 0.069 M_{\text {Jup }}$. The full set of orbital parameters can be found in Table 3. We show in Figure 4 the time series for HD $14787 \mathrm{~b}$ as well as the periodogram before and after subtracting out HD $14787 \mathrm{~b}$.

\subsection{A Jupiter Orbiting HD 13167}

HD 13167 is a G3 subgiant star with $V=8.34$ and $B-V=$ 0.65 (Wenger et al. 2000). It has a mass of $M_{\star}=1.35 M_{\odot}$, surface gravity $\log g=3.72$, and $T_{\text {eff }}=5671 \mathrm{~K}$. A summary of 
Table 2

Stellar Parameters for Subgiants with Known Companions

\begin{tabular}{|c|c|c|c|c|c|c|c|c|c|c|}
\hline (1) & $\begin{array}{c}\text { R.A. } \\
\text { (deg) } \\
(2)\end{array}$ & $\begin{array}{c}\text { Decl. } \\
\text { (deg) } \\
(3)\end{array}$ & $\begin{array}{c}V \\
(\mathrm{mag}) \\
(4)\end{array}$ & $\begin{array}{c}\text { Mass } \\
\left(M_{\odot}\right) \\
(5)\end{array}$ & $\begin{array}{c}\text { Radius } \\
\left(R_{\odot}\right) \\
(6)\end{array}$ & $\begin{array}{l}T_{\text {eff }} \\
(\mathrm{K}) \\
(7)\end{array}$ & $\begin{array}{c}\log g \\
(8)\end{array}$ & $\begin{array}{c}\log R_{\mathrm{HK}}^{\prime} \\
\quad(9)\end{array}$ & $\begin{array}{r}S_{\mathrm{HK}} \\
(10)\end{array}$ & $\begin{array}{c}\sigma_{\mathrm{RV}} \\
\left(\mathrm{m} \mathrm{s}^{-1}\right) \\
(11)\end{array}$ \\
\hline HD 10697 & 26.23260 & 20.08315 & 6.27 & 1.13 & 1.79 & 5600 & 3.96 & -5.04 & 0.16 & 7.856 \\
\hline HD 38529 & 86.64547 & 1.16819 & 5.95 & 1.41 & 2.56 & 5541 & 3.77 & -5.03 & 0.16 & 9.531 \\
\hline HD 114613 & 198.01328 & -37.80302 & 4.85 & 1.27 & 2.14 & 5641 & 3.87 & -5.08 & 0.15 & 5.675 \\
\hline HD 117176 & 202.10753 & 13.77879 & 4.97 & 1.09 & 1.89 & 5495 & 3.95 & -5.12 & 0.14 & 3.974 \\
\hline HD 159868 & 264.74802 & -43.14551 & 7.24 & 1.19 & 2.13 & 5534 & 3.92 & -5.02 & 0.16 & 5.485 \\
\hline HD 175541 & 283.92035 & 4.26533 & 8.02 & 1.39 & 4.19 & 5013 & 3.33 & -5.18 & 0.14 & 6.516 \\
\hline HD 190228 & 300.75323 & 28.30686 & 7.30 & 1.14 & 2.51 & 5238 & 3.72 & -4.98 & 0.17 & 4.314 \\
\hline HD 1502 & 4.82111 & 14.05475 & 8.36 & 1.46 & 4.67 & 4947 & 3.18 & -5.15 & 0.14 & 11.328 \\
\hline HD 3404 & 9.19505 & -24.50094 & 7.94 & 1.17 & 2.05 & 5339 & 3.81 & -5.05 & 0.16 & 2.120 \\
\hline HD 4313 & 11.41816 & 7.84502 & 7.83 & 1.63 & 5.14 & 4943 & 3.24 & -5.28 & 0.12 & 4.695 \\
\hline HD 5319 & 13.75583 & 0.78956 & 8.05 & 1.27 & 4.06 & 4871 & 3.26 & -5.28 & 0.12 & 6.802 \\
\hline HD 5608 & 14.55925 & 33.95089 & 5.99 & 1.53 & 5.14 & 4877 & 3.19 & -5.39 & 0.10 & 8.206 \\
\hline HD 6019 & 15.41506 & 7.30529 & 7.75 & 1.12 & 4.98 & 5020 & 3.08 & -5.07 & 0.16 & 4.390 \\
\hline HD 8375 & 20.90615 & 34.24589 & 6.28 & 1.62 & 3.75 & 5207 & 3.66 & -4.94 & 0.19 & 7.937 \\
\hline HD 10011 & 24.35865 & -15.99768 & 7.99 & 1.50 & 4.32 & 5025 & 3.28 & -5.13 & 0.14 & 4.961 \\
\hline HD 10212 & 25.20706 & 45.01896 & 8.13 & 1.18 & 4.34 & 4907 & 3.14 & -5.21 & 0.14 & 28.302 \\
\hline HD 10442 & 25.47143 & 2.70438 & 7.84 & 1.01 & 1.97 & 4912 & 3.19 & -5.18 & 0.14 & 5.610 \\
\hline HD 11970 & 29.60127 & 40.91367 & 8.23 & 1.24 & 3.74 & 5084 & 3.44 & -5.10 & 0.15 & 8.935 \\
\hline HD 13167 & 32.05743 & -24.69541 & 8.34 & 1.35 & 2.39 & 5671 & 3.72 & -5.12 & 0.14 & 3.985 \\
\hline HD 14787 & 35.80856 & 10.83675 & 7.63 & 1.43 & 5.01 & 4946 & 3.23 & -5.18 & 0.14 & 5.309 \\
\hline HD 18015 & 43.36336 & -8.84802 & 7.89 & 1.49 & 3.13 & 5603 & 3.64 & -4.94 & 0.17 & 8.407 \\
\hline HD 18667 & 45.03415 & 0.23543 & 8.34 & 1.05 & 3.95 & 4855 & 3.08 & -5.22 & 0.13 & 5.167 \\
\hline HD 18742 & 45.04440 & -20.80261 & 7.81 & 1.36 & 5.13 & 4940 & 3.09 & -5.22 & 0.13 & 5.833 \\
\hline HD 21340 & 51.40204 & -27.27249 & 7.40 & 1.55 & 6.32 & 4908 & 3.07 & -5.22 & 0.13 & 5.678 \\
\hline HD 28678 & 67.85606 & 4.57530 & 8.38 & 1.53 & 6.48 & 4972 & 3.06 & -5.27 & 0.13 & 5.780 \\
\hline HD 30856 & 72.57442 & -24.36884 & 7.91 & 1.17 & 4.40 & 4895 & 3.20 & -5.23 & 0.13 & 5.518 \\
\hline HD 33142 & 76.89809 & -13.98648 & 7.96 & 1.41 & 4.45 & 4978 & 3.40 & -5.18 & 0.14 & 5.165 \\
\hline HD 38801 & 86.99657 & -8.32770 & 8.26 & 1.29 & 2.41 & 5207 & 3.77 & -5.02 & 0.17 & 14.413 \\
\hline HD 45410 & 97.69628 & 58.16263 & 5.86 & 1.44 & 5.20 & 4938 & 3.19 & -5.29 & 0.12 & 3.501 \\
\hline HD 51272 & 104.59510 & 37.98798 & 7.78 & 1.73 & 7.00 & 4870 & 3.08 & -5.19 & 0.15 & 23.076 \\
\hline HD 72490 & 128.40271 & 13.55079 & 7.82 & 1.21 & 4.96 & 4934 & 3.21 & -5.19 & 0.14 & 6.177 \\
\hline HD 73534 & 129.81584 & 12.96037 & 8.23 & 1.16 & 2.58 & 4917 & 3.60 & -5.24 & 0.13 & 4.176 \\
\hline HD 75784 & 133.09976 & 13.23344 & 7.84 & 1.26 & 3.40 & 4867 & 3.46 & -5.24 & 0.13 & 4.603 \\
\hline HD 88133 & 152.53198 & 18.18687 & 8.01 & 1.26 & 2.20 & 5392 & 3.88 & -5.18 & 0.14 & 4.376 \\
\hline HD 93396 & 161.70726 & -9.39902 & 8.04 & 1.46 & 2.83 & 5326 & 3.74 & -4.90 & 0.20 & 6.886 \\
\hline HD 94834 & 164.31297 & 24.14278 & 7.60 & 1.11 & 4.20 & 4798 & 3.22 & -5.22 & 0.14 & 6.319 \\
\hline HD 95089 & 164.69890 & 1.72922 & 7.92 & 1.54 & 5.08 & 4918 & 3.24 & -5.21 & 0.13 & 6.798 \\
\hline HD 96063 & 166.18523 & -2.51322 & 8.21 & 1.37 & 4.75 & 5020 & 3.33 & -5.12 & 0.15 & 5.197 \\
\hline HD 96167 & 166.31279 & -10.29130 & 8.09 & 1.27 & 1.94 & 5733 & 3.99 & -5.16 & 0.14 & 4.298 \\
\hline HD 97601 & 168.63914 & 52.94690 & 7.46 & 1.64 & 4.66 & 5062 & 3.34 & -4.67 & 0.33 & 20.386 \\
\hline HD 98219 & 169.44814 & -23.97542 & 8.05 & 1.41 & 4.60 & 4925 & 3.36 & -5.21 & 0.14 & 5.914 \\
\hline HD 99706 & 172.12589 & 43.96658 & 7.65 & 1.46 & 5.52 & 4862 & 3.09 & -5.25 & 0.13 & 11.989 \\
\hline HD 102956 & 177.84380 & 57.64074 & 7.86 & 1.66 & 4.55 & 4985 & 3.38 & -5.07 & 0.18 & 6.300 \\
\hline HD 106270 & 183.40535 & -9.51338 & 7.58 & 1.39 & 2.66 & 5509 & 3.72 & -4.90 & 0.19 & 10.294 \\
\hline HD 108863 & 187.58296 & 21.94824 & 7.71 & 1.59 & 5.74 & 4878 & 3.07 & -5.27 & 0.13 & 6.186 \\
\hline HD 112988 & 195.04286 & 34.99842 & 7.76 & 1.04 & 5.25 & 4852 & 3.25 & -5.21 & 0.13 & 8.404 \\
\hline HD 125390 & 214.53883 & 38.96698 & 8.21 & 1.36 & 6.47 & 4850 & 3.13 & -5.21 & 0.14 & 8.013 \\
\hline HD 125607 & 214.86337 & 37.60997 & 8.09 & 1.47 & 4.31 & 4985 & 3.34 & -5.16 & 0.14 & 17.358 \\
\hline HD 131496 & 223.34595 & 18.23540 & 7.80 & 1.34 & 4.44 & 4846 & 3.18 & -5.33 & 0.12 & 7.183 \\
\hline HD 238433 & 228.40825 & 57.19163 & 8.37 & 1.37 & 5.09 & 4936 & 3.13 & -5.14 & 0.15 & 9.793 \\
\hline HD 142091 & 237.80804 & 35.65738 & 4.79 & 1.50 & 4.85 & 4871 & 3.26 & -5.31 & 0.12 & 2.838 \\
\hline HD 145428 & 242.96356 & -25.88357 & 7.73 & 1.02 & 5.70 & 4779 & 3.13 & -5.29 & 0.13 & 4.440 \\
\hline HD 148284 & 246.45174 & 30.26513 & 9.01 & 1.07 & 1.48 & 5572 & 3.97 & -5.14 & 0.14 & 3.085 \\
\hline HD 152581 & 253.43159 & 11.97375 & 8.38 & 1.30 & 5.14 & 5027 & 3.29 & -5.14 & 0.15 & 4.730 \\
\hline HD 163607 & 268.41873 & 56.39196 & 8.00 & 1.12 & 1.76 & 5522 & 3.97 & -5.01 & 0.16 & 2.878 \\
\hline HD 180053 & 288.40088 & 34.91454 & 7.93 & 1.75 & 4.06 & 5131 & 3.54 & -4.73 & 0.31 & 13.381 \\
\hline HD 180902 & 289.82379 & -23.55816 & 7.78 & 1.41 & 4.16 & 4961 & 3.36 & -5.13 & 0.15 & 1.944 \\
\hline HD 181342 & 290.26764 & -23.61957 & 7.55 & 1.69 & 4.71 & 4945 & 3.28 & -5.31 & 0.12 & 10.681 \\
\hline HD 185269 & 294.29892 & 28.49986 & 6.67 & 1.30 & 2.00 & 5923 & 3.92 & -5.03 & 0.15 & 7.018 \\
\hline HD 192699 & 304.02502 & 4.58079 & 6.44 & 1.38 & 4.41 & 5041 & 3.25 & -5.26 & 0.12 & 8.623 \\
\hline HD 193342 & 304.25226 & 56.90459 & 8.07 & 1.69 & 6.01 & 4913 & 3.19 & -5.21 & 0.14 & 7.438 \\
\hline HD 195787 & 307.64395 & 58.28724 & 7.63 & 1.40 & 4.87 & 4961 & 3.26 & -5.16 & 0.14 & 3.395 \\
\hline
\end{tabular}


Table 2

(Continued)

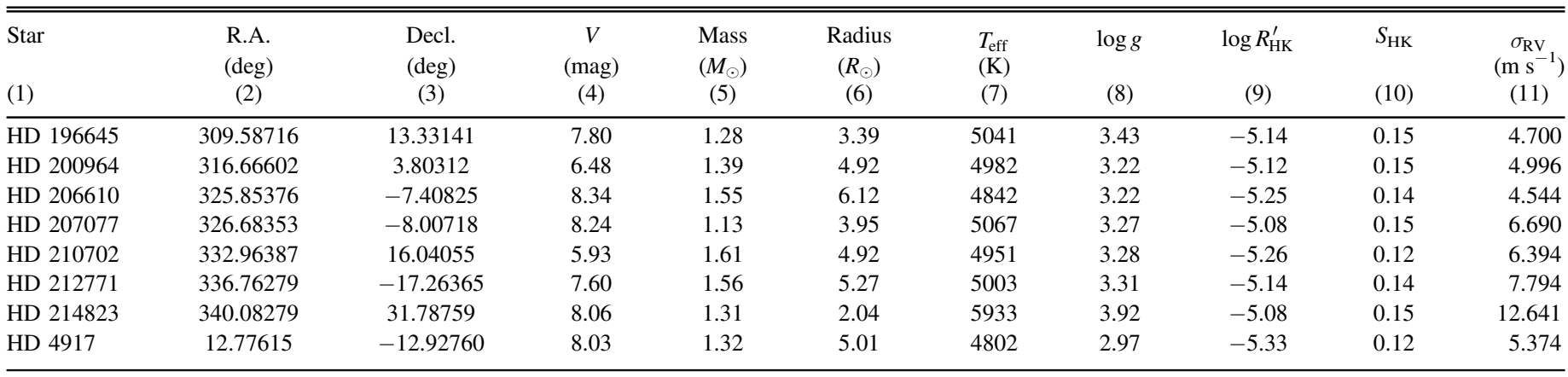

Note. Host star parameters for all planets listed in Table 5. Column 1 lists the star name. Columns 2 and 3 give the coordinates. Data in columns 4-10 come from Brewer et al. (2016). Column 4 gives the $V$-band magnitude. Columns 5, 6, and 7 list the mass, radius, and effective temperature of the star. Column 8 gives the spectroscopic surface gravity. Columns 9 and 10 give two measures of chromospheric activity. We note that for most subgiants in this sample, the log $R_{\mathrm{HK}}^{\prime}$ value is not calibrated, which is why we have also included $S_{\mathrm{HK}}$. Finally, column 11 gives the measured RV rms after subtracting the planetary signal.

(This table is available in machine-readable form.)

its stellar parameters can be found in Table 2. Observations of this star span about $8 \mathrm{yr}$ starting in late 2007. The best-fit Keplerian solution yields an orbital period of $2613 \pm 17$ days, and velocity semiamplitude $48.2 \pm 2.7 \mathrm{~m} \mathrm{~s}^{-1}$ on a fairly eccentric orbit $(e=0.563 \pm 0.033)$. It has a minimum mass of $3.31 \pm 0.16 M_{\mathrm{Jup}}$. The full set of orbital parameters can be found in Table 3. We show in Figure 5 the time series for HD $13167 \mathrm{~b}$ as well as the periodogram before and after subtracting out HD $13167 \mathrm{~b}$. The final observation in the time series (Figure 5) is what secures this detection, as we have now observed it reaching a second maximum.

\subsection{A Jupiter Orbiting HD 18015}

HD 18015 is a G6 subgiant star with $V=7.89$ and $B-V=0.68$ (Wenger et al. 2000). It has a mass of $M_{\star}=$ $1.49 M_{\odot}$, surface gravity $\log g=3.64$, and $T_{\text {eff }}=5603 \mathrm{~K}$. A summary of its stellar parameters can be found in Table 2. Observations of this star span about $8 \mathrm{yr}$ starting in late 2007. The best-fit Keplerian solution yields an orbital period of $2278 \pm 71$ days, and velocity semiamplitude $38.0 \pm$ $2.7 \mathrm{~m} \mathrm{~s}^{-1}$, and eccentricity $0.148 \pm 0.061$. It has a minimum mass of $3.18 \pm 0.23 M_{\text {Jup. }}$ The full set of orbital parameters can be found in Table 3. We show in Figure 6 the time series of HD $18015 \mathrm{~b}$ as well as the periodogram before and after subtracting out HD 18015 b.

\subsection{A Jupiter Orbiting HD 180053}

HD 180053 is a K0 subgiant star with $V=7.93$ and $B-V=0.92$ (Wenger et al. 2000). It has a mass of $M_{\star}=$ $1.75 M_{\odot}$, surface gravity $\log g=3.54$, and $T_{\text {eff }}=5131 \mathrm{~K}$. A summary of its stellar parameters can be found in Table 2. Observations of this star span about $8 \mathrm{yr}$ starting in 2007. HD 180053 was identified in Butler et al. (2017) as having a planet candidate. The best-fit Keplerian solution yields an orbital period of $213.72 \pm 0.47$ days, and velocity semiamplitude $51.5 \pm 1.4 \mathrm{~m} \mathrm{~s}^{-1}$, and eccentricity $0.081 \pm 0.029$. It has a minimum mass of $2.194 \pm 0.063 M_{\text {Jup }}$. The full set of orbital parameters can be found in Table 3. We show in Figure 7 the time series for HD $180053 \mathrm{~b}$ as well as the periodogram before and after subtracting out HD $180053 \mathrm{~b}$. We also include in Figure 8 the phase-folded RV curve to help show the planet signal more clearly. We note that both the phase curve and final periodogram show evidence of additional RV variations beyond simply one planet. Once we remove the best singleplanet fit, we find a correlation between the RVs and the chromospheric activity as measured by the $\mathrm{Ca} \mathrm{II} \mathrm{H}$ and $\mathrm{K}$ lines using the Mount Wilson $s$-index, $S_{\mathrm{HK}}$, measured following the same procedure as in Isaacson \& Fischer (2010). Given that we obtain poor two- and three-planet fits, we expect that the additional RV variations are simply activity-induced, with timescales near 70 and 600 days, reasonable timescales for modulation from stellar rotation and stellar activity cycles.

\subsection{A Jupiter Orbiting HD 4917}

HD 4917 is a K0 subgiant (Wenger et al. 2000). It has a temperature of $T_{\text {eff }}=4802 \mathrm{~K}$ and mass $1.32 M_{\odot}$. Additional stellar properties can be found in Table 2 . There are nearly 50 observations of this star that span close to $10 \mathrm{yr}$. Here we present the discovery of a $m_{\mathrm{p}} \sin i=1.615 \pm 0.093 M_{\text {Jup }}$ planet on a $400.5 \pm 1.7$ day orbit and its phase curve is shown in the left panel of Figure 9. The right panel shows the periodogram before and after subtracting out the best-fit planet.

We note that the final periodogram of the residual shows two intriguing signals: one near 800 days and one near 1000 days. Our best three-planet fit provided the best overall fit; however, we decline to call the outer two real planets for now because we have not done the requisite dynamical analysis to show that the orbits we derive for them are stable. Instead, we consider the outer two signals as planet candidates, which are discussed in Section 4.

\section{Eight Candidate Planetary Signals around Subgiant Stars}

The following signals are likely due to planetary companions. They are intriguing and likely correct; however, they do not rise to the level of the others by not meeting one or more of our detection thresholds, as described in Section 3. We include them here for the purposes of listing possible transit parameters and as long-period planets, as it is uncertain whether more data is forthcoming. We encourage continued observations of these systems to fully confirm these planets. The reason for each planet candidate's status as a candidate is listed individually. 
Table 3

Orbital Parameters

\begin{tabular}{|c|c|c|c|c|c|c|c|c|c|c|c|c|}
\hline Star & $\begin{array}{l}\text { Com } \\
\text { (2) }\end{array}$ & $\begin{array}{c}m \sin i \\
\left(M_{\text {Jup }}\right) \\
(3)\end{array}$ & $\begin{array}{c}R \\
\left(R_{\text {Jup }}\right) \\
(4)\end{array}$ & $\begin{array}{c}P \\
\text { (days) } \\
\text { (5) }\end{array}$ & (6) & $\begin{array}{c}T_{\mathrm{p}} \\
(\mathrm{JD}-2440,000) \\
(7)\end{array}$ & $\begin{array}{l}e \\
\text { (8) }\end{array}$ & $\begin{array}{c}\omega \\
\text { (degrees) } \\
(9)\end{array}$ & $\begin{array}{c}K \\
\left(\mathrm{~m} \mathrm{~s}^{-1}\right) \\
(10)\end{array}$ & $\begin{array}{c}\gamma \\
\left(\mathrm{m} \mathrm{s}^{-1}\right) \\
(11)\end{array}$ & $\begin{array}{l}d v d t \\
\text { (12) }\end{array}$ & $\begin{array}{r}\text { Orbit Ref } \\
\text { (13) }\end{array}$ \\
\hline HD 10697 & b & $6.383(80)$ & 1.152 & $1075.69(82)$ & $2.140(95)$ & $11492(17)$ & $0.1043(83)$ & $114.9(6.0)$ & $116.9(1.3)$ & $-9.15(82)$ & 0 & Wittenmyer et al. (2009) \\
\hline HD 38529 & $\mathrm{~b}$ & $0.797(15)$ & 1.268 & $14.30944(30)$ & $0.1294(58)$ & $14384.59(16)$ & $0.280(17)$ & $89.8(4.4)$ & $55.2(1.1)$ & $8.83(74)$ & 0 & Wright et al. (2009) \\
\hline$\ldots$ & $\mathrm{c}$ & $12.99(15)$ & 1.123 & 2136.1(3.1) & $3.64(16)$ & $12255.9(8.1)$ & $0.3407(69)$ & $18.4(1.4)$ & $172.4(1.3)$ & $0.0(0.0)$ & 0 & Wright et al. (2009) \\
\hline HD 114613 & $\mathrm{~b}$ & $0.357(32)$ & 1.182 & $4000(120)$ & $5.34(26)$ & $15150(90)$ & $0.458(89)$ & 196(11) & $4.38(40)$ & $2.62(65)$ & 0 & Wittenmyer et al. (2014) \\
\hline HD 117176 & $\mathrm{~b}$ & $7.416(54)$ & 1.142 & $116.6880(54)$ & $0.481(21)$ & $15291.721(86)$ & $0.3988(19)$ & $359.83(29)$ & $316.20(78)$ & $-51.25(53)$ & 0 & Butler et al. (2006) \\
\hline HD 159868 & $\mathrm{~b}$ & $2.218(59)$ & 1.202 & $1184.1(7.1)$ & $2.32(12)$ & $13670(190)$ & $0.024(19)$ & $63(76)$ & $37.92(97)$ & $-6.42(92)$ & 0 & Wittenmyer et al. (2012) \\
\hline$\ldots$ & $\mathrm{c}$ & $0.768(44)$ & 1.269 & $351.0(1.1)$ & $1.032(55)$ & $13225(13)$ & $0.184(37)$ & $272(16)$ & $20.0(1.1)$ & $0.0(0.0)$ & 0 & Wittenmyer et al. (2012) \\
\hline HD 175541 & $\mathrm{~b}$ & $0.598(29)$ & 1.277 & $298.43(45)$ & $0.975(89)$ & $10155(25)$ & $0.110(49)$ & $129(28)$ & $14.68(71)$ & $2.06(56)$ & 0 & Johnson et al. (2007a) \\
\hline HD 190228 & $\mathrm{~b}$ & $4.300(59)$ & 1.166 & $1143.5(1.6)$ & $2.24(12)$ & $13521.1(3.5)$ & $0.5571(99)$ & $94.6(1.5)$ & $92.0(1.1)$ & $12.39(77)$ & 0 & Wittenmyer et al. (2009) \\
\hline HD 1502 & $\mathrm{~b}$ & $2.75(16)$ & 1.183 & $428.5(1.2)$ & $1.26(12)$ & $15170(67)$ & $0.031(22)$ & $126(58)$ & $57.5(3.3)$ & $1.32(94)$ & 0 & Johnson et al. (2011) \\
\hline HD 3404 & * & $152.0(6.4)$ & 1.727 & $1543.54(60)$ & $2.75(17)$ & $13438.3(4.9)$ & $0.7440(59)$ & $0.37(21)$ & $3332(17)$ & $116(26)$ & 0 & Binary \\
\hline HD 4313 & $\mathrm{~b}$ & $1.927(90)$ & 1.212 & $356.21(88)$ & $1.157(95)$ & $14816(12)$ & $0.147(47)$ & $102(13)$ & $40.3(1.7)$ & $-13.6(1.7)$ & 0 & Johnson et al. (2010a) \\
\hline HD 5319 & $\mathrm{~b}$ & $1.556(42)$ & 1.227 & $637.1(1.4)$ & $1.57(12)$ & $16382(26)$ & $0.092(25)$ & $158(15)$ & $31.46(83)$ & $-7.21(98)$ & $0.00253(59)$ & Robinson et al. (2007) \\
\hline$\cdots$ & $\mathrm{c}$ & $1.053(72)$ & 1.254 & $872.2(6.0)$ & $1.93(15)$ & $13443(58)$ & $0.183(64)$ & $244(25)$ & 19.4(1.4) & $0.0(0.0)$ & 0 & Giguere et al. (2015) \\
\hline HD 5608 & $\mathrm{~b}$ & $1.681(81)$ & 1.222 & $779.9(4.9)$ & $1.911(92)$ & $12420(110)$ & $0.056(39)$ & $294(54)$ & $28.0(1.4)$ & $39.5(2.8)$ & $-0.0165(12)$ & Sato et al. (2012) \\
\hline HD 6019 & * & $409(36)$ & 4.132 & $2457.00(19)$ & $3.70(32)$ & $21712.74(70)$ & $0.8170(44)$ & $31.18(49)$ & $8120(240)$ & $786(13)$ & 0 & Binary \\
\hline HD 8375 & * & $154.3(4.1)$ & 1.751 & $83.9664(90)$ & $0.441(21)$ & $16221.11(91)$ & $0.0127(10)$ & $319.0(3.9)$ & $4898.7(4.1)$ & $-1506(48)$ & $0.484(21)$ & Binary \\
\hline HD 10011 & * & $609(54)$ & 5.884 & $1518.3(1.2)$ & $2.96(26)$ & $14807.90(59)$ & $0.30677(91)$ & $87.95(19)$ & $6941.7(2.0)$ & $1816.7(1.7)$ & 0 & Binary \\
\hline HD 10212 & * & $664(67)$ & 6.353 & $3353(16)$ & $4.63(41)$ & $15782.5(2.1)$ & $0.5040(30)$ & $104.29(29)$ & $7021.3(9.7)$ & $-1873(12)$ & 0 & Binary \\
\hline HD 10442 & $\mathrm{~b}$ & $1.487(82)$ & 1.230 & $1032.3(8.9)$ & $2.01(54)$ & $17226(69)$ & $0.132(44)$ & $262(25)$ & $29.9(1.6)$ & $-8.0(1.1)$ & 0 & Giguere et al. (2015) \\
\hline HD 11970 & * & $577(58)$ & 5.611 & $8500(140)$ & $8.76(85)$ & $15241.7(1.3)$ & $0.6131(43)$ & $245.56(19)$ & $4933.8(4.2)$ & 1014(12) & 0 & Binary \\
\hline HD 13167 & $\mathrm{~b}$ & $3.31(16)$ & 1.167 & $2613(17)$ & $4.10(43)$ & $22162(54)$ & $0.563(33)$ & $265.0(6.0)$ & $48.2(2.7)$ & $-10.4(1.2)$ & 0 & This Work \\
\hline HD 14787 & $\mathrm{~b}$ & $1.121(69)$ & 1.250 & $676.6(8.1)$ & $1.70(17)$ & $15555(49)$ & $0.155(73)$ & $343(28)$ & $20.7(1.3)$ & $-1.7(1.1)$ & 0 & This Work \\
\hline HD 18015 & $\mathrm{~b}$ & $3.18(23)$ & 1.170 & $2278(71)$ & $3.87(31)$ & $14730(170)$ & $0.148(61)$ & $265(29)$ & $38.0(2.7)$ & $-5.5(1.8)$ & 0 & This Work \\
\hline HD 18667 & * & $309(38)$ & 3.221 & $4376(13)$ & $5.32(73)$ & $15016.83(30)$ & $0.66577(78)$ & $288.968(85)$ & $4218.0(2.2)$ & $-312.6(3.7)$ & 0 & Binary \\
\hline HD 18742 & $\mathrm{~b}$ & $3.4(1.2)$ & 1.166 & $766(25)$ & $1.82(16)$ & $15280(140)$ & $0.040(35)$ & 190(74) & $61(22)$ & $-2.4(3.7)$ & $0.0026(19)$ & Johnson et al. (2011) \\
\hline$\ldots$ & $\mathrm{c}$ & $2.4(1.2)$ & 1.194 & $859(41)$ & $1.96(17)$ & 1910(670) & $0.056(52)$ & 203(63) & $42(22)$ & $0.0(0.0)$ & 0 & This Work \\
\hline HD 21340 & * & $135.9(6.0)$ & 1.566 & $1249.7(2.5)$ & $2.63(20)$ & $15227.4(1.3)$ & $0.5612(90)$ & $129.29(85)$ & $2193(51)$ & $53.4(8.8)$ & 0 & Binary \\
\hline HD 28678 & $\mathrm{~b}$ & $1.542(73)$ & 1.228 & $380.2(1.6)$ & $1.18(18)$ & 15513(18) & $0.149(47)$ & $128(19)$ & $32.9(1.4)$ & $-23.5(3.4)$ & $0.0124(19)$ & Johnson et al. (2011) \\
\hline HD 30856 & $\mathrm{~b}$ & $1.547(91)$ & 1.228 & $847(20)$ & $1.85(13)$ & $15200(150)$ & $0.061(58)$ & $171(68)$ & $29.9(1.7)$ & $5.4(1.7)$ & 0 & Johnson et al. (2011) \\
\hline HD 33142 & $\mathrm{~b}$ & $1.385(64)$ & 1.239 & $326.0(1.2)$ & $1.070(90)$ & $15329(42)$ & $0.066(41)$ & $146(49)$ & $30.7(1.4)$ & $-8.0(1.1)$ & 0 & Johnson et al. (2011) \\
\hline$\ldots$ & $\mathrm{c}$ & $0.62(11)$ & 1.277 & $809(26)$ & $1.96(17)$ & $-150(500)$ & $0.16(15)$ & $126(60)$ & $10.3(1.8)$ & $0.0(0.0)$ & 0 & This Work \\
\hline HD 38801 & $\mathrm{~b}$ & $10.13(24)$ & 1.134 & $686.8(1.4)$ & $1.66(11)$ & $13849(27)$ & $0.059(26)$ & $296(14)$ & $196.3(3.8)$ & $-26.4(2.7)$ & 0 & Harakawa et al. (2010) \\
\hline HD 45410 & $\mathrm{~b}$ & $2.010(77)$ & 1.209 & $934.3(8.6)$ & $2.11(10)$ & $15384(94)$ & $0.073(36)$ & $130(38)$ & $32.8(1.2)$ & $4.4(1.1)$ & 0 & Bowler et al. (2010) \\
\hline HD 51272 & * & $839(97)$ & 7.815 & $5660(680)$ & $7.5(1.2)$ & $15871.5(1.8)$ & $0.669(22)$ & $202.56(61)$ & $6930(130)$ & $3540(360)$ & 0 & Binary \\
\hline HD 72490 & $\mathrm{~b}$ & $1.768(80)$ & 1.218 & $858(12)$ & $1.88(17)$ & $320(230)$ & $0.124(46)$ & $145(23)$ & $33.5(1.5)$ & $-3.4(3.3)$ & $0.0026(20)$ & This Work \\
\hline HD 73534 & $\mathrm{~b}$ & $1.112(59)$ & 1.251 & $1750(24)$ & $2.99(18)$ & $17100(130)$ & $0.126(57)$ & $168(23)$ & 17.11(93) & $-1.67(68)$ & 0 & Valenti et al. (2009) \\
\hline HD 75784 & $\mathrm{~b}$ & $1.00(14)$ & 1.258 & $341.2(1.1)$ & $1.032(68)$ & $41(67)$ & $0.097(63)$ & $350(200)$ & $24.9(3.5)$ & $-30.5(9.5)$ & 0 & Giguere et al. (2015) \\
\hline$\ldots$ & $\mathrm{c}$ & $5.64(72)$ & 1.161 & $7900(2000)$ & $8.4(1.4)$ & $-8800(5900)$ & $0.489(92)$ & $325(12)$ & $56.5(5.2)$ & $0.0(0.0)$ & 0 & Giguere et al. (2015) \\
\hline HD 88133 & $\mathrm{~b}$ & $0.2845(63)$ & 1.045 & $3.414884(30)$ & $0.0479(32)$ & $13016.82(37)$ & $0.031(21)$ & $43(33)$ & $32.93(73)$ & $2.07(53)$ & 0 & Butler et al. (2006) \\
\hline HD 93396 & $\mathrm{~b}$ & $0.201(16)$ & 0.857 & $4.73650(52)$ & $0.0626(50)$ & $17062.26(49)$ & $0.166(91)$ & $115(39)$ & $19.2(1.6)$ & $7.7(4.7)$ & $-0.0019(16)$ & Pepper et al. (2017) \\
\hline HD 94834 & $\mathrm{~b}$ & $1.26(17)$ & 1.242 & $1576(76)$ & $2.74(19)$ & $16100(190)$ & $0.14(10)$ & $38(41)$ & $20.7(2.9)$ & $-2.4(1.5)$ & 0 & This Work \\
\hline HD 95089 & $\mathrm{~b}$ & $3.45(14)$ & 1.165 & $1785(32)$ & $3.33(30)$ & $1170(260)$ & $0.284(42)$ & $66(10)$ & $45.1(1.9)$ & $-15.4(1.3)$ & 0 & Johnson et al. (2010a) \\
\hline$\ldots$ & $\mathrm{c}$ & $1.260(85)$ & 1.242 & $464.4(3.8)$ & $1.36(12)$ & $70(130)$ & $0.119(72)$ & $301(35)$ & $25.0(1.6)$ & $0.0(0.0)$ & 0 & Bryan et al. (2016) \\
\hline HD 96063 & $\mathrm{~b}$ & $1.27(27)$ & 1.242 & $362.5(2.2)$ & $1.11(11)$ & $15165(43)$ & $0.17(11)$ & $271(51)$ & $29.6(6.8)$ & $-14.0(3.9)$ & 0 & Johnson et al. (2011) \\
\hline HD 96167 & $\mathrm{~b}$ & $0.717(40)$ & 1.272 & $498.04(76)$ & $1.332(91)$ & 13061.1(4.4) & $0.685(29)$ & $290.1(6.1)$ & $21.5(1.4)$ & $-0.80(53)$ & 0 & Peek et al. (2009) \\
\hline HD 97601 & * & $367(31)$ & 3.752 & $4140(390)$ & $5.95(62)$ & $16576(12)$ & $0.3365(68)$ & $50.7(4.9)$ & $3120(69)$ & $-460(210)$ & 0 & Binary \\
\hline HD 98219 & $\mathrm{~b}$ & $1.964(99)$ & 1.210 & $433.8(2.0)$ & $1.258(95)$ & $14633(47)$ & $0.079(40)$ & $360(200)$ & $42.0(2.1)$ & $3.5(1.4)$ & 0 & Johnson et al. (2011) \\
\hline HD 99706 & $\mathrm{~b}$ & $1.23(19)$ & 1.244 & $841(32)$ & $1.98(15)$ & $15310(89)$ & $0.25(16)$ & $50(44)$ & $21.2(3.9)$ & $-5.4(6.7)$ & $0.0016(33)$ & Johnson et al. (2011) \\
\hline HD 102956 & $\mathrm{~b}$ & $0.960(23)$ & 1.261 & $6.49470(19)$ & $0.0807(66)$ & $15351.45(64)$ & $0.037(19)$ & $301(33)$ & $74.6(1.8)$ & $-3.0(1.1)$ & 0 & Johnson et al. (2010a) \\
\hline HD 106270 & $\mathrm{~b}$ & $10.13(27)$ & 1.134 & $1888(16)$ & $3.34(21)$ & $16635(37)$ & $0.185(27)$ & $3.3(6.1)$ & $135.4(3.5)$ & $9.8(4.8)$ & $0.0086(27)$ & Johnson et al. (2011) \\
\hline HD 108863 & $\mathrm{~b}$ & $2.414(78)$ & 1.194 & $437.7(2.8)$ & $1.32(11)$ & $15558(77)$ & $0.032(27)$ & 191(64) & $47.4(1.5)$ & $-5.1(1.5)$ & 0 & Johnson et al. (2011) \\
\hline
\end{tabular}


Table 3

(Continued)

\begin{tabular}{|c|c|c|c|c|c|c|c|c|c|c|c|c|}
\hline Star & $\begin{array}{l}\text { Com } \\
\text { (2) }\end{array}$ & $\begin{array}{c}m \sin i \\
\left(M_{\text {Jup }}\right) \\
(3)\end{array}$ & $\begin{array}{c}R \\
\left(R_{\text {Jup }}\right) \\
(4)\end{array}$ & $\begin{array}{c}P \\
\text { (days) } \\
(5)\end{array}$ & $\begin{array}{l}a \\
\text { (6) }\end{array}$ & $\begin{array}{c}T_{\mathrm{p}} \\
(\mathrm{JD}-2440,000) \\
(7)\end{array}$ & (8) & $\begin{array}{c}\omega \\
\text { (degrees) } \\
\text { (9) }\end{array}$ & $\begin{array}{c}K \\
\left(\mathrm{~m} \mathrm{~s}^{-1}\right) \\
(10)\end{array}$ & $\begin{array}{c}\gamma \\
\left(\mathrm{m} \mathrm{s}^{-1}\right) \\
(11)\end{array}$ & $\begin{array}{l}d v d t \\
(12)\end{array}$ & Orbit Ref \\
\hline HD 112988 & ${ }^{*}$ & $346(28)$ & 3.559 & $5780(520)$ & $6.38(71)$ & 16299.0(3.6) & $0.7246(74)$ & $156.7(3.1)$ & $4610(290)$ & $-818(15)$ & 0 & Binary \\
\hline HD 125390 & * & $22.16(96)$ & 1.081 & $1756.2(3.9)$ & $3.16(36)$ & 15914.0(6.7) & $0.591(14)$ & $342.4(1.2)$ & $373(16)$ & $-39.8(3.7)$ & 0 & Binary \\
\hline HD 125607 & * & $379(29)$ & 3.857 & $578.54(13)$ & $1.55(14)$ & $15495.77(54)$ & $0.4084(24)$ & $17.90(41)$ & $6763(31)$ & $-83(10)$ & 0 & Binary \\
\hline HD 131496 & $\mathrm{~b}$ & $1.80(10)$ & 1.217 & $896(16)$ & $2.01(15)$ & 16114(50) & $0.181(60)$ & $58(19)$ & $31.6(1.8)$ & $3.6(1.3)$ & 0 & Johnson et al. (2011) \\
\hline HD 238433 & * & $748(73)$ & 7.060 & $1816.89(26)$ & $3.24(28)$ & $14419.92(43)$ & $0.6106(27)$ & $332.02(24)$ & $9644(87)$ & $886(11)$ & 0 & Binary \\
\hline HD 142091 & $\mathrm{~b}$ & $1.811(57)$ & 1.216 & $1285(14)$ & $2.65(12)$ & $16830(51)$ & $0.167(32)$ & 194(14) & $26.18(86)$ & $-1.74(60)$ & 0 & Baines et al. (2013) \\
\hline HD 145428 & * & $343(28)$ & 3.533 & $5610(350)$ & $6.22(63)$ & $15367.4(5.0)$ & $0.352(25)$ & $309.73(79)$ & $3436(19)$ & $-2129(87)$ & 0 & Binary \\
\hline HD 148284 & * & $34.50(96)$ & 1.067 & $339.302(26)$ & $0.974(79)$ & 15786.93(12) & $0.38967(97)$ & $35.53(15)$ & $1022.7(1.4)$ & $63.21(82)$ & 0 & Binary \\
\hline HD 152581 & $\mathrm{~b}$ & $1.869(68)$ & 1.214 & $686.5(4.8)$ & $1.66(21)$ & $15040(120)$ & $0.040(31)$ & $181(66)$ & $36.2(1.3)$ & $5.21(97)$ & 0 & Johnson et al. (2011) \\
\hline \multirow{3}{*}{$\begin{array}{l}\text { HD } 163607 \\
\ldots \\
\ldots\end{array}$} & $\mathrm{b}$ & $0.7836(98)$ & 1.269 & $75.2203(94)$ & $0.362(16)$ & $14185.93(15)$ & $0.7441(71)$ & $79.6(1.3)$ & $52.34(74)$ & $54(33)$ & 0 & Giguere et al. (2012) \\
\hline & $\mathrm{c}$ & $2.201(37)$ & 1.202 & $1272.0(4.4)$ & $2.39(11)$ & $15111(40)$ & 0.080 & $274(12)$ & $38.37(65)$ & $0.0(0.0)$ & 0 & Giguere et al. (2012) \\
\hline & $\mathrm{d}$ & $7.8(3.7)$ & 1.139 & $37000(13000)$ & $22.4(3.8)$ & $-95000(42000)$ & $0.42(23)$ & $175(92)$ & $49(22)$ & $0.0(0.0)$ & 0 & This Work \\
\hline HD 180053 & $\mathrm{~b}$ & $2.194(63)$ & 1.203 & $213.72(47)$ & $0.843(53)$ & 15197(13) & $0.081(29)$ & $76(21)$ & $51.5(1.4)$ & $2.6(1.1)$ & 0 & This Work \\
\hline \multirow{3}{*}{$\begin{array}{l}\text { HD } 180902 \\
\ldots \\
\ldots\end{array}$} & $\mathrm{b}$ & $1.685(41)$ & 1.221 & $510.9(1.5)$ & $1.40(11)$ & $15055(17)$ & $0.107(22)$ & $181(12)$ & $34.25(84)$ & $259(12)$ & 0 & Johnson et al. (2010a) \\
\hline & * & $98.7(7.6)$ & 1.186 & $5880(440)$ & $7.15(66)$ & $1100(1200)$ & $0.335(25)$ & $73.3(1.6)$ & $898(28)$ & $0.0(0.0)$ & 0 & Binary \\
\hline & c & $0.099(14)$ & 0.564 & $15.9058(55)$ & $0.139(11)$ & $-75.7(5.5)$ & $0.28(13)$ & $67(41)$ & $6.6(1.1)$ & $0.0(0.0)$ & 0 & This Work \\
\hline HD 181342 & $\mathrm{~b}$ & $2.54(19)$ & 1.190 & $564.1(4.1)$ & $1.59(10)$ & $21090(220)$ & $0.022(51)$ & $290(110)$ & $44.1(3.3)$ & $-8.4(2.3)$ & 0 & Johnson et al. (2010a) \\
\hline HD 185269 & $\mathrm{~b}$ & $1.010(14)$ & 1.257 & $6.83776(27)$ & $0.0770(37)$ & $13154.25(11)$ & $0.229(14)$ & $176.2(3.5)$ & $93.3(1.4)$ & $-4.06(86)$ & 0 & Johnson et al. (2006) \\
\hline HD 192699 & $\mathrm{~b}$ & $2.096(93)$ & 1.206 & $340.94(92)$ & $1.063(54)$ & 14079(36) & $0.082(41)$ & $87(37)$ & $49.3(2.1)$ & $22.9(3.3)$ & 0 & Bowler et al. (2010) \\
\hline HD 193342 & * & $380(200)$ & 3.881 & $10000(16000)$ & $10.6(5.6)$ & $15218(82)$ & $0.33(23)$ & $204.5(9.4)$ & $2390(580)$ & $2200(1400)$ & 0 & Binary \\
\hline HD 195787 & * & $531(40)$ & 5.209 & $1909.26(37)$ & $3.37(26)$ & $15589.33(70)$ & $0.2692(43)$ & $84.91(48)$ & $5873(28)$ & $3315(12)$ & 0 & Binary \\
\hline HD 196645 & $\mathrm{~b}$ & $0.497(44)$ & 1.273 & $128.94(41)$ & $0.542(35)$ & 15379(17) & $0.106(91)$ & $89(46)$ & 17.1(1.6) & $2.7(1.1)$ & 0 & This Work \\
\hline \multirow[t]{2}{*}{ HD 200964} & $\mathrm{~b}$ & $1.599(67)$ & 1.225 & $606.3(3.8)$ & $1.565(79)$ & $15350(49)$ & $0.087(35)$ & $133(30)$ & $30.9(1.3)$ & $-6.16(72)$ & 0 & Johnson et al. (2011) \\
\hline & $\mathrm{c}$ & $1.214(72)$ & 1.244 & $852.5(7.1)$ & $1.96(10)$ & 15044(29) & $0.243(47)$ & $301(13)$ & $21.5(1.3)$ & $0.0(0.0)$ & 0 & Johnson et al. (2011) \\
\hline HD 206610 & $\mathrm{~b}$ & $2.036(62)$ & 1.208 & $673.2(3.3)$ & $1.74(23)$ & 14724(34) & $0.100(42)$ & $334(16)$ & $35.4(1.0)$ & $29.8(2.5)$ & $-0.0242(15)$ & Johnson et al. (2010a) \\
\hline HD 207077 & $\mathrm{~b}$ & $1.16(10)$ & 1.248 & $606.3(5.6)$ & $1.46(14)$ & $15967(40)$ & $0.204(99)$ & $248(26)$ & $26.2(2.5)$ & $-10.3(1.5)$ & 0 & This Work \\
\hline HD 210702 & $\mathrm{~b}$ & $1.808(97)$ & 1.216 & $354.10(70)$ & $1.148(55)$ & $14042(64)$ & $0.028(34)$ & $189(66)$ & $37.8(2.0)$ & $15.7(1.5)$ & 0 & Bowler et al. (2010) \\
\hline HD 212771 & $\mathrm{~b}$ & $2.39(27)$ & 1.195 & $380.7(1.4)$ & $1.192(99)$ & $14920(52)$ & $0.076(51)$ & $29(65)$ & $50.0(5.8)$ & 14.1(3.3) & 0 & Johnson et al. (2010a) \\
\hline HD 214823 & * & $20.56(32)$ & 1.082 & 1853.9(1.6) & $3.23(20)$ & $15642.1(8.6)$ & $0.1633(36)$ & $122.9(1.7)$ & $285.2(1.3)$ & $-13.48(90)$ & 0 & Binary \\
\hline HD 4917 & b & $1.615(93)$ & 1.224 & $400.5(1.7)$ & $1.17(10)$ & $-212(84)$ & $0.066(41)$ & 313(34) & $37.1(2.2)$ & $5.7(1.7)$ & 0 & This Work \\
\hline$\cdots$ & c & $1.37(13)$ & 1.236 & $821(13)$ & $1.88(17)$ & $1000(230)$ & $0.467(88)$ & $45(11)$ & $27.9(3.7)$ & $0.0(0.0)$ & 0 & This Work \\
\hline$\ldots$ & $\mathrm{d}$ & $0.89(10)$ & 1.264 & 1093(37) & $2.28(21)$ & $450(530)$ & $0.28(14)$ & $296(27)$ & $15.2(2.2)$ & $0.0(0.0)$ & 0 & This Work \\
\hline
\end{tabular}

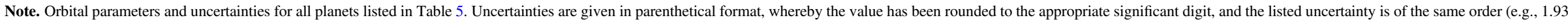

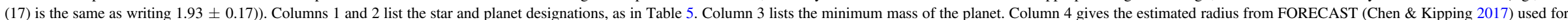

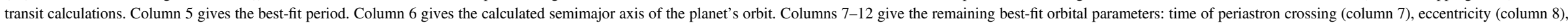

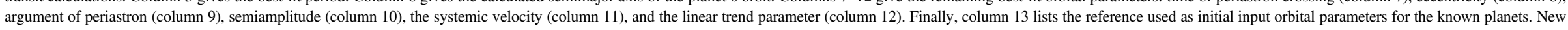

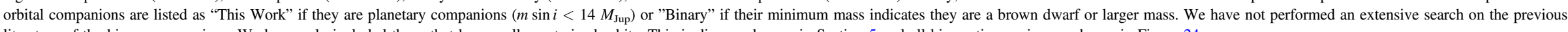
literature of the binary companions. We have only included those that have well-constrained orbits. This is discussed more in Section 5 and all binary time series are shown in Figure 24 .

(This table is available in machine-readable form.) 

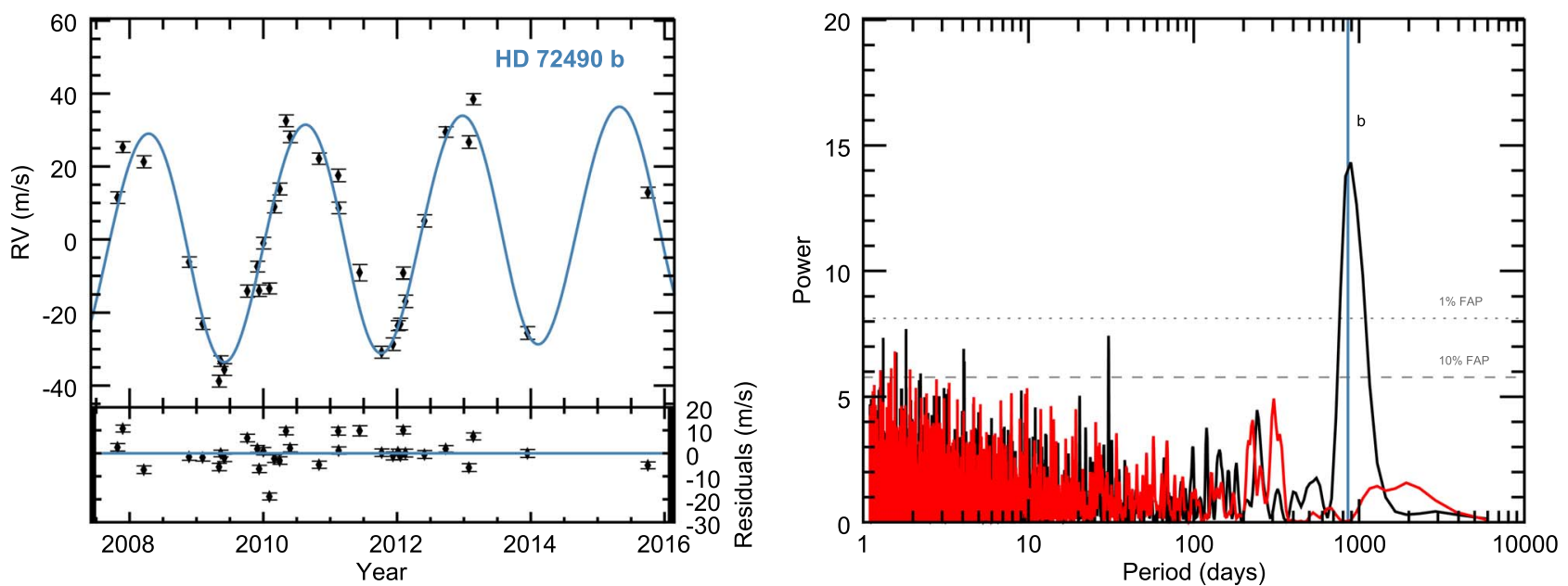

Figure 2. (Left) Time series and best-fit orbital solution for HD $72490 \mathrm{~b}$, which has period $P=858.67$ days, eccentricity $e=0.0569$, and minimum mass $m_{\mathrm{p}} \sin i=1.709 M_{\mathrm{Jup}}$. The residuals are shown in the bottom panel, which have an RV rms $=6.43 \mathrm{~m} \mathrm{~s}^{-1}$. The remaining best-fit parameters can be found in Table 3 . (Right) Periodogram of HD 72490 RV data before (black) and after (red) subtracting the best-fit planet parameters for HD 72490 b. The vertical line indicates the bestfit period of HD $72490 \mathrm{~b}$.
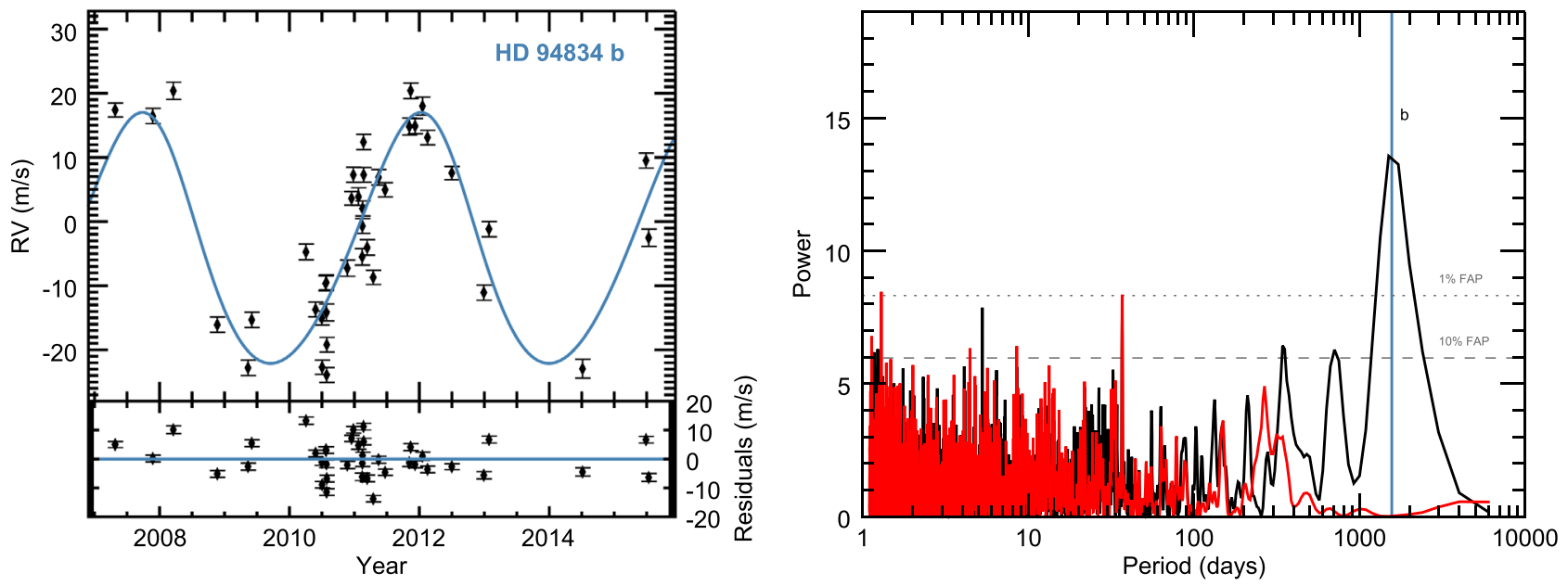

Figure 3. (Left) Time series and best-fit orbital solution for HD $94834 \mathrm{~b}$, which has period $P=1576$ days, eccentricity $e=0.107$, and minimum mass $m_{\mathrm{p}} \sin i=1.2$ $M_{\text {Jup. }}$. The residuals are shown in the bottom panel, which have an RV rms $=6.32 \mathrm{~m} \mathrm{~s}^{-1}$. The remaining best-fit parameters and uncertainties can be found in Table 3 . (Right) Periodogram of HD 94834 RV data before (black) and after (red) subtracting the best-fit planet parameters for HD 94834 b. The vertical line indicates the bestfit period of HD $94834 \mathrm{~b}$
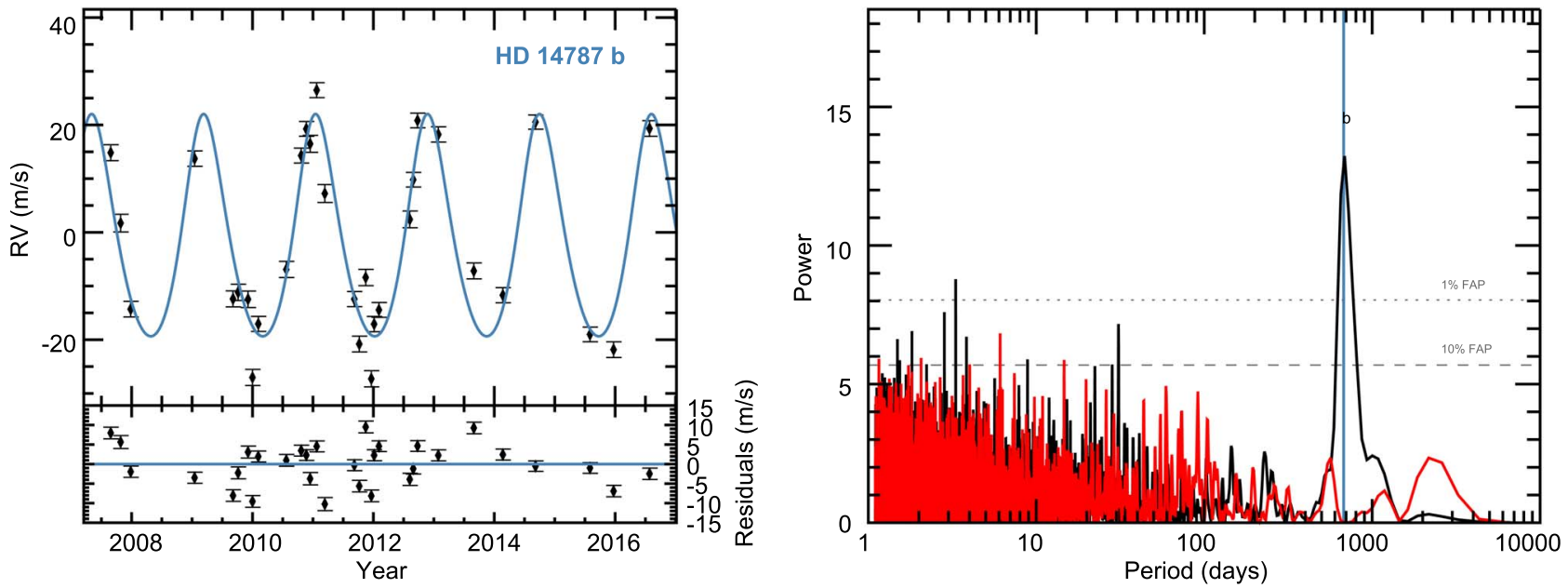

Figure 4. (Left) Time series and best-fit orbital solution for HD $14787 \mathrm{~b}$, which has period $P=676$ days, eccentricity $e=0.155$, and minimum mass $m_{\mathrm{p}}$ sin $i=1.12$ $M_{\text {Jup. }}$. The residuals are shown in the bottom panel, which have an RV rms $=5.3 \mathrm{~m} \mathrm{~s}^{-1}$. The remaining best-fit parameters can be found in Table 3. (Right) Periodogram of HD $14787 \mathrm{RV}$ data before (black) and after (red) subtracting the best-fit planet parameters for HD $14787 \mathrm{~b}$. The vertical line indicates the best-fit period of HD $14787 \mathrm{~b}$. 

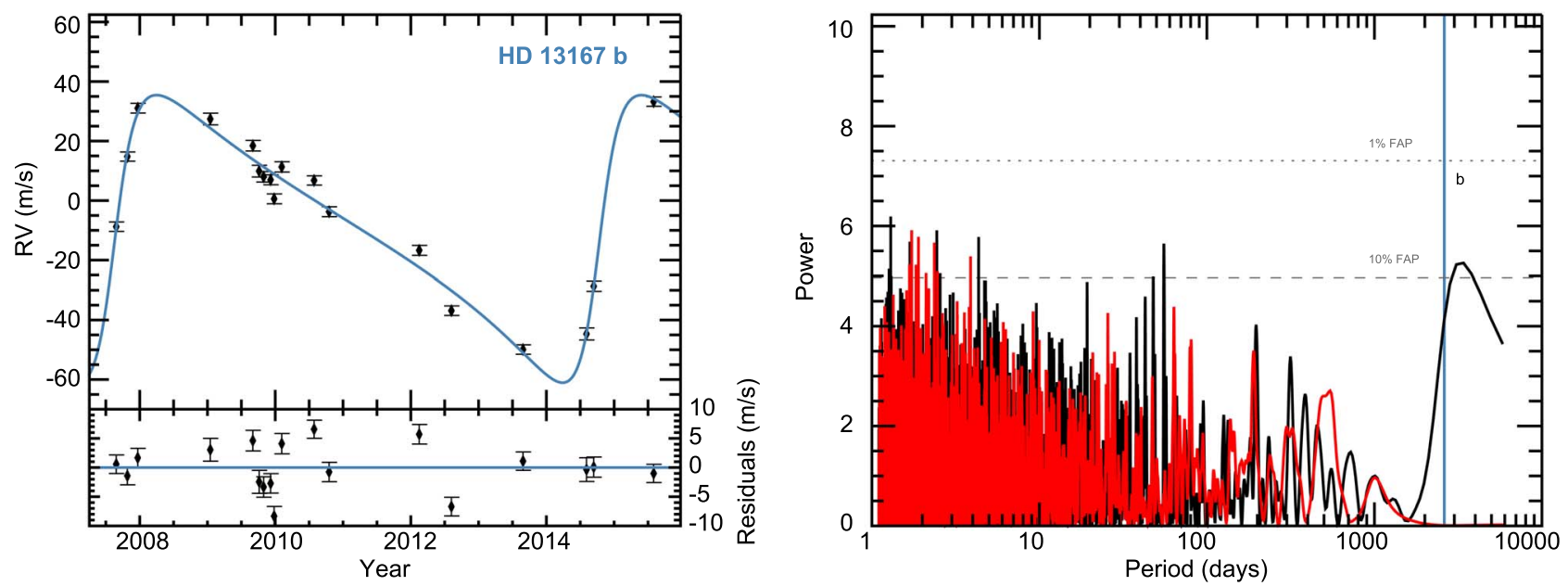

Figure 5. (Left) Time series and best-fit orbital solution for HD $13167 \mathrm{~b}$, with period $P=2613$ days, eccentricity $e=0.563$, and minimum mass $m_{\mathrm{p}}$ sin $i=3.31$ $M_{\text {Jup }}$. The residuals are shown in the bottom panel, which have an $\mathrm{RV} \mathrm{rms}=4.0 \mathrm{~m} \mathrm{~s}^{-1}$. The remaining best-fit parameters can be found in Table 3 . (Right) Periodogram of HD 13167 RV data before (black) and after (red) subtracting the best-fit planet parameters for HD $13167 \mathrm{~b}$. The vertical line indicates the best-fit period of HD $13167 \mathrm{~b}$. The peak in the periodogram is only barely visible because the time baseline of observations is only slightly longer than 1 period.
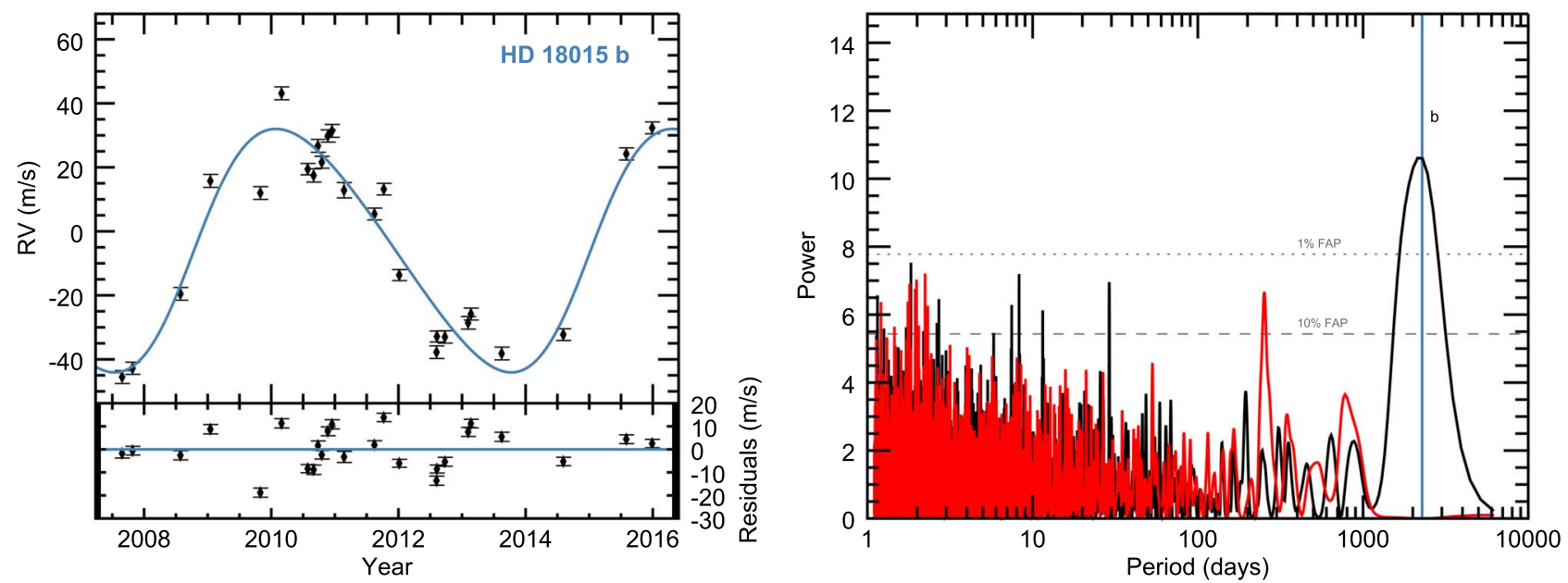

Figure 6. (Left) Time series and best-fit orbital solution for HD $18015 \mathrm{~b}$, which has period $P=2278$ days, eccentricity $e=0.148$, and minimum mass $m_{\mathrm{p}} \sin i=3.18 M_{\mathrm{Jup}}$. The residuals are shown in the bottom panel, which have an RV rms $=8.4 \mathrm{~m} \mathrm{~s}^{-1}$. The remaining best-fit parameters can be found in Table 3 . (Right) Periodogram of HD 18015 RV data before (black) and after (red) subtracting the best-fit planet parameters for HD $18015 \mathrm{~b}$. The vertical line indicates the bestfit period of HD $18015 \mathrm{~b}$.

\subsection{Two Possible Additional Planets around HD 4917}

The two additional signals around HD 4917, planets c and d, described above are both convincing signals. HD $4917 \mathrm{c}$ has an orbital period $P=821 \pm 13$ days, eccentricity $e=0.467 \pm$ 0.088 , and minimum mass $m_{\mathrm{p}} \sin i=1.37 \pm 0.13 M_{\mathrm{Jup}}$ and HD $4917 \mathrm{~d}$ has orbital period $P=1093 \pm 37$ days, eccentricity $e=0.28 \pm 0.14$, and minimum mass $m_{\mathrm{p}} \sin i=0.89 \pm 0.1$

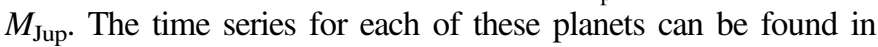
Figure 10 as well as a periodogram before and after subtracting out these two additional signals (HD 4917b was discussed in Section 3). The full time series including the three-planet fit is shown in Figure 11. However, given the proximity of these two additional signals, we feel that this system warrants additional dynamical investigations to ascertain the stability of this planetary system. That detailed investigation is beyond the scope of this work and so we consider these two signals as planetary candidates, despite passing our planet thresholds.

\subsection{A Possible Second Planet around HD 18742}

HD 18742 is a G8/K0 IV star (Wenger et al. 2000) with one previously known planet (Johnson et al. 2011). It has a $V$ magnitude $V=7.81$, effective temperature $T_{\text {eff }}=4940 \mathrm{~K}$, and $\log g=3.09$. Its mass is $1.36 M_{\odot}$ and it has a radius of $5.13 R_{\odot}$. Additional stellar properties and uncertainties can be found in Table 2. There are $37 \mathrm{RV}$ observations for this star, all from Keck, which span more than 8 yr (2007 to late 2015). Here we make use of the additional 11 points that span the last $4.5 \mathrm{yr}$ of the $8 \mathrm{yr}$ of observations. The original fit for this planet in Johnson et al. (2011) included a linear trend. Indeed, after refitting this planet and subtracting out the new best fit to HD $18742 \mathrm{~b}$ (period $P=766 \pm 25$ days, eccentricity $e=$ $0.040 \pm 0.035$, and minimum mass $m_{\mathrm{p}} \sin i=3.4 \pm 1.2 M_{\mathrm{Jup}}$ ), there was a substantial peak in the periodogram near 900 days, shown in Figure 12. Refitting the RVs with a two-planet fit starting with a second planet near 900 days resulted in an 

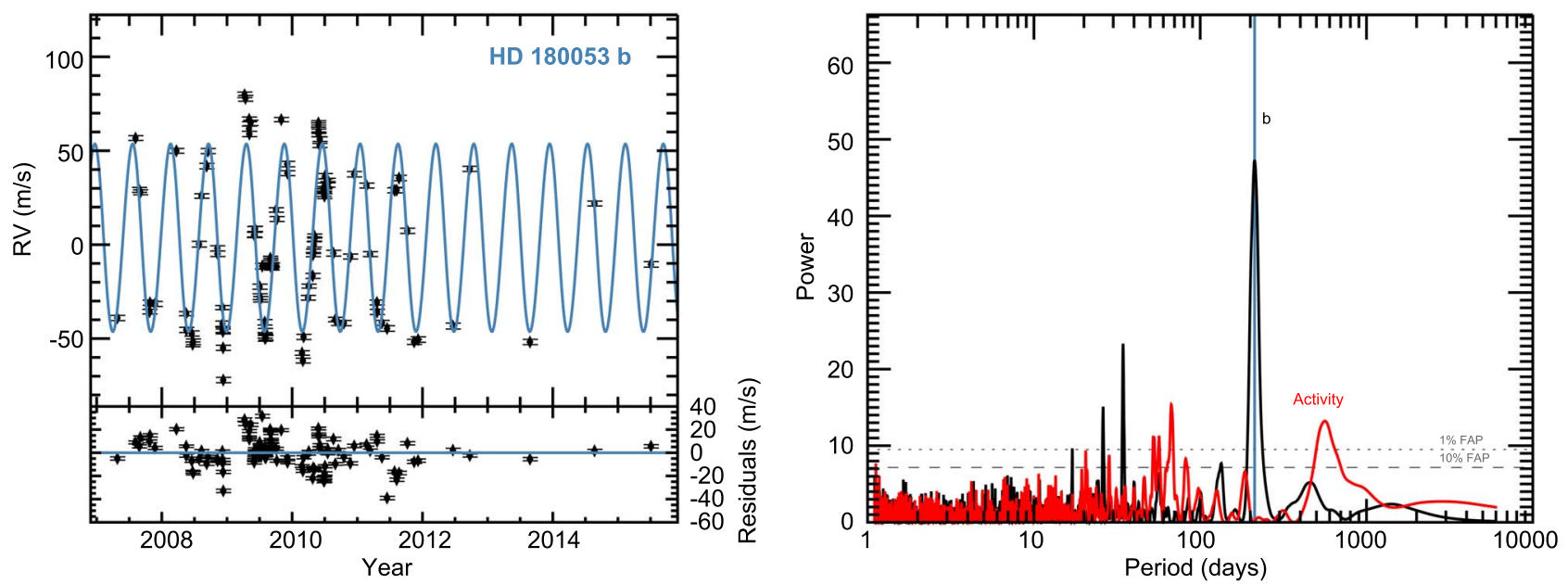

Figure 7. (Left) Time series of the best-fit orbital solution for HD $180053 \mathrm{~b}$, which has period $P=213.72$ days, eccentricity $e=0.081$, and minimum mass $m_{\mathrm{p}} \sin i=2.194 M_{\mathrm{Jup}}$. The residuals are shown in the bottom panel, which have an RV rms $=13.8 \mathrm{~m} \mathrm{~s}^{-1}$, likely due to intrinsic variability induced by stellar activity. The remaining best-fit parameters can be found in Table 3. (Right) Periodogram of HD 180053 RV data before (black) and after (red) subtracting the best-fit planet parameters for HD $180053 \mathrm{~b}$. The vertical line indicates the best-fit period of HD $180053 \mathrm{~b}$. The remaining peaks in the periodogram are likely due to RV variations induced by stellar activity.

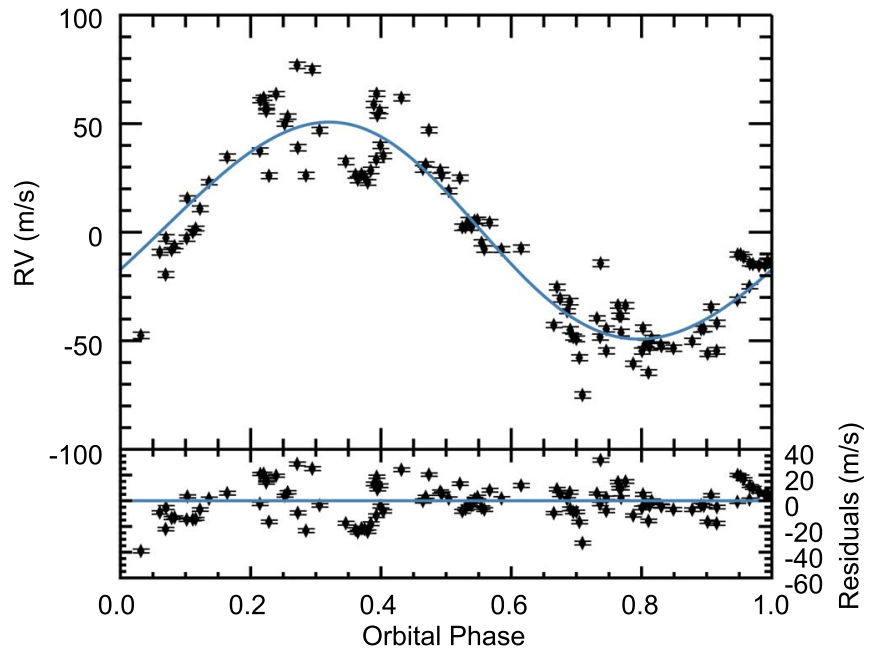

Figure 8. Phase-folded velocities of HD 180053 b.

improvement in the reduced $\chi^{2}$ from 78 to 20 and finds HD 18742 c to have a period of $859 \pm 41$ days, eccentricity $e=$ $0.056 \pm 52$, and minimum mass $m_{\mathrm{p}} \sin i=2.4 \pm 1.2 M_{\text {Jup. }}$. See Table 3 for more orbital and planet parameters and uncertainties. The time series for the second planet (HD 18742c) is shown in the left panel of Figure 12 with the full time series and twoplanet fit shown in Figure 13.

Despite providing a much better fit and meeting all of our planet detection thresholds, we have listed it as a candidate signal because the resulting planet would be in a 9:10 resonance with the inner planet, which is nonphysical and would be the first set of planets in such a resonance. As a result, further scrutiny is needed in order to confirm or reject this planet. Given that the location of the periodogram peak is closer to 1000 days than 860 days (see Figure 12), it could simply be that we have found a shallow minimum in the twoplanet fit that results in the unphysical resonance. Additional observations should uncover the true nature of this signal. Particularly, the one-planet and two-planet models reach their largest divergence in mid-2018 (differ by $60 \mathrm{~m} \mathrm{~s}^{-1}$ ) and mid2019 (differ by nearly $90 \mathrm{~m} \mathrm{~s}^{-1}$ ). Past observations mainly lie in regions where the two solutions differ by $10-20 \mathrm{~m} \mathrm{~s}^{-1}$ with a few points out to $30-40 \mathrm{~m} \mathrm{~s}^{-1}$ (all points more closely tracing the two-planet solution).

\subsection{A Possible Third Planet around HD 163607}

HD 163607 is a G5 IV star (Wenger et al. 2000) with two previously known planets (Giguere et al. 2012). It has a $V$ magnitude $V=7.979$, effective temperature $T_{\text {eff }}=5522 \mathrm{~K}$, and $\log g=3.97$. It is a $1.12 M_{\odot}$ star with radius $1.76 R_{\odot}$. For additional stellar parameters and uncertainties, see Table 2. There are $73 \mathrm{RV}$ observations of this star, the final 20 of which are additional points since the original discovery of HD $163607 \mathrm{~b}$ and c. All $73 \mathrm{RV}$ observations come from Keck. After refitting the two planets and subtracting out the new best fits, there was a strong long-period signal in the periodogram, shown in the right panel of Figure 14. Refitting the time series with the inclusion of a third, long-period planet resulted in an improvement in the reduced $\chi^{2}$ from 37 to 8 . The time series for HD 163607 d is shown in Figure 14 with the full time series for all three planets shown in Figure 15. As is obvious from the time series, we have not covered a full orbit for this planet, which is why it is a planet candidate. We note that due to the long period of the planet and incomplete phase coverage, the derived orbital parameters for planet $\mathrm{d}$ are fairly uncertain, which will be resolved with future observations. In particular, the addition of the most recent observation (2018 September) has shown that the outer planet is indeed very long period and massive. Future observations will continue to constrain the outer planet, which in the current best fit has a mass $7.6 \pm 4.3 M_{\text {Jup }}$, period 22,000 $\pm 15,000$ days, and eccentricity $0.25 \pm 0.25$. Additional orbital parameters can be found in Table 3. If confirmed, this (or similarly HD 4917 above) will be the first three-planet system around a subgiant, which could be useful in determining evolutionary properties of planets around subgiant stars. The novelty of the three-planet subgiant system and probability of transit warrants its inclusion here despite lacking a fully constrained orbit. 

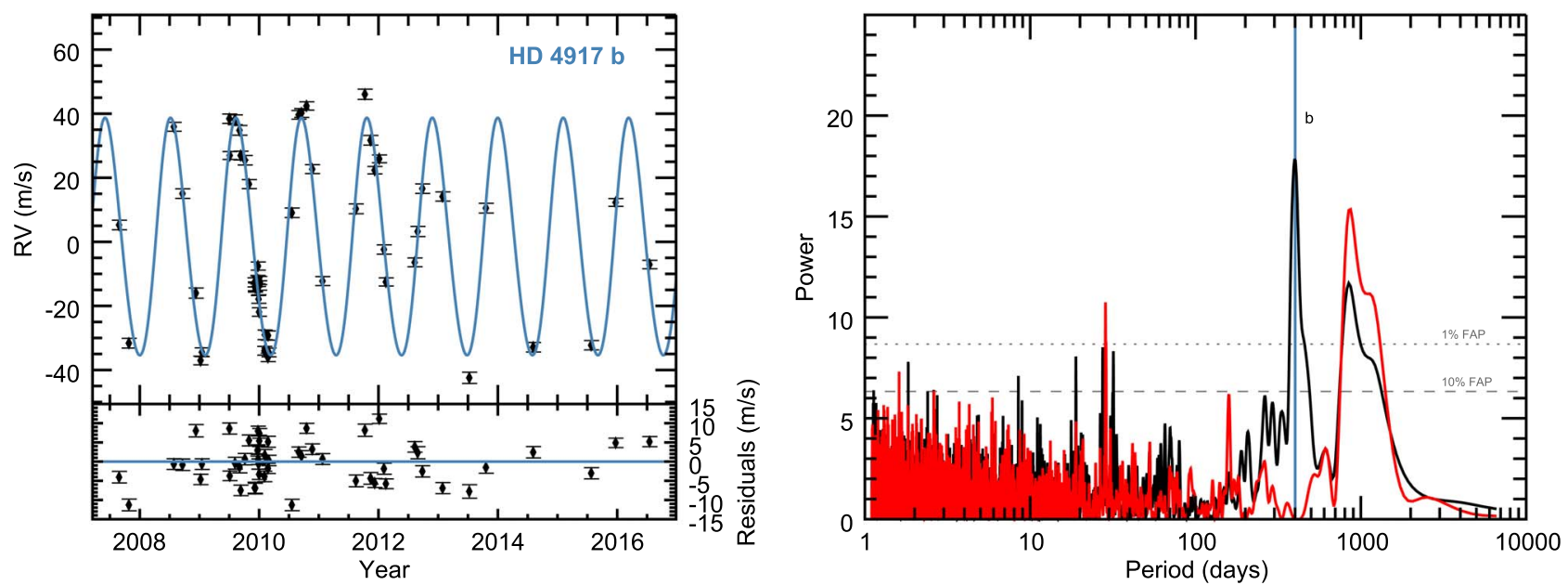

Figure 9. (Left) The isolated time series of HD $4917 \mathrm{~b}$ (period $P=400.5 \pm 1.7$ days, eccentricity $e=0.066 \pm 0.041$, and minimum mass $m_{\mathrm{p}} \sin i=1.615 \pm 0.093$ $M_{\text {Jup }}$ ). In this panel the signal from planet candidates c (period $P=821 \pm 13$ days, eccentricity $e=0.467 \pm 0.088$, and minimum mass $m_{\mathrm{p}} \sin i=1.37 \pm 0.13$ $M_{\mathrm{Jup}}$ ) and d (period $P=1093 \pm 37$ days, eccentricity $e=0.28 \pm 0.14$, and minimum mass $m_{\mathrm{p}} \sin i=0.89 \pm 0.1 M_{\text {Jup }}$ ) have been subtracted out. (Right) Initial (black) and final (red) periodogram of HD 4917 after subtracting out the signal from planet b. The final periodogram shows the two peaks corresponding to planet candidates $\mathrm{c}$ and $\mathrm{d}$.
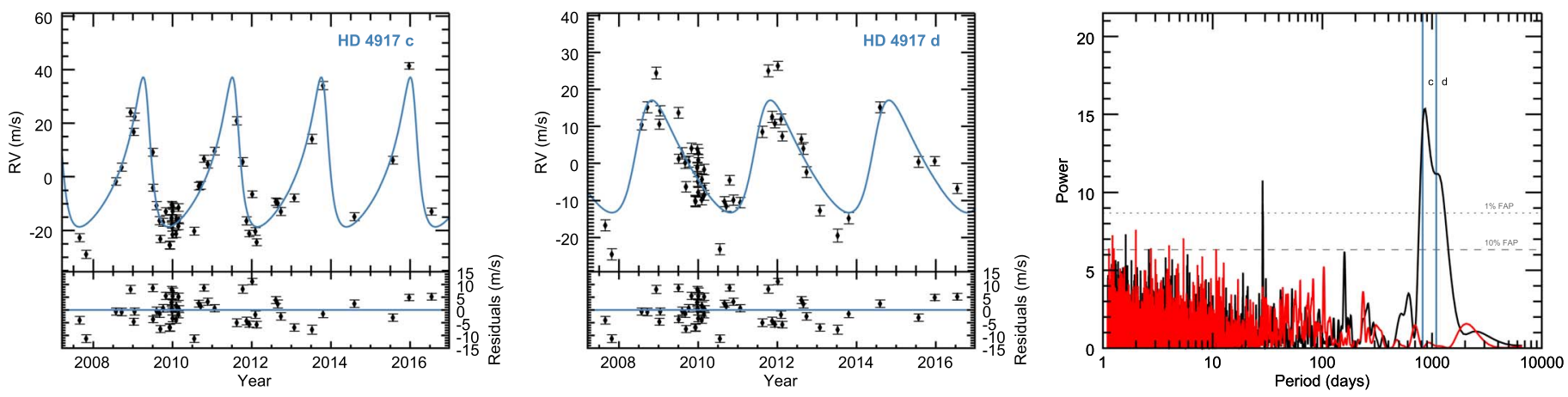

Figure 10. Two additional planet candidates around HD 4917. (Left) The isolated time series of HD $4917 \mathrm{c}$ (period $P=821 \pm 13$ days, eccentricity $e=0.467 \pm 0.088$, and minimum mass $m_{\mathrm{p}} \sin i=1.37 \pm 0.13 M_{\mathrm{Jup}}$ ). In this panel, the signals from planet $\mathrm{b}$ (period $P=400.5 \pm 1.7$ days, eccentricity $e=0.066 \pm 0.041$, and minimum mass $m_{\mathrm{p}} \sin i=1.615 \pm 0.093 M_{\mathrm{Jup}}$ ) and planet candidate $\mathrm{d}$ (period $P=1093 \pm 37$ days, eccentricity $e=0.28 \pm 0.14$, and minimum mass $m_{\mathrm{p}} \sin i=0.89 \pm 0.1 M_{\mathrm{Jup}}$ ) have been subtracted out. (Middle) The isolated time series of HD $4917 \mathrm{~d}$ with signals from planet b and planet candidate c subtracted out. (Right) Periodogram before (black) and after (red) subtracting out the two additional planet candidates HD $4917 \mathrm{c}$ and d. In this case, the initial periodogram is after subtracting out planet $b$, and is identical to the red periodogram in Figure 9.

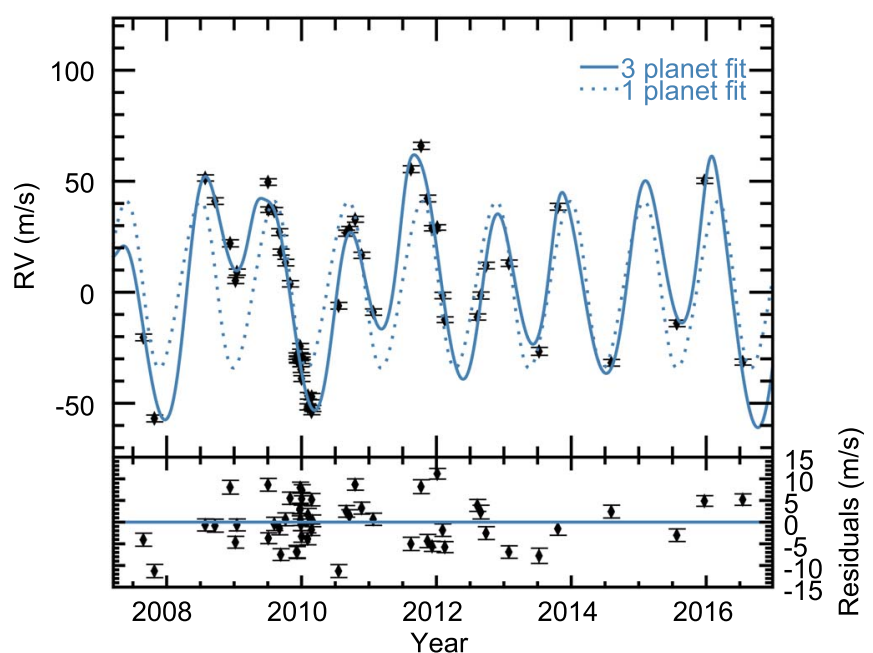

Figure 11. Full time series for HD 4917 and best three-planet fit compared to the best one-planet fit. A single planet does not fit well, especially evidenced by the observations between mid-2008 and mid-2010. The bottom panel shows the residuals to the three-planet fit.

\subsection{A Possible Short-period Planet Orbiting HD 180902}

HD 180902 is a K0 star (Wenger et al. 2000) with one previously known planet (Johnson et al. 2010b). Its $V$-band magnitude is $V=7.78$, its effective temperature is $T_{\text {eff }}=$ $4961 \mathrm{~K}$, and it has a surface gravity $\log g=3.36$. It has a mass of $1.41 M_{\odot}$ and radius $4.16 R_{\odot}$. The full list of stellar parameters and uncertainties is given in Table 2. There are 28 total RV observations for HD 180902, the final 17 of which are new observations that span over $6 \mathrm{yr}$ since the original discovery of HD 180902 (Johnson et al. 2010b). It was noted in Johnson et al. (2010b) and later Bryan et al. (2016) that there was a long-term linear trend that was subtracted out, which was speculated to be a long-period companion. With the additional observations available since its discovery, we are now able to obtain a Keplerian signal to this trend. The trend is indeed due to a companion, which we estimate to be a low-mass star with minimum mass $98.7 \pm 7.6 M_{\text {Jup }}$. However, after performing this fit, an additional 15 day signal appeared in the periodogram. Fitting the system with three companions resulted in the best fit (bringing the reduced $\chi^{2}$ from 12 in the two-companion 

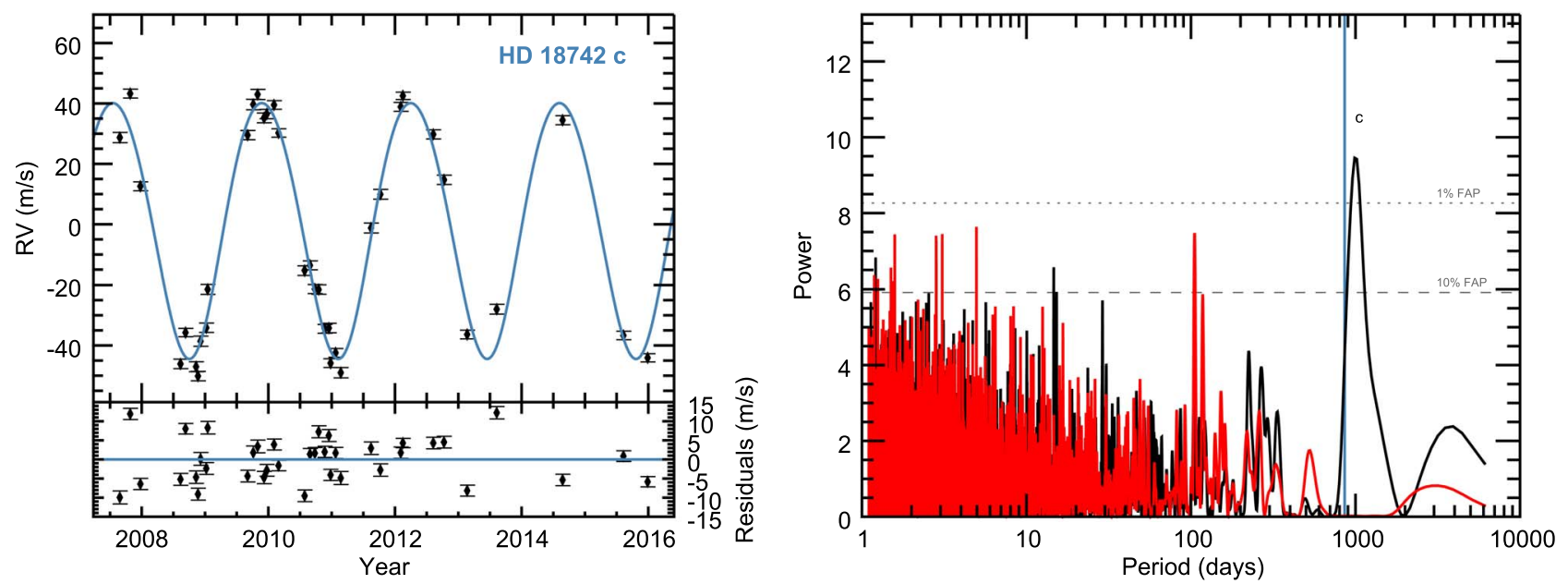

Figure 12. Residuals after subtracting out the previously discovered planet HD $18742 \mathrm{~b}$ (period $P=766$ days, eccentricity $e=0.040$, and minimum mass $m_{\mathrm{p}} \sin i=3.4 M_{\text {Jup }}$ ), showing evidence of a second planet. (Left) Time series for HD $18742 \mathrm{c}$ with period 859 days, eccentricity $e=0.056$, and minimum mass $m_{\mathrm{p}} \sin i=2.4 M_{\text {Jup. }}$. (Right) Periodogram after subtracting out the best-fit orbital parameters for HD $18742 \mathrm{~b}$ (black) and after subtracting out the best two-planet fit (black). Note the peak in the black curve at about 900 days, the starting guess used in the two-planet fit. The vertical line of the best-fit planet period does not quite match the periodogram peak. This is likely due to the fact that the vertical line comes from the best-fit period of a two-planet joint fit, rather than simply a fit to the residuals of the first planet, which is shown in the periodogram. We note that the best two-planet fit results in an unphysical 9:10 resonance, which is why we consider HD 18742 c a planet candidate.

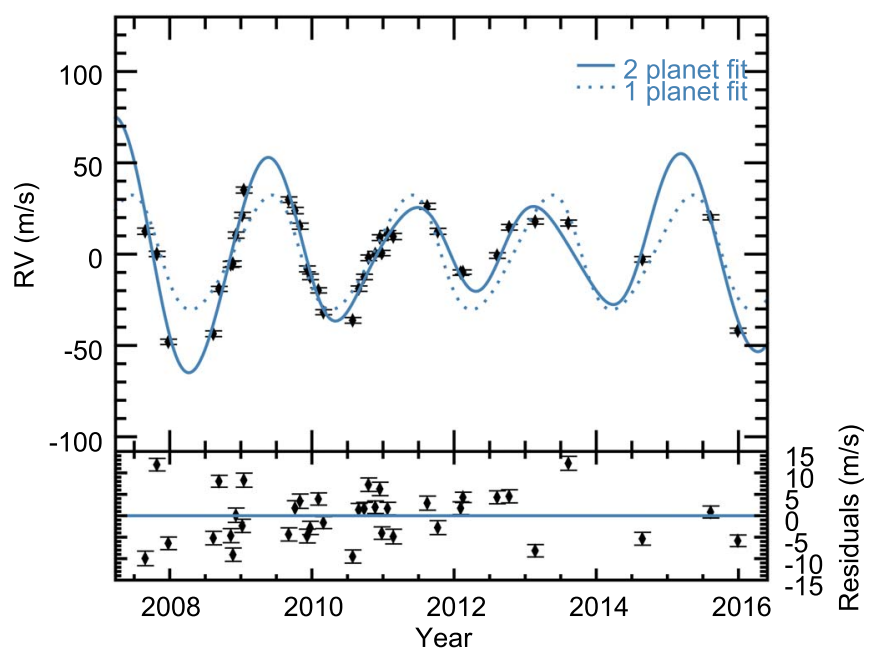

Figure 13. Full time series for HD 18742 and best two-planet fit. The dotted line shows for reference the best one-planet fit. The bottom panel shows the residuals to the two-planet fit. We note that the best two-planet fit results in an unphysical 9:10 resonance, which is why we consider HD $18742 \mathrm{c}$ a planet candidate.

fit to 4 in the three-companion fit). With this fit, the system is therefore composed of two planets (HD $180902 \mathrm{~A} \mathrm{~b}, \mathrm{c}$ ), with a low-mass stellar companion (HD $180902 \mathrm{~B}$ ). Like the longperiod planet HD $163607 \mathrm{c}$, the phase coverage for stellar companion HD $180902 \mathrm{~B}$ is incomplete and so period and mass uncertainties are quite high. We expect this to be resolved as more observations are obtained. The periodogram showing the peak at 15 days for HD $180902 \mathrm{c}$ is shown in the right panel of Figure 16, with the time series shown in the left panel. The phase curve for HD $180902 \mathrm{c}$ is shown in Figure 17. HD $180902 \mathrm{c}$ has a minimum mass roughly twice the mass of Neptune, at $0.099 \pm 0.014 M_{\text {Jup }}$. Finally, the best-fit Keplerian signal for HD 180902 B is given in Figure 18, which shows the signal from HD $180902 \mathrm{~B}$ with the RV signals from HD $180902 \mathrm{~b}$ and $\mathrm{c}$ subtracted out. More phase coverage is needed to place tighter constraints on the orbit of this companion. Orbital parameters for both stellar companion HD 180902 B and planet HD $180902 \mathrm{c}$ are given in Table 3.

We have identified this planet as a planet candidate due to its high FAP, $1.2 \%$. With the current observations, we are not fully convinced by the 15 day planet: the periodogram does not show a very strong signal, and the phase curve is sparsely sampled. Additionally, a 15 day period raises concerns of stellar rotation timescales and potentially activity-induced RV variations. Additionally, with a poorly constrained stellar binary, it is possible that this 15 day signal would be resolved simply with a more accurate fit to the stellar companion. With more observations, we should be able to confirm the existence of this planet. We further note that it has a relatively high transit probability. A detected transit, either ground-based or from TESS, would be able to confirm this planet, which is why we have included this candidate signal.

\subsection{A Possible Sub-Jupiter Orbiting HD 196645}

HD 196645 is a K0 subgiant with $V=7.80, B-V=0.91$ (Wenger et al. 2000). It has effective temperature $T_{\text {eff }}=$ $5041 \mathrm{~K}$, and surface gravity $\log g=3.43$. A summary of its stellar parameters can be found in Table 2 .

It has 20 observations that span nearly $6 \mathrm{yr}$. The best-fit orbital solution yields a $m_{\mathrm{p}} \sin i=0.497 \pm 44 M_{\text {Jup }}$ planet on a $128.94 \pm 0.41$ day period, with relatively low eccentricity, $e=0.106 \pm 0.091$. The full set of orbital parameters are given in Table 3. Figure 19 shows the time series of HD 196645 b, with the initial and final periodogram for this system. For clarity, Figure 20 also shows the phase-folded velocities for HD 196645 b. For this star, the low semiamplitude and few observations keep us from definitively claiming this planet. It has FAP of $1.0 \%$ and is close to our $D>10$ threshold at $D \sim 13.6$, which in combination lead to its candidate status. Continued observations will likely add significance to the periodogram peak and result in better phase coverage. 

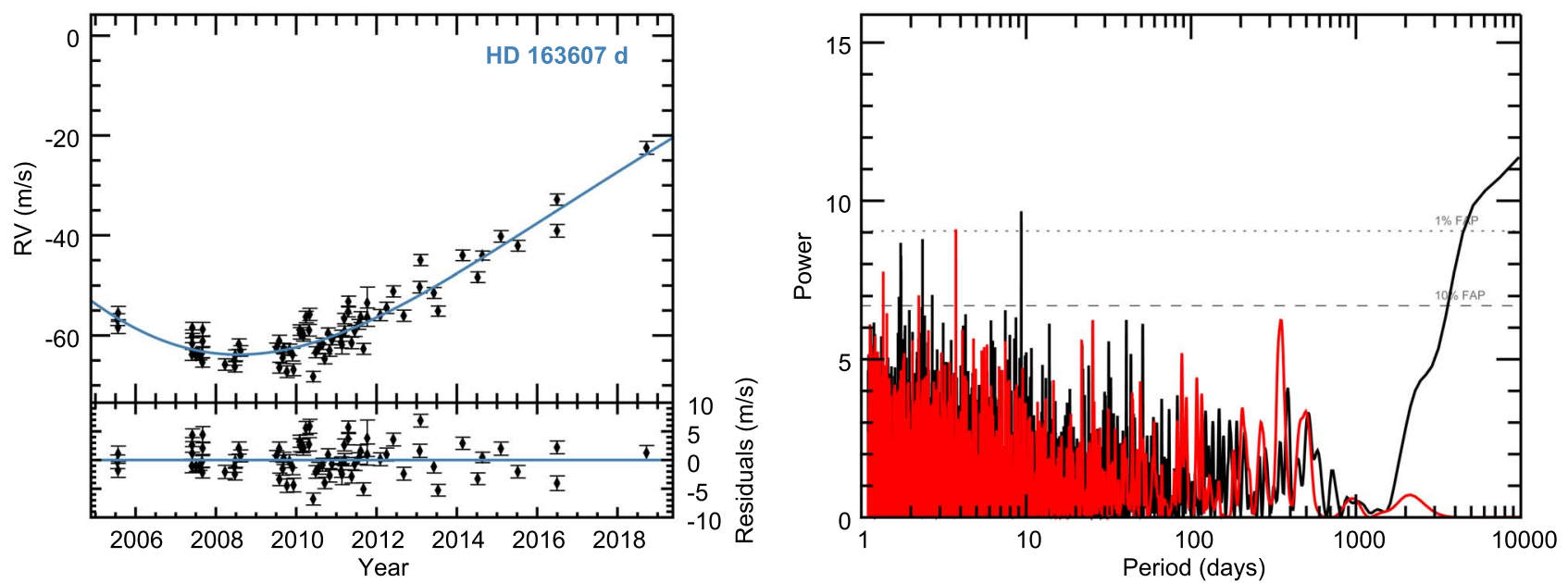

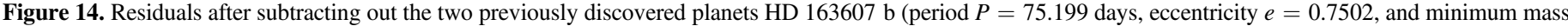

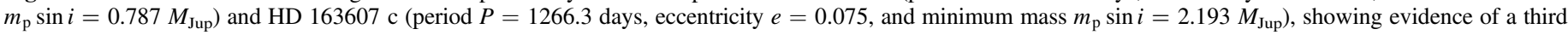

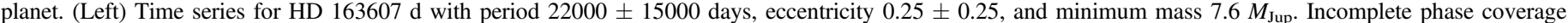

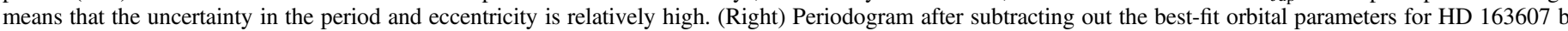

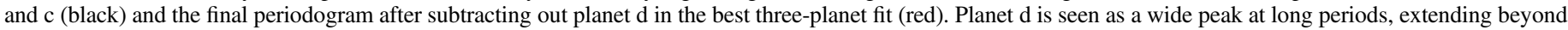
the plot.

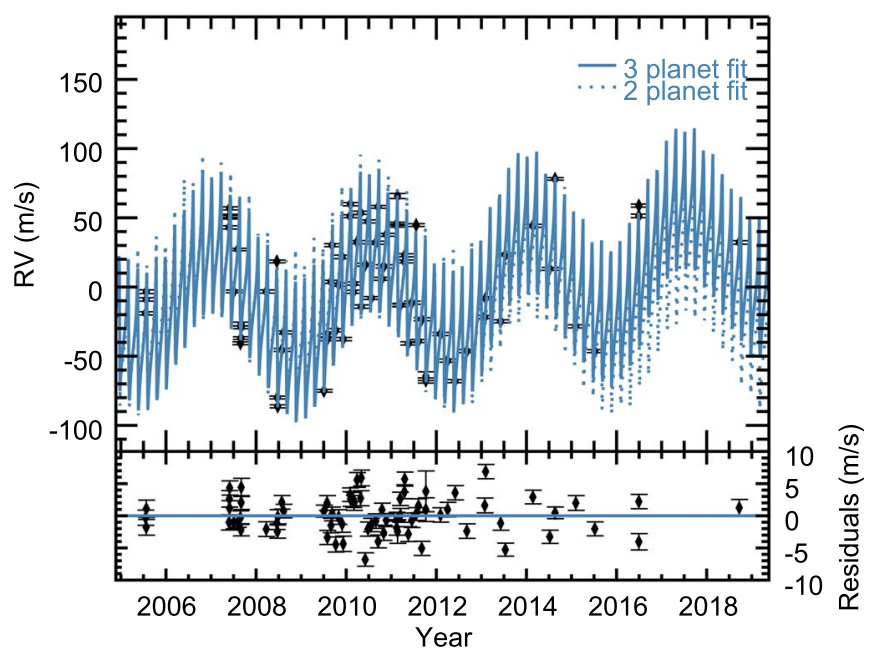

Figure 15. Full time series for HD 163607 and best three-planet fit. The new planet, HD $163607 \mathrm{~d}$ is the long-period trend that reaches minimum around 2009 and maximum in 2016, evident by the differing minima of the 1000 day planet). The difference between the two- and three-planet fits is not as easily seen in the time series. The bottom panel shows the residuals to the threeplanet fit.

\subsection{A Possible Jupiter Orbiting HD 207077}

HD 207077 is a G8 subgiant with $V=8.24, B-V=0.870$ (Wenger et al. 2000). It has effective temperature $T_{\text {eff }}=5067 \mathrm{~K}$, and surface gravity $\log g=3.27$. A summary of its stellar parameters can be found in Table 2 .

It has observations starting in 2007 that span roughly $8 \mathrm{yr}$. HD 207077 was previously identified in Butler et al. (2017) as having a planet candidate. The best-fit orbital solution yields a $m_{\mathrm{p}} \sin i=1.16 \pm 0.10 M_{\mathrm{Jup}}$ planet on a $606.3 \pm 3.8$ day period, with eccentricity, $e=0.204 \pm 0.099$. The full set of orbital parameters are given in Table 3 . Figure 21 shows the time series of HD 207077 b, with the initial and final periodogram for this system. Despite passing all of our planet detection thresholds, we are conservative for this star due to poor phase coverage and have classified it as a candidate. Continued observations will likely add to the periodogram peak and result in better phase coverage and confirm the planet status. We additionally note that this star has a relatively high RV rms compared to similar stars (J. K. Luhn et al. 2018, in preparation). Comparing the expected level of stellar jitter from similar stars to the measured RV rms for this star indicates further evidence for an unsubtracted planet in the data that is responsible for the artificially high $\mathrm{RV}$ rms.

\subsection{A Possible Additional Planet Orbiting HD 33142}

HD 33142 is a K0 subgiant (Wenger et al. 2000) with one previously known planet (Johnson et al. 2011). It has a $V$ magnitude $V=7.96, B-V=0.945$, effective temperature $T_{\text {eff }}=4978 \mathrm{~K}$, and $\log g=3.40$. It has a mass of $1.41 M_{\odot}$. Additional stellar properties can be found in Table 2. There are 40 observations spanning about $8 \mathrm{yr}$, and we make use of the additional seven points since the initial publication. The original fit for this planet in Johnson et al. (2011) noted unusually high jitter in this star and claimed evidence of a planet near 900 days, which we show in the periodogram with the residuals to our best fit to HD $33142 \mathrm{~b}$ in the right panel of Figure 22 . As can be seen, we find evidence for a second planet near 800 days. Refitting the RVs with a two-planet fit starting with a second planet near 800 days resulted in an improvement in the reduced $\chi^{2}$ from 38 to 19 . The minimum mass for HD $33142 \mathrm{c}$ is $0.59 \pm 0.10 M_{\text {Jup }}$ and it has a period of 809 days and eccentricity $e=0.16$. See Table 3 for more orbital and planet parameters and uncertainties. The time series for the second planet (HD 33142c) is shown in Figure 22 with the full time series and two-planet fit shown in Figure 23. This planet candidate does not meet our detection threshold $D>10$ with $D=9.4$. Additionally, it is not clear from the phase curve nor the full time series that the second planet is indeed present and with further observations we should know for certain.

\section{Nonplanetary Companions to Subgiant Stars}

A number of stars in our sample of subgiants showed evidence of stellar binary companions $\left(m \sin i>13 M_{\mathrm{Jup}}\right)$. All 

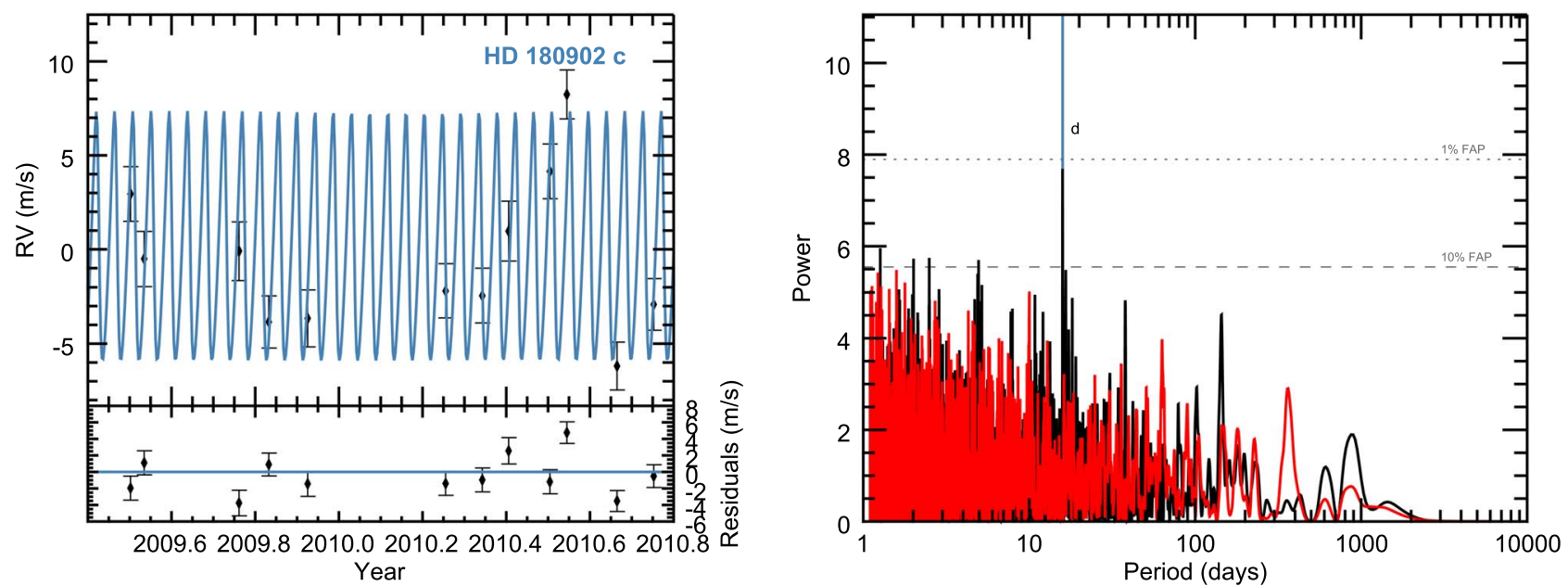

Figure 16. Residuals after subtracting out the previously discovered planet HD $180902 \mathrm{~b}$ (period $P=510.9$ days, eccentricity $e=0.107$, and minimum mass $m_{\mathrm{p}} \sin i=1.685 M_{\mathrm{Jup}}$ ) and the best-fit Keplerian for the stellar binary HD $180902 \mathrm{~B}$ (period $P=5880$ days, eccentricity $e=0.107$, and minimum mass $m_{\mathrm{p}} \sin i=98.7 M_{\mathrm{Jup}}$ ), showing evidence of a second planet. (Left) Time series for HD $180902 \mathrm{c}$ with period 15.9058 days, eccentricity $e=0.28$, and minimum mass $m_{\mathrm{p}} \sin i=0.099 M_{\mathrm{Jup}}$. Due to the short period, the time series on the left has been zoomed in on a high-density region of observations, but Figure 18 shows the full set of observations for this star with the longer period stellar binary companion. The phase curve for this planet is shown in Figure 17. (Right) Periodogram before (black) and after (red) subtracting the 15 day signal for HD $180902 \mathrm{c}$.

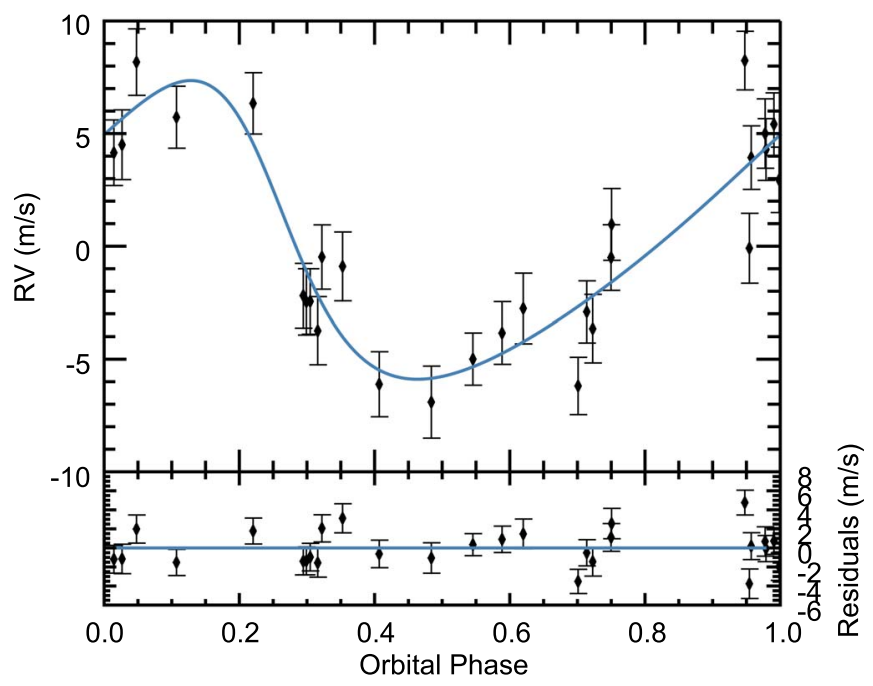

Figure 17. Phase curve for HD $180902 \mathrm{c}$ with period 15 days.

binaries are listed in Table 3 and their time series can be seen in Figure 24. In the following paragraphs, we describe those with minimum mass less than $100 M_{\text {Jup }}$, as these are likely brown dwarf candidates and of special interest to the exoplanet community.

\subsection{A $22 M_{\text {Jup }}$ Brown Dwarf Orbiting HD 125390}

HD 125390 is a G7 star (Wenger et al. 2000) with $V$-band magnitude 8.21 , effective temperature $T_{\text {eff }}=4850 \mathrm{~K}$, and surface gravity $\log g=3.13$. It has a mass of $1.12 M_{\odot}$ and has 15 observations over a $6 \mathrm{yr}$ span. We present the discovery of a $22.16 \pm 0.96 M_{\text {Jup }}$ brown dwarf companion to this star with period $1756.2 \pm 3.9$ days. The rest of the orbital parameters can be found in Table 3. The time series for this companion is shown in Figure 24.

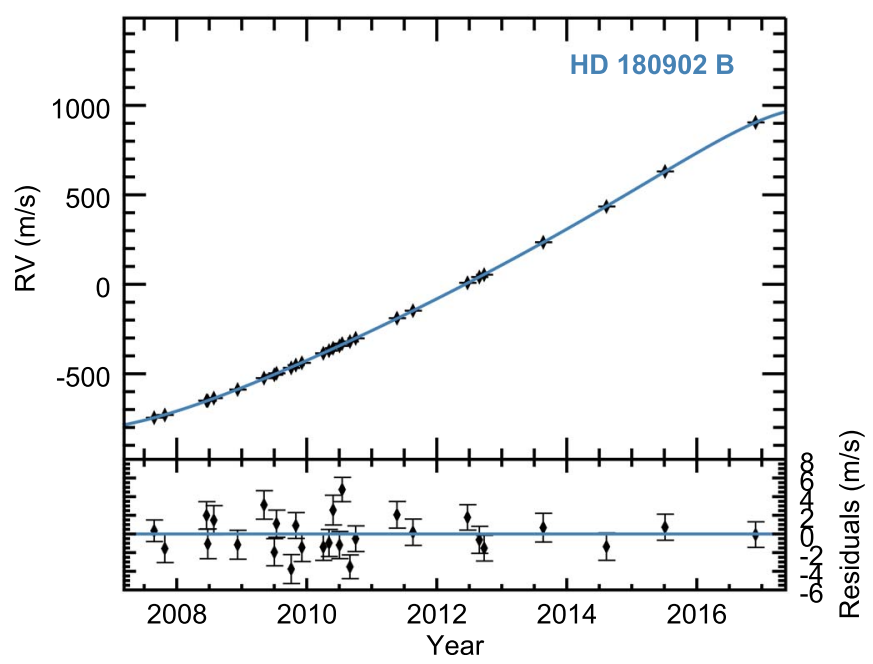

Figure 18. Time series for HD 180902 A, showing the signal due to the stellar companion HD 180902 B, where the signals from HD $180902 \mathrm{~b}$ and $\mathrm{c}$ have been subtracted out. Here we show that the previously identified linear trend has some curvature and can be fit by a Keplerian with period 5880 days; however, the phase coverage is incomplete, so errors for estimated parameters are large. For now, it appears to be a low-mass star or brown dwarf.

\section{2. $H D 148284$}

HD 148284 is a K0 star (Wenger et al. 2000) with $V$-band magnitude 9.01, effective temperature $T_{\text {eff }}=5572 \mathrm{~K}$, and surface gravity $\log g=3.97$. It has a mass of $1.02 M_{\odot}$ and has 30 observations over an $11 \mathrm{yr}$ span. We present the discovery of a $34.5 \pm 0.96 M_{\text {Jup }}$ brown dwarf companion to this star with period $339.302 \pm 0.026$ days. The rest of the orbital parameters can be found in Table 3 . The time series for this companion is shown in Figure 24.

\section{3. $H D 214823$}

HD 214823 is a G0 star (Wenger et al. 2000) with $V$-band magnitude 8.07 , effective temperature $T_{\text {eff }}=5933 \mathrm{~K}$, and 

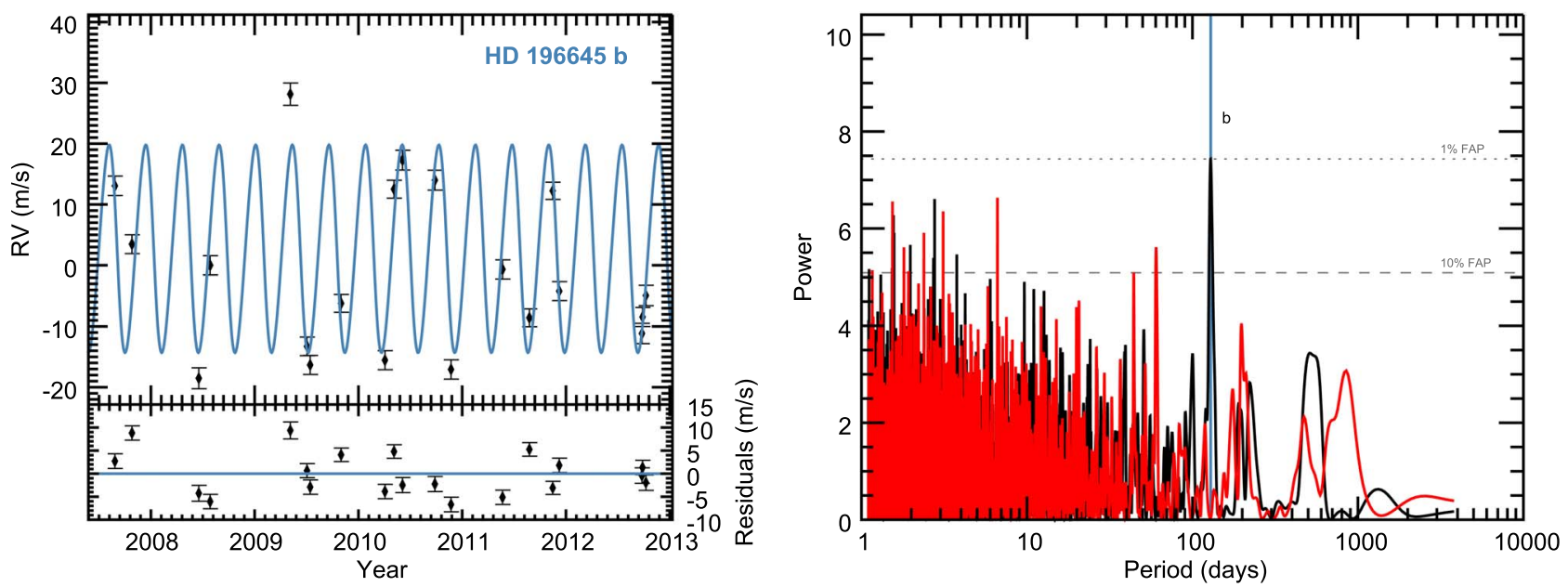

Figure 19. (Left) Time series and best-fit orbital solution for HD $196645 \mathrm{~b}$, with a 128.94 day period, eccentricity 0.106 , and minimum mass $0.497 M_{\text {Jup }}$. The residuals are shown in the bottom panel, which have an $\mathrm{RV} \mathrm{rms}=4.7 \mathrm{~m} \mathrm{~s}^{-1}$. The remaining best-fit parameters can be found in Table 3 . The phase curve can be seen in Figure 20. (Right) Periodogram of HD 196645 RV data before (black) and after (red) subtracting the best-fit planet parameters for HD 196645 b. The vertical line indicates the best-fit period of HD $196645 \mathrm{~b}$.

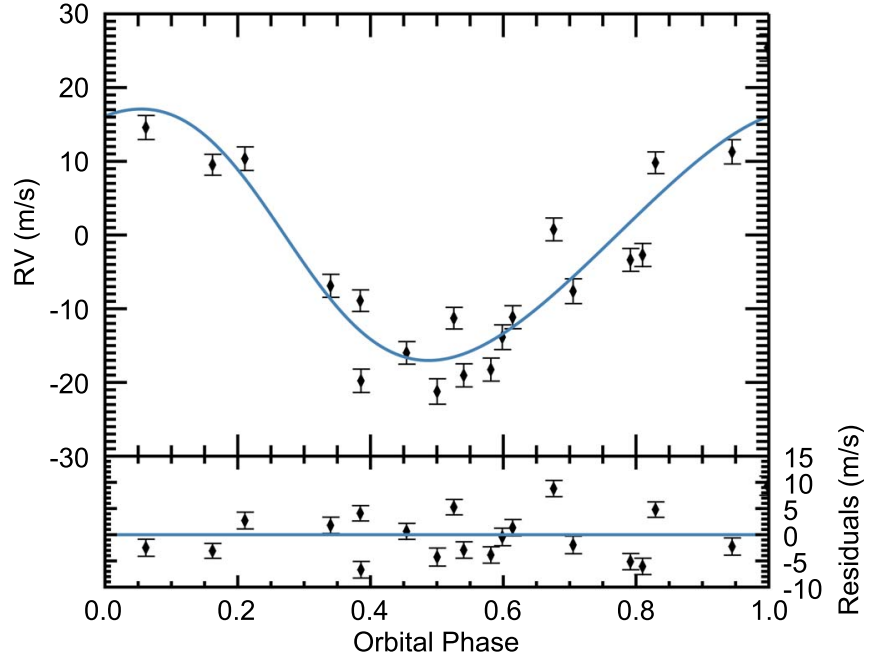

Figure 20. Phase-folded velocities of the best-fit solution for HD $196645 \mathrm{~b}$.

surface gravity $\log g=3.92$. It has a mass of $1.31 M_{\odot}$ and has 28 observations over an $11 \mathrm{yr}$ span. We present the discovery of a $20.56 \pm 0.32 M_{\text {Jup }}$ brown dwarf companion to this star with period $1853.9 \pm 1.6$ days. The rest of the orbital parameters and uncertainties can be found in Table 3. The time series for this companion is shown in Figure 24.

\subsection{Brown Dwarf Candidate Orbiting HD 180902}

We have already remarked on this system in Section 4.4; however, we wish to make a final remark here. The minimum mass for the stellar companion HD $180902 \mathrm{~B}\left(98.7 \pm 7.6 M_{\text {Jup }}\right)$ places it in as a candidate brown dwarf. However, the orbit for this companion is poorly constrained and so it is likely that the mass could substantially change with continued observations.

\section{Transit Times, Probabilities, Depths, and Durations of CPS Subgiants with Known RV Planets}

Here we describe the transit parameters for subgiant companions used in this work, all of which are are given in Table 5. At typical separations of planets in this paper (1-2 au), the average value for the transit probability is roughly $2.2 \% .^{12}$ For the 60 planets in Table 5 it is likely that 1 or 2 will in fact transit. Observing a planet in transit provides a wealth of additional information about the system, most notably the size and mass (without the $\sin i$ dependence) of the planet, which provides a bulk density for the planet. As an example, KELT$11 \mathrm{~b}$ (also referred to as HD 93396) is a highly inflated planet -which we know only because we have mass and radius information from the combined RV and transit data-on a short-period orbit around a subgiant star (Pepper et al. 2017). With transit observations of RV planets on similarly close orbits around subgiants we can understand how unique inflated planets like KELT-11 b are and whether the increased insolation from the evolved subgiant host plays a role in inflating the planet. Furthermore, transits provide a modelindependent measure of the stellar density, which combined with the radius from parallax would produce a modelindependent stellar mass (Seager \& Mallén-Ornelas 2003). Using Gaia parallaxes, reasonable SED measurements, and precise transits, errors in the stellar radius would be $1 \%$, and mass errors 3\% (Beatty et al. 2017).

In order to determine the best transit parameters, we refit all RV data for known subgiants. The reasons for this were twofold: (1) several of these planets have additional observations since their published discovery, and (2) updated stellar parameters from Brewer et al. (2016) allow for more accurate planet masses and reduced uncertainties in predicted transit time durations. All best-fit orbital parameters in this work were obtained using the IDL RVLIN package (Wright et al. 2009) in conjunction with the BOOTTRAN (Wang et al. 2012) package, which works with RVLIN and uses the bootstrapping technique to find best-fit parameters and uncertainties of RV fits. The best-fit orbital solutions for each planet is given in Table 3 and we include all RV observations used in this work in Table 4. Once we fit the RV data and obtained new orbital parameters, the final remaining step was to estimate radii in order to calculate estimated transit parameters. We used the Python

\footnotetext{
${ }^{12}$ We have excluded binary companions and only included planet companions in this rough calculation. We have also done a literature search for detections of transits (null or positive) for those in our sample with transit probability $>10 \%$ and have removed those from this calculation.
} 

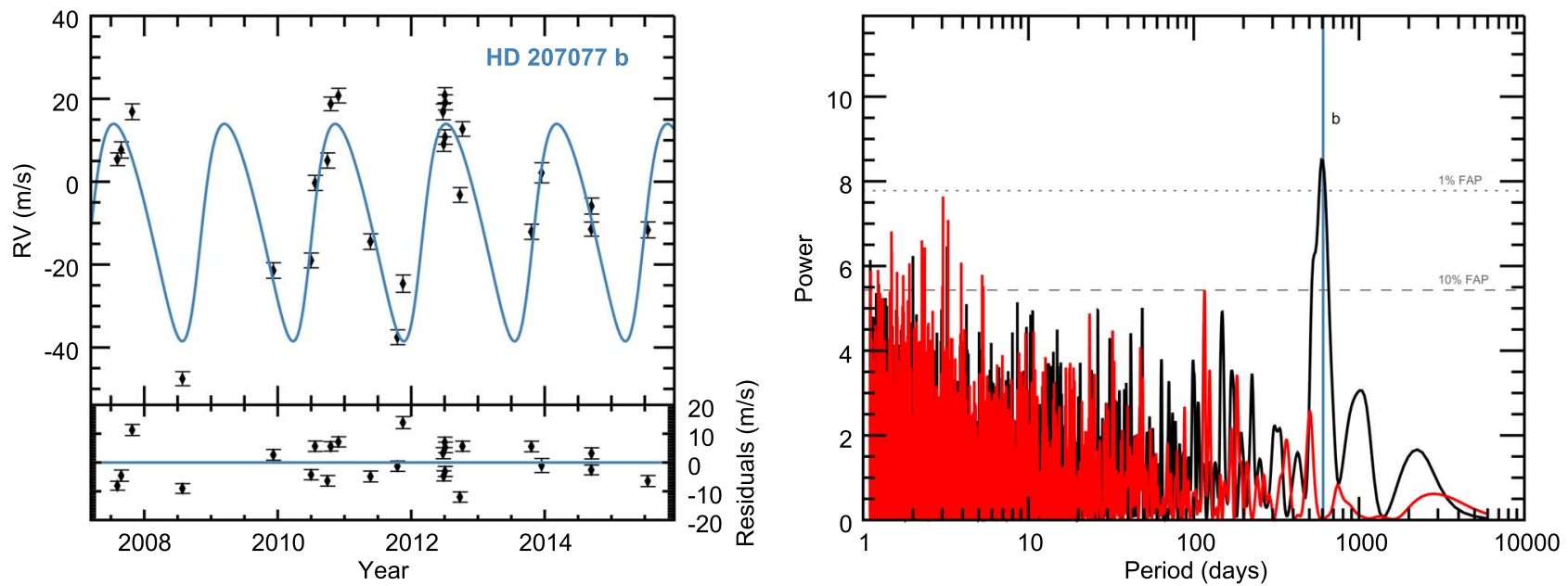

Figure 21. (Left) Time series and best-fit orbital solution for $\mathrm{HD} 207077 \mathrm{~b}$, which has period $P=606.3$ days, eccentricity $e=0.204$, and minimum mass $m_{\mathrm{p}} \sin i=1.16$ $M_{\text {Jup. }}$ The residuals are shown in the bottom panel, which have an RV rms $=6.7 \mathrm{~m} \mathrm{~s}^{-1}$. The remaining best-fit parameters can be found in Table 3. (Right) Periodogram of HD 207077 RV data before (black) and after (red) subtracting the best-fit planet parameters for HD $207077 \mathrm{~b}$. The vertical line indicates the best-fit period of HD $207077 \mathrm{~b}$.
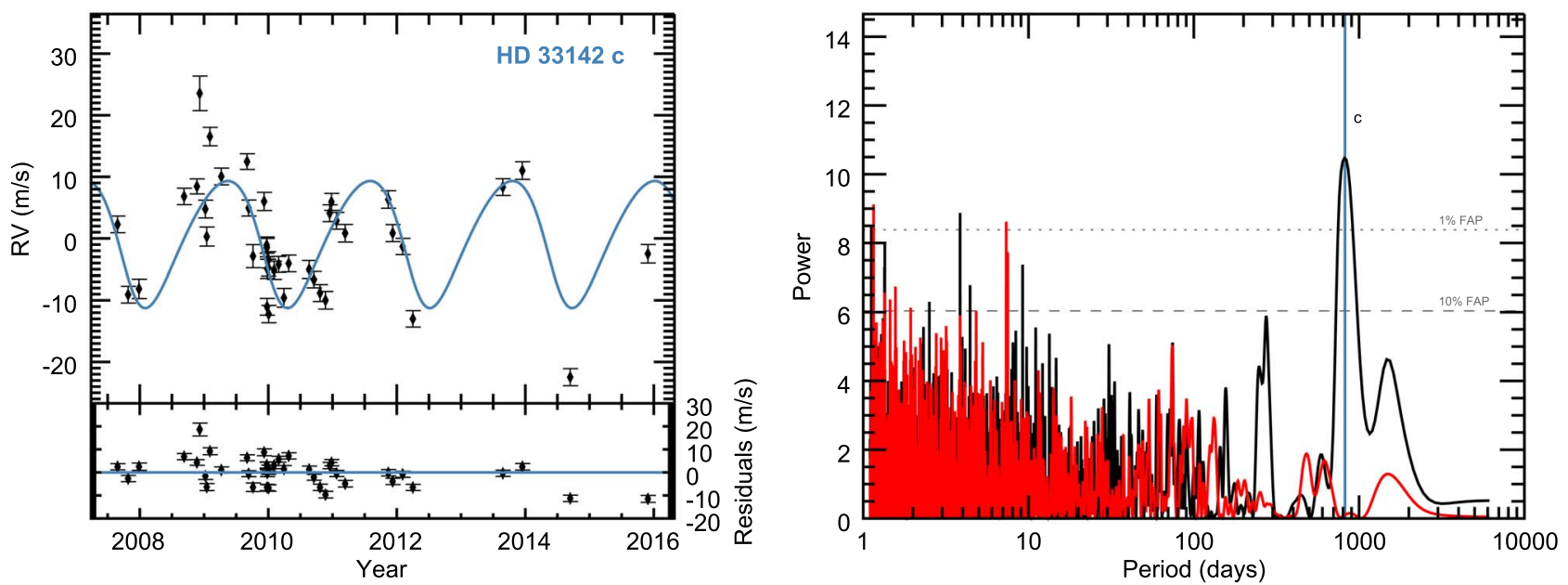

Figure 22. Residuals after subtracting out the previously discovered planet HD $33142 \mathrm{~b}$ (period $P=326.0$ days, eccentricity $e=0.066$, and minimum mass $m_{\mathrm{p}} \sin i=1.306 M_{\text {Jup }}$ ), showing evidence of a second planet. (Left) Time series for HD $33142 \mathrm{c}$ with period 809 days, eccentricity $e=0.16$, and minimum mass $m_{\mathrm{p}} \sin i=0.59 M_{\text {Jup }}$. (Right) Periodogram after subtracting out the best-fit orbital parameters for the previously discovered planet HD $33142 \mathrm{~b}$ (black) and the final periodogram after subtracting out the best two-planet fit (red). Note the black peak at 800 days, the starting guess used in the two-planet fit.

code FORECAST (Chen \& Kipping 2017) to estimate radii from minimum masses provided by the RV fits. We note that our transit calculations are meant to be estimates and, as such, we merely used the mean radius from FORECAST and do not include radius errors. ${ }^{13}$ The BOOTTRAN package also outputs the Barycentric Julian Date (BJD) of the next $n$ times of inferior conjunction and their uncertainties. For simplicity we use the terms "conjunction time" and "transit time" synonymously despite the fact that the planet may not actually transit. Using BOOTTRAN, we predict the next three transits for each planet starting after 2018 October 1 and report those with uncertainties as carried through by BOOTTRAN in Julian Date (columns 3-8). They require space-based observations because the majority of these planets are at 1-2 au around inflated subgiants so their transit depths will likely be difficult to observe with ground-based telescopes, and transit durations and ingress/egress durations will be rather long, again posing a difficulty for ground-based transits. Despite the short

\footnotetext{
$\overline{13}$ Typical radius errors were $0.2 R_{\text {Jup }}$, which results in less than $0.01 \%$ uncertainty in the transit probabilities.
}

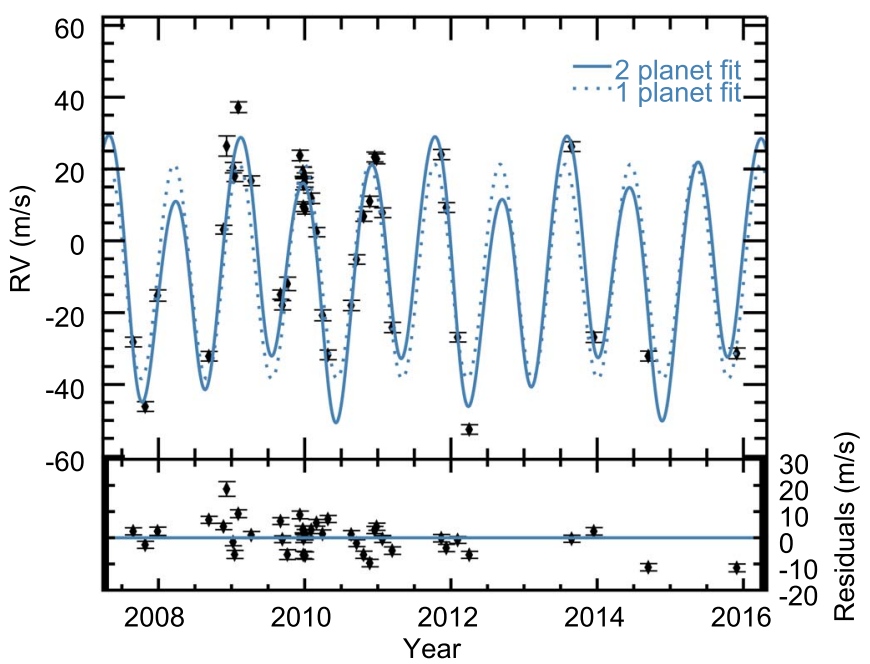

Figure 23. Full time series for HD 33142 and best two-planet fit. The dotted line shows for reference the best one-planet fit. The bottom panel shows the residuals to the best two-planet fit. 

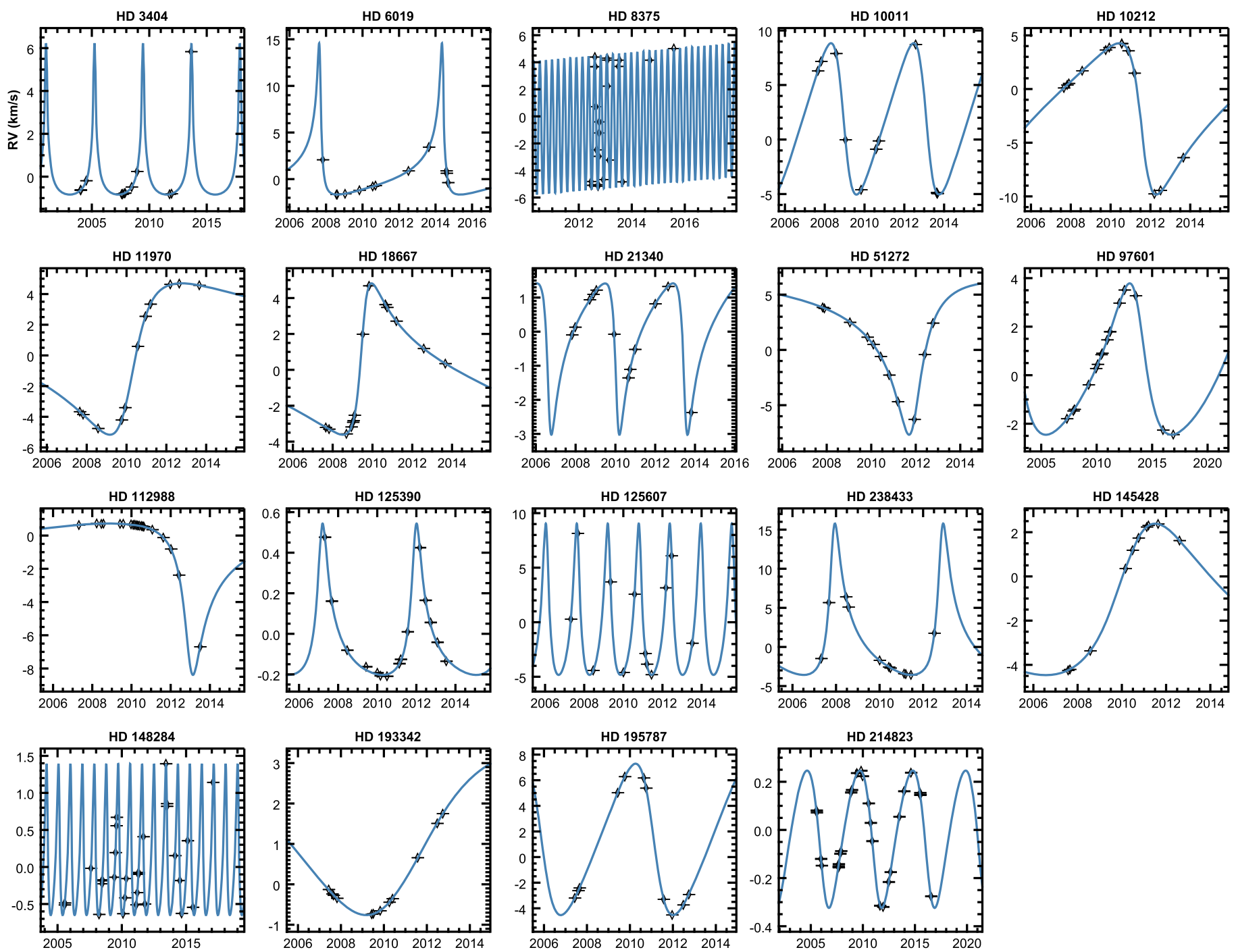

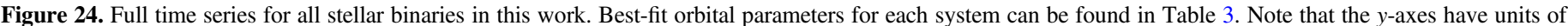
kilometers per second. Vertical error bars are added for clarity as in previous figures. The horizontal marks do not show error in time.

Table 4

Radial Velocities

\begin{tabular}{lcccc}
\hline \hline Star & $\begin{array}{c}\text { Date } \\
(\mathrm{JD}-2440,000) \\
(1)\end{array}$ & $\begin{array}{c}\mathrm{RV} \\
\left(\mathrm{m} \mathrm{s}^{-1}\right) \\
(3)\end{array}$ & $\begin{array}{c}\sigma_{\mathrm{RV}} \\
\left(\mathrm{m} \mathrm{s}^{-1}\right)\end{array}$ & $\begin{array}{c}\text { Tel } \\
(4)\end{array}$ \\
\hline HD 10697 & 10367.062 & -35.57 & 1.28 & Keck \\
HD 10697 & 10461.806 & -90.39 & 1.22 & Keck \\
HD 10697 & 10715.068 & -104.39 & 1.17 & Keck \\
HD 10697 & 10716.098 & -103.92 & 1.17 & Keck \\
HD 10697 & 10806.863 & -43.35 & 1.25 & Keck \\
HD 10697 & 10837.732 & -37.86 & 1.37 & Keck \\
HD 10697 & 10838.708 & -31.17 & 1.07 & Keck \\
\hline
\end{tabular}

(This table is available in its entirety in machine-readable form.)

photometric baseline from surveys like TESS ( $\sim 27$ days for the shortest), they may manage to catch one of these planets in transit. Furthermore, the longer period RV planets have large uncertainties on their predicted transit times, which would pose further problems for ground-based observing. We calculate the transit parameters as given in Seager (2010), including the full a priori transit probability

$$
\tau_{\mathrm{pr}}=\frac{\left(R_{\star}+R_{\mathrm{p}}\right)}{a} \frac{(1+e \sin \omega)}{1-e^{2}},
$$

(see also Seagroves et al. 2003; Barnes 2007) which is given in column 9. True a posteriori probabilities (Stevens \& Gaudi 2013) can be computed with knowledge of the underlying mass function for planets orbiting subgiants with comparable periods and host star masses. Since the exoplanet mass function has negative slope, this means our transit probabilities as given in Equation (2) are slightly underestimated. The transit depth is simply

$$
\tau_{\text {depth }}=\left(\frac{R_{\mathrm{p}}}{R_{\star}}\right)^{2}
$$

and is given in column 10. We also calculate transit and ingress/egress durations, which are given by

$$
\tau_{\text {dur }}=\frac{P}{\pi} \arcsin \left(\frac{R_{\star}}{a \sqrt{\left(1+\frac{R_{\mathrm{p}}}{R_{\star}}\right)^{2}}}\right)
$$


Table 5

Transit Parameters for Known Planets around Subgiants

\begin{tabular}{|c|c|c|c|c|c|c|c|c|c|c|c|c|c|c|}
\hline Star & $\begin{array}{l}\text { Com } \\
\text { (2) }\end{array}$ & $\begin{array}{c}\tau_{1} \\
\text { (JD) } \\
\text { (3) }\end{array}$ & $\begin{array}{l}\sigma_{1} \\
\text { (JD) } \\
(4)\end{array}$ & $\begin{array}{l}\tau_{2} \\
(\mathrm{JD}) \\
(5)\end{array}$ & $\begin{array}{l}\sigma_{2} \\
\text { (JD) } \\
\text { (6) }\end{array}$ & $\begin{array}{c}\tau_{3} \\
(\mathrm{JD}) \\
(7)\end{array}$ & $\begin{array}{l}\sigma_{3} \\
(\mathrm{JD}) \\
(8)\end{array}$ & $\begin{array}{l}\tau_{\mathrm{pr}} \\
(\%) \\
(9)\end{array}$ & $\begin{array}{l}\tau_{\text {depth }} \\
(\mathrm{ppm}) \\
(10)\end{array}$ & $\begin{array}{l}\tau_{\text {dur }} \\
(\text { hr) } \\
(11)\end{array}$ & $\begin{array}{l}\Delta \tau_{12} \\
(\mathrm{hr}) \\
(12)\end{array}$ & $\begin{array}{c}\tau_{1} \\
\text { (yyyy mm dd) } \\
(13)\end{array}$ & $\begin{array}{c}\tau_{2} \\
\text { (yyyy mm dd) } \\
(14)\end{array}$ & $\begin{array}{c}\tau_{3} \\
\text { (yyyy mm dd) } \\
(15)\end{array}$ \\
\hline HD 10697 & $\mathrm{~b}$ & 2458961.13 & 6.46 & 2460036.82 & 7.17 & 2461112.51 & 7.91 & 0.46 & 4360.578 & 30.9724 & 1.9186 & 2020 Apr 21 & 2023 Apr 2 & 2026 Mar 13 \\
\hline \multirow{2}{*}{$\begin{array}{l}\text { HD } 38529^{\mathrm{a}} \\
\ldots\end{array}$} & b & 2458405.55 & 0.12 & 2458419.86 & 0.12 & 2458434.17 & 0.12 & $13.44^{\mathrm{a}}$ & 2582.275 & 7.9461 & 0.3837 & 2018 Oct 14 & 2018 Oct 28 & 2018 Nov 11 \\
\hline & $\mathrm{c}$ & 2458889.11 & 6.20 & 2461025.19 & 9.31 & 2463161.28 & 12.42 & 0.43 & 2023.902 & 47.3599 & 2.0389 & 2020 Feb 9 & 2025 Dec 15 & 2031 Oct 21 \\
\hline HD 114613 & $\mathrm{~b}$ & 2458570.08 & 194.59 & 2462572.23 & 288.82 & 2466574.38 & 394.43 & 0.22 & 3208.864 & 61.2665 & 3.2845 & 2019 Mar 27 & 2030 Mar 11 & 2041 Feb 23 \\
\hline HD 117176 & $\mathrm{~b}$ & 2458457.10 & 0.18 & 2458573.79 & 0.18 & 2458690.48 & 0.19 & 2.31 & 3843.355 & 15.8918 & 0.9276 & $2018 \operatorname{Dec} 4$ & 2019 Mar 31 & $2019 \mathrm{Jul} 25$ \\
\hline \multirow{2}{*}{$\begin{array}{l}\text { HD } 159868 \\
\ldots\end{array}$} & b & 2458487.74 & 24.49 & 2459671.82 & 31.03 & 2460855.89 & 37.79 & 0.46 & 3349.915 & 40.0036 & 2.1887 & 2019 Jan 4 & 2022 Apr 2 & 2025 Jun 29 \\
\hline & $\mathrm{c}$ & 2458662.80 & 12.74 & 2459013.76 & 13.50 & 2459364.73 & 14.29 & 0.86 & 3738.315 & 32.9151 & 1.8965 & 2019 Jun 28 & 2020 Jun 13 & 2021 May 30 \\
\hline HD 175541 & $\mathrm{~b}$ & 2458484.93 & 8.74 & 2458783.36 & 9.10 & 2459081.79 & 9.48 & 2.26 & 977.176 & 43.0429 & 1.3046 & 2019 Jan 1 & 2019 Oct 26 & 2020 Aug 20 \\
\hline HD 190228 & $\mathrm{~b}$ & 2459235.07 & 5.57 & 2460378.57 & 7.12 & 2461522.06 & 8.68 & 1.23 & 2271.500 & 25.5255 & 1.1612 & $2021 \mathrm{Jan} 20$ & 2024 Mar 9 & 2027 Apr 26 \\
\hline HD 1502 & $\mathrm{~b}$ & 2458557.95 & 10.45 & 2458986.43 & 11.52 & 2459414.90 & 12.61 & 1.81 & 675.349 & 56.3972 & 1.4284 & 2019 Mar 15 & 2020 May 16 & 2021 Jul 19 \\
\hline HD 3404 & * & 2459669.59 & 5.87 & 2461213.13 & 5.68 & 2462756.66 & 5.55 & 0.85 & 7472.735 & 29.4967 & 2.3470 & 2022 Mar 31 & 2026 Jun 21 & 2030 Sep 12 \\
\hline HD 4313 & $\mathrm{~b}$ & 2458725.41 & 8.63 & 2459081.63 & 9.47 & 2459437.84 & 10.33 & 2.47 & 584.958 & 49.8151 & 1.1763 & 2019 Aug 29 & 2020 Aug 20 & 2021 Aug 11 \\
\hline \multirow{2}{*}{$\begin{array}{l}\text { HD } 5319 \\
\ldots\end{array}$} & $\mathrm{b}$ & 2458827.91 & 10.46 & 2459465.05 & 11.69 & 2460102.20 & 12.93 & 1.30 & 961.450 & 58.1012 & 1.7473 & 2019 Dec 10 & $2021 \mathrm{Sep} 7$ & 2023 Jun 6 \\
\hline & $\mathrm{c}$ & 2459200.27 & 18.50 & 2460072.48 & 23.85 & 2460944.69 & 29.46 & 0.87 & 1004.424 & 78.9995 & 2.4268 & 2020 Dec 16 & 2023 May 7 & 2025 Sep 26 \\
\hline HD 5608 & $\mathrm{~b}$ & 2458994.82 & 28.82 & 2459774.69 & 33.12 & 2460554.56 & 37.51 & 1.22 & 594.445 & 80.4063 & 1.9137 & 2020 May 25 & $2022 \mathrm{Jul} 14$ & 2024 Sep 1 \\
\hline HD 6019 & * & 2459283.41 & 0.34 & 2461740.42 & 0.33 & 2464197.42 & 0.42 & 2.91 & 7244.043 & 51.6680 & 4.0526 & 2021 Mar 9 & 2027 Nov 30 & 2034 Aug 22 \\
\hline HD 8375 & * & 2458434.53 & 0.23 & 2458518.50 & 0.24 & 2458602.47 & 0.25 & 4.11 & 2293.321 & 26.8375 & 1.2261 & 2018 Nov 12 & $2019 \mathrm{Feb} 3$ & 2019 Apr 28 \\
\hline HD 10011 & * & 2459367.22 & 3.64 & 2460885.54 & 4.80 & 2462403.86 & 5.96 & 1.12 & 19522.233 & 65.4003 & 8.0175 & 2021 Jun 1 & $2025 \mathrm{Jul} 29$ & 2029 Sep 24 \\
\hline HD 10212 & * & 2459097.32 & 14.84 & 2462450.28 & 30.39 & 2465803.25 & 45.95 & 1.00 & 22553.972 & 74.5061 & 9.7283 & 2020 Sep 4 & 2029 Nov 9 & 2039 Jan 14 \\
\hline HD 10442 & b & 2458803.59 & 37.22 & 2459835.89 & 43.99 & 2460868.18 & 51.44 & 0.43 & 4105.661 & 43.7453 & 2.6342 & 2019 Nov 16 & 2022 Sep 13 & $2025 \mathrm{Jul} 11$ \\
\hline HD 11970 & * & 2461234.25 & 114.19 & 2469737.55 & 253.50 & 2478240.86 & 392.64 & 0.16 & 23684.597 & 266.2444 & 35.5096 & $2026 \mathrm{Jul} 12$ & 2049 Oct 23 & $2073 \mathrm{Feb} 2$ \\
\hline HD 13167 & $\mathrm{~b}$ & 2460962.95 & 98.75 & 2463575.46 & 98.45 & 2466187.97 & 101.56 & 0.18 & 2509.603 & 106.8638 & 5.0980 & 2025 Oct 14 & 2032 Dec 8 & $2040 \mathrm{Feb} 3$ \\
\hline HD 14787 & $\mathrm{~b}$ & 2458429.05 & 45.36 & 2459105.65 & 52.84 & 2459782.24 & 60.41 & 1.38 & 655.045 & 75.1774 & 1.8760 & 2018 Nov 6 & 2020 Sep 13 & $2022 \mathrm{Jul} 21$ \\
\hline HD 18015 & $\mathrm{~b}$ & 2460468.05 & 152.62 & 2462746.29 & 217.80 & 2465024.54 & 285.76 & 0.34 & 1471.475 & 78.8912 & 2.9144 & 2024 Jun 6 & 2030 Sep 1 & 2036 Nov 27 \\
\hline HD 18667 & * & 2460779.46 & 13.81 & 2465155.06 & 26.48 & 2469530.66 & 39.24 & 0.25 & 6995.873 & 252.0085 & 19.4513 & $2025 \mathrm{Apr} 13$ & 2037 Apr 6 & 2049 Mar 30 \\
\hline \multirow{2}{*}{$\begin{array}{l}\text { HD } 18742 \\
\ldots\end{array}$} & b & 2458913.70 & 86.02 & 2459680.11 & 110.79 & 2460446.53 & 136.03 & 1.34 & 543.356 & 79.2323 & 1.8048 & 2020 Mar 5 & $2022 \mathrm{Apr} 10$ & 2024 May 16 \\
\hline & $\mathrm{c}$ & 2458828.96 & 968.46 & 2459687.69 & 1004.79 & 2460546.41 & 1041.14 & 1.22 & 569.978 & 83.5374 & 1.9478 & 2019 Dec 11 & $2022 \mathrm{Apr} 18$ & 2024 Aug 23 \\
\hline HD 21340 & * & 2458942.89 & 6.76 & 2460192.58 & 9.24 & 2461442.27 & 11.71 & 2.40 & 645.813 & 63.2127 & 1.5666 & $2020 \mathrm{Apr} 3$ & 2023 Sep 5 & 2027 Feb 5 \\
\hline HD 28678 & b & 2458525.45 & 13.23 & 2458905.67 & 14.72 & 2459285.90 & 16.23 & 2.97 & 377.848 & 66.7229 & 1.2721 & 2019 Feb 10 & $2020 \mathrm{Feb} 26$ & 2021 Mar 12 \\
\hline HD 30856 & $\mathrm{~b}$ & 2458419.15 & 61.31 & 2459266.62 & 80.60 & 2460114.09 & 100.08 & 1.16 & 819.205 & 72.9918 & 2.0310 & 2018 Oct 27 & $2021 \mathrm{Feb} 21$ & 2023 Jun 18 \\
\hline \multirow{2}{*}{$\begin{array}{l}\text { HD } 33142 \\
\ldots\end{array}$} & $\mathrm{b}$ & 2458542.97 & 12.35 & 2458868.93 & 13.42 & 2459194.88 & 14.52 & 2.03 & 844.941 & 46.7382 & 1.3201 & 2019 Feb 28 & 2020 Jan 20 & 2020 Dec 11 \\
\hline & $\mathrm{c}$ & 2459201.07 & 224.26 & 2460009.99 & 485.33 & 2460818.91 & 677.24 & 1.23 & 903.456 & 57.6308 & 1.6817 & 2020 Dec 17 & 2023 Mar 6 & 2025 May 23 \\
\hline HD 38801 & $\mathrm{~b}$ & 2458945.18 & 8.46 & 2459631.94 & 9.74 & 2460318.70 & 11.06 & 0.67 & 2330.606 & 39.2068 & 1.8056 & 2020 Apr 5 & $2022 \mathrm{Feb} 21$ & $2024 \operatorname{Jan} 9$ \\
\hline HD 45410 & $\mathrm{~b}$ & 2459029.89 & 33.20 & 2459964.16 & 41.37 & 2460898.43 & 49.72 & 1.24 & 568.684 & 79.0858 & 1.8420 & 2020 Jun 29 & 2023 Jan 19 & 2025 Aug 10 \\
\hline HD 51272 & * & 2461027.52 & 664.54 & 2466686.99 & 1340.99 & 2472346.45 & 2017.44 & 0.65 & 13116.036 & 210.3041 & 21.6101 & $2025 \operatorname{Dec} 18$ & 2041 Jun 16 & 2056 Dec 13 \\
\hline HD 72490 & $\mathrm{~b}$ & 2459092.41 & 50.54 & 2459950.47 & 61.31 & 2460808.53 & 72.24 & 1.37 & 634.563 & 76.3301 & 1.8755 & 2020 Aug 30 & 2023 Jan 5 & 2025 May 13 \\
\hline HD 73534 & $\mathrm{~b}$ & 2458536.19 & 66.60 & 2460286.17 & 88.79 & 2462036.14 & 111.76 & 0.44 & 2472.393 & 54.5783 & 2.5853 & 2019 Feb 21 & 2023 Dec 7 & 2028 Sep 21 \\
\hline \multirow{2}{*}{$\begin{array}{l}\text { HD } 75784 \\
\ldots\end{array}$} & $\mathrm{b}$ & 2458550.23 & 12.45 & 2458891.42 & 13.36 & 2459232.62 & 14.29 & 1.58 & 1441.043 & 42.0341 & 1.5372 & 2019 Mar 7 & 2020 Feb 11 & 2021 Jan 18 \\
\hline & c & 2464151.96 & 6775.66 & 2472003.35 & 9117.63 & 2479854.75 & 11295.54 & 0.18 & 1226.221 & 142.9796 & 4.8374 & 2034 Jul 8 & 2056 Jan 5 & 2077 Jul 5 \\
\hline HD $88133^{\mathrm{a}}$ & $\mathrm{b}$ & 2458395.69 & 0.04 & 2458399.10 & 0.04 & 2458402.52 & 0.04 & $22.89^{\mathrm{a}}$ & 2376.247 & 5.7657 & 0.2657 & 2018 Oct 4 & 2018 Oct 7 & 2018 Oct 11 \\
\hline HD $93396^{\mathrm{b}}$ & $\mathrm{b}$ & 2458392.98 & 0.18 & 2458397.71 & 0.18 & 2458402.45 & 0.18 & $25.65^{\mathrm{b}}$ & 964.937 & 6.7779 & 0.2026 & 2018 Oct 1 & 2018 Oct 6 & 2018 Oct 11 \\
\hline HD 94834 & $\mathrm{~b}$ & 2459431.40 & 174.81 & 2461007.85 & 249.29 & 2462584.30 & 324.60 & 0.81 & 920.072 & 80.7779 & 2.3781 & 2021 Aug 4 & 2025 Nov 28 & 2030 Mar 23 \\
\hline \multirow{2}{*}{ HD 95089} & $\mathrm{~b}$ & 2459086.08 & 63.01 & 2460870.86 & 93.21 & 2462655.64 & 123.93 & 1.00 & 553.833 & 75.5569 & 1.7372 & 2020 Aug 24 & $2025 \mathrm{Jul} 14$ & 2030 Jun 3 \\
\hline & $\mathrm{c}$ & 2458827.04 & 205.76 & 2459291.39 & 208.43 & 2459755.75 & 211.27 & 1.63 & 628.965 & 70.1498 & 1.7162 & $2019 \operatorname{Dec} 9$ & 2021 Mar 17 & 2022 Jun 25 \\
\hline HD 96063 & b & 2458607.90 & 35.45 & 2458970.42 & 37.29 & 2459332.94 & 39.18 & 1.76 & 719.066 & 67.4129 & 1.7604 & 2019 May 4 & $2020 \mathrm{Apr} 30$ & 2021 Apr 28 \\
\hline HD 96167 & $\mathrm{~b}$ & 2458688.67 & 28.19 & 2459186.71 & 28.67 & 2459684.75 & 29.14 & 0.49 & 4524.795 & 56.1604 & 3.5396 & 2019 Jul 24 & 2020 Dec 3 & 2022 Apr 15 \\
\hline HD 97601 & * & 2460937.49 & 442.33 & 2465078.54 & 834.37 & 2469219.59 & 1227.61 & 0.56 & 6822.725 & 93.2152 & 7.1121 & 2025 Sep 18 & 2037 Jan 20 & 2048 May 23 \\
\hline HD 98219 & $\mathrm{~b}$ & 2458637.96 & 14.02 & 2459071.72 & 15.83 & 2459505.49 & 17.68 & 1.75 & 728.688 & 57.9542 & 1.5232 & 2019 Jun 3 & 2020 Aug 10 & 2021 Oct 17 \\
\hline HD 99706 & $\mathrm{~b}$ & 2458729.97 & 135.98 & 2459571.05 & 167.08 & 2460412.13 & 198.52 & 1.69 & 534.082 & 69.4327 & 1.5683 & 2019 Sep 3 & 2021 Dec 22 & 2024 Apr 11 \\
\hline HD 102956 & $\mathrm{~b}$ & 2458393.61 & 0.09 & 2458400.11 & 0.09 & 2458406.60 & 0.09 & 26.16 & 807.999 & 13.9966 & 0.3821 & 2018 Oct 2 & 2018 Oct 8 & 2018 Oct 15 \\
\hline HD 106270 & b & 2458868.48 & 31.51 & 2460756.22 & 45.92 & 2462643.96 & 60.93 & 0.41 & 1913.123 & 54.3065 & 2.2758 & 2020 Jan 19 & 2025 Mar 21 & 2030 May 22 \\
\hline HD 108863 & $\mathrm{~b}$ & 2458503.72 & 22.95 & 2458941.38 & 25.67 & 2459379.04 & 28.42 & 2.06 & 455.595 & 69.6541 & 1.4556 & 2019 Jan 20 & 2020 Apr 1 & 2021 Jun 13 \\
\hline
\end{tabular}


Table 5

(Continued)

\begin{tabular}{|c|c|c|c|c|c|c|c|c|c|c|c|c|c|c|}
\hline Star & $\begin{array}{l}\text { Com } \\
\text { (2) }\end{array}$ & $\begin{array}{l}\tau_{1} \\
(\mathrm{JD}) \\
(3)\end{array}$ & $\begin{array}{l}\sigma_{1} \\
(\mathrm{JD}) \\
(4)\end{array}$ & $\begin{array}{l}\tau_{2} \\
(\mathrm{JD}) \\
(5)\end{array}$ & $\begin{array}{l}\sigma_{2} \\
(\mathrm{JD}) \\
(6)\end{array}$ & $\begin{array}{c}\tau_{3} \\
(\mathrm{JD}) \\
(7)\end{array}$ & $\begin{array}{c}\sigma_{3} \\
(\mathrm{JD}) \\
(8)\end{array}$ & $\begin{array}{l}\tau_{\mathrm{pr}} \\
(\%) \\
(9)\end{array}$ & $\begin{array}{c}\tau_{\text {depth }} \\
(\mathrm{ppm}) \\
(10)\end{array}$ & $\begin{array}{l}\tau_{\text {dur }} \\
(\mathrm{hr}) \\
(11)\end{array}$ & $\begin{array}{l}\Delta \tau_{12} \\
(\mathrm{hr}) \\
(12)\end{array}$ & $\begin{array}{c}\tau_{1} \\
\text { (yyyy } \mathrm{mm} d \mathrm{dd}) \\
(13)\end{array}$ & $\begin{array}{c}\tau_{2} \\
\text { (yyyy } \mathrm{mm} \text { dd) } \\
(14)\end{array}$ & $\begin{array}{c}\tau_{3} \\
\text { (yyyy mm dd) } \\
(15)\end{array}$ \\
\hline HD 112988 & * & 2461931.23 & 512.60 & 2467708.70 & 1034.71 & 2473486.17 & 1556.84 & 1.11 & 4836.263 & 96.7614 & 6.2916 & 2028 Jun 8 & 2044 Apr 3 & 2060 Jan 27 \\
\hline HD 125390 & * & 2459610.29 & 11.10 & 2461366.53 & 13.24 & 2463122.77 & 16.01 & 1.23 & 293.634 & 127.7546 & 2.1523 & 2022 Jan 30 & 2026 Nov 22 & 2031 Sep 13 \\
\hline HD 125607 & * & 2458440.62 & 0.65 & 2459019.16 & 0.75 & 2459597.70 & 0.86 & 1.91 & 8429.183 & 50.7931 & 4.2711 & 2018 Nov 18 & 2020 Jun 18 & 2022 Jan 18 \\
\hline HD 131496 & b & 2458858.64 & 64.26 & 2459755.11 & 79.51 & 2460651.58 & 94.89 & 1.26 & 790.488 & 61.8007 & 1.6900 & $2020 \mathrm{Jan} 10$ & 2022 Jun 24 & 2024 Dec 7 \\
\hline HD 238433 & * & 2460094.04 & 3.23 & 2461910.93 & 3.45 & 2463727.81 & 3.67 & 0.95 & 20244.924 & 128.7559 & 16.0378 & 2023 May 29 & 2028 May 19 & 2033 May 10 \\
\hline HD 142091 & b & 2459098.22 & 32.95 & 2460383.06 & 46.94 & 2461667.89 & 61.15 & 0.86 & 661.820 & 88.0362 & 2.2080 & 2020 Sep 5 & 2024 Mar 13 & 2027 Sep 19 \\
\hline HD 145428 & * & 2462668.38 & 397.89 & 2468280.63 & 744.03 & 2473892.87 & 1090.24 & 0.38 & 4043.203 & 249.4931 & 14.9158 & 2030 Jun 15 & 2045 Oct 27 & 2061 Mar 9 \\
\hline HD 148284 & * & 2458524.00 & 0.19 & 2458863.30 & 0.22 & 2459202.60 & 0.24 & 1.10 & 5465.489 & 14.7810 & 1.0175 & 2019 Feb 9 & 2020 Jan 14 & $2020 \operatorname{Dec} 19$ \\
\hline HD 152581 & $\mathrm{~b}$ & 2458997.97 & 25.32 & 2459684.46 & 29.86 & 2460370.94 & 34.45 & 1.48 & 587.046 & 77.2530 & 1.8274 & 2020 May 28 & 2022 Apr 14 & 2024 Mar 1 \\
\hline HD 163607 & b & 2458398.49 & 0.41 & 2458473.71 & 0.42 & 2458548.93 & 0.43 & 9.42 & 5468.759 & 5.3829 & 0.3706 & 2018 Oct 6 & 2018 Dec 21 & 2019 Mar 6 \\
\hline$\ldots$ & c & 2459545.27 & 17.76 & 2460817.26 & 22.21 & 2462089.25 & 26.66 & 0.34 & 4911.309 & 38.6675 & 2.5324 & 2021 Nov 26 & 2025 May 21 & 2028 Nov 13 \\
\hline$\ldots$ & $\mathrm{d}$ & 2486985.03 & 55697.98 & 2523485.03 & 69120.33 & 2559985.03 & 82553.73 & 0.05 & 4403.954 & 95.3278 & 5.9325 & 2097 Jan 11 & 2196 Dec 18 & 2296 Nov 24 \\
\hline HD 180053 & $\mathrm{~b}$ & 2458409.70 & 7.29 & 2458623.42 & 7.73 & 2458837.13 & 8.19 & 2.51 & 923.284 & 34.8194 & 1.0267 & 2018 Oct 18 & 2019 May 19 & $2019 \operatorname{Dec} 19$ \\
\hline HD 180902 & b & 2458519.95 & 9.27 & 2459030.83 & 10.59 & 2459541.71 & 11.94 & 1.44 & 907.231 & 55.2221 & 1.6146 & 2019 Feb 5 & 2020 Jun 30 & 2021 Nov 23 \\
\hline$\ldots$ & * & 2458811.85 & 432.62 & 2464687.86 & 864.08 & 2470563.88 & 1492.06 & 0.41 & 854.855 & 89.2750 & 2.5361 & 2019 Nov 24 & 2035 Dec 26 & $2052 \operatorname{Jan} 27$ \\
\hline & $\mathrm{c}$ & 2458407.32 & 7.73 & 2458423.22 & 7.73 & 2458439.13 & 7.73 & 19.37 & 193.241 & 13.1158 & 0.1792 & 2018 Oct 15 & 2018 Oct 31 & 2018 Nov 16 \\
\hline HD 181342 & b & 2458512.87 & 29.15 & 2459077.01 & 32.59 & 2459641.16 & 36.15 & 1.38 & 671.517 & 62.1357 & 1.5694 & 2019 Jan 29 & 2020 Aug 15 & 2022 Mar 2 \\
\hline HD $185269^{\mathrm{a}}$ & $\mathrm{b}$ & 2458397.66 & 0.12 & 2458404.50 & 0.12 & 2458411.34 & 0.12 & $13.79^{\mathrm{a}}$ & 4158.104 & 6.4653 & 0.3906 & 2018 Oct 6 & 2018 Oct 13 & 2018 Oct 19 \\
\hline HD 192699 & b & 2458513.21 & 8.92 & 2458854.15 & 9.70 & 2459195.09 & 10.50 & 2.16 & 786.785 & 47.6008 & 1.2987 & 2019 Jan 29 & 2020 Jan 5 & 2020 Dec 11 \\
\hline HD 193342 & ${ }^{*}$ & 2462813.37 & 14955.48 & 2472466.91 & 30914.85 & 2482120.44 & 46834.52 & 0.27 & 4388.284 & 227.6026 & 14.1406 & 2030 Nov 7 & 2057 Apr 13 & 2083 Sep 17 \\
\hline HD 195787 & * & 2459422.83 & 1.40 & 2461332.09 & 1.68 & 2463241.35 & 2.00 & 1.02 & 12042.169 & 82.6617 & 8.1740 & $2021 \mathrm{Jul} 27$ & 2026 Oct 18 & $2032 \operatorname{Jan} 9$ \\
\hline HD 196645 & $\mathrm{~b}$ & 2458473.62 & 11.52 & 2458602.56 & 11.90 & 2458731.50 & 12.29 & 3.38 & 1484.883 & 26.7608 & 0.9928 & 2018 Dec 21 & 2019 Apr 29 & 2019 Sep 5 \\
\hline HD 200964 & b & 2458926.10 & 21.24 & 2459532.43 & 24.92 & 2460138.76 & 28.65 & 1.61 & 652.605 & 65.1233 & 1.6221 & 2020 Mar 17 & 2021 Nov 13 & $2023 \mathrm{Jul} 13$ \\
\hline$\ldots$ & $\mathrm{c}$ & 2458767.79 & 28.41 & 2459620.25 & 35.37 & 2460472.71 & 42.41 & 1.01 & 673.304 & 95.5130 & 2.4156 & 2019 Oct 11 & $2022 \mathrm{Feb} 9$ & 2024 Jun 11 \\
\hline HD 206610 & b & 2458959.86 & 19.45 & 2459633.01 & 22.42 & 2460306.17 & 25.46 & 1.61 & 409.932 & 89.3480 & 1.7730 & $2020 \mathrm{Apr} 20$ & $2022 \mathrm{Feb} 22$ & $2023 \operatorname{Dec} 27$ \\
\hline HD 207077 & $\mathrm{~b}$ & 2458749.90 & 35.94 & 2459356.21 & 40.23 & 2459962.51 & 44.74 & 1.10 & 1049.793 & 72.6645 & 2.2804 & 2019 Sep 23 & 2021 May 21 & 2023 Jan 18 \\
\hline HD 210702 & $\mathrm{~b}$ & 2458551.16 & 8.31 & 2458905.26 & 8.89 & 2459259.36 & 9.48 & 2.04 & 643.242 & 55.5278 & 1.3734 & 2019 Mar 8 & $2020 \mathrm{Feb} 25$ & $2021 \mathrm{Feb} 13$ \\
\hline HD 212771 & b & 2458403.22 & 15.54 & 2458783.94 & 16.78 & 2459164.67 & 18.04 & 2.19 & 541.166 & 58.8900 & 1.3387 & 2018 Oct 11 & 2019 Oct 27 & 2020 Nov 11 \\
\hline HD 214823 & ${ }^{*}$ & 2459227.73 & 4.42 & 2461081.61 & 5.87 & 2462935.49 & 7.37 & 0.36 & 2962.866 & 38.0524 & 1.9643 & $2021 \mathrm{Jan} 13$ & $2026 \mathrm{Feb} 10$ & 2031 Mar 9 \\
\hline HD 4917 & $\mathrm{~b}$ & 2458760.37 & 14.45 & 2459160.92 & 15.97 & 2459561.46 & 17.50 & 1.96 & 628.655 & 65.7449 & 1.6080 & 2019 Oct 3 & 2020 Nov 7 & 2021 Dec 12 \\
\hline$\ldots$ & c & 2459102.46 & 308.26 & 2459923.50 & 319.62 & 2460744.55 & 331.14 & 2.16 & 640.833 & 52.9620 & 1.3076 & 2020 Sep 9 & $2022 \operatorname{Dec} 10$ & 2025 Mar 10 \\
\hline$\ldots$ & d & 2459441.36 & 704.84 & 2460534.02 & 741.14 & 2461626.68 & 777.38 & 0.85 & 669.968 & 112.9123 & 2.8488 & 2021 Aug 14 & 2024 Aug 11 & 2027 Aug 9 \\
\hline
\end{tabular}

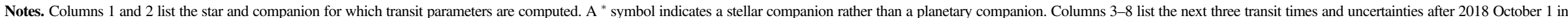

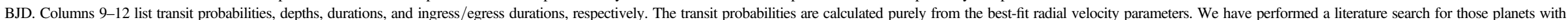
transit probabilities greater than 10\%; those that are known to transit or not transit are marked accordingly. Finally, columns 13-15 list the date of the next three transits in yyyy mm dd format (UTC) for easy reference.

${ }^{a}$ Planet is known to not transit.

${ }^{b}$ Planet is known to transit.

(This table is available in machine-readable form.) 


$$
\Delta \tau_{12}=\frac{P R_{\mathrm{p}}}{\pi a}
$$

and can be found in columns 11 and 12 . We note that the duration equations have been simplified to assume a circular orbit and exclude grazing transits, which are acceptable for first-order calculations. Finally, the three transit times given in columns 3, 5, and 7 are listed again in YYYY MM DD format (UTC) for quick reference.

While the large majority of planets have probabilities $1 \%$ $2 \%$, we highlight three planets with transit probabilities greater than 9\%: HD $102956 \mathrm{~b}(26.16 \%)$, HD $180902 \mathrm{c}(19.37 \%)$, and HD $163607 \mathrm{~b}(9.55 \%) .{ }^{14}$ These are all planets with periods less than about 75 days, relatively short for evolved stars of intermediate mass. With the exception of HD $180902 \mathrm{c}$, which is a large hot Neptune candidate, they also have predicted depths of $\sim 800 \mathrm{ppm}$ or more, which makes these more amenable for ground-based observations than the other planets. Although $800 \mathrm{ppm}$ is still a challenge for ground-based observing, it has been demonstrated on midclass telescopes with diffuser-assisted photometry (Stefansson et al. 2017). As short-period RV planets, their periods are more tightly constrained, and so transit time uncertainties are relatively small ( $\ll 1$ day, with the exception of HD $180902 \mathrm{c}$ ). We emphasize that the majority of the planets around subgiants have semimajor axes of 1 au or more, which in combination with the large stellar radii leads to long transit durations, small transit depths, and lower transit probabilities. ${ }^{15}$ In addition, many of the uncertainties in the midtransit times are on the order of 10 days (anywhere from 13 days to 150 days), which makes hunting for them with ground-based telescopes difficult. These factors are what make these planets more amenable to a space-based survey like TESS, which may incidentally catch a single transit for one of these planets.

Refitting each known planet as we did means that Table 3, which contains the best-fit orbital parameters for all CPS subgiant stars with known planets, lists the most precise and up-to-date orbital solutions for these planets.

\section{Summary and Conclusion}

In this work, we presented the discovery of 15 new planetary signals around subgiant stars, 8 of which are planet candidates requiring further observations, increasing the number of known RV planets around subgiants by $\sim 15 \%$ (25\% including the candidates). Of special importance are possibly the first threeplanet systems around subgiant stars, HD 163607 and HD 4917. As observations on subgiants continue and more longperiod planets are discovered, these systems will be useful in determining how multiplanet systems evolve as their host star leaves the main sequence. We lastly note several stellar companions of brown dwarfs to nearly solar mass size and provide orbital parameters for these systems as well.

\footnotetext{
$\overline{14}$ We have not included HD 93396 (KELT-11), which we list as having a 26\% transit probability, because it is known to transit and was in fact discovered via transits (Pepper et al. 2017). We have also excluded HD 88133 b, which has transit probability $22.89 \%$ as it has been found to be nontransiting (Piskorz et al. 2016). Additional planets excluded from this list as they have been found to be nontransiting include HD 185269 b (Johnson et al. 2006; Moutou et al. 2006) with $13 \%$ transit probability, and HD 38529 b (Henry et al. 2013) with $13 \%$ transit probability.

15 The increased stellar radii actually helps to inflate the transit probabilities, but the large separations ultimately account for the low probabilities.
}

In this work, we have calculated transit parameters (transit times, probabilities, depths, and durations, see Table 5) for all known planets around CPS subgiant stars $(3<\log g<4)$. We find that three planets have relatively high transit probabilities $(\gtrsim 10 \%)$. These planets have the best chance of having a transit observed from a ground-based telescope. The remaining 50 planets in general all have lower transit probabilities $(1 \%-2 \%)$, longer transit durations $(\sim 50 \mathrm{hr})$, smaller transit depths (of order $500 \mathrm{ppm}$ ), and more uncertain transit times (tens of days). The combination of these factors indicates that these are challenging to observe from the ground, but instead will make good targets for future space-based missions like TESS. In predicting transit parameters, we have made use of additional RV observations since the planets' initial discoveries and updated stellar parameters to refit all planets, resulting in updated orbital parameters for all planets (Table 3).

We would like to thank the anonymous referee whose suggestions have greatly enhanced this paper. We would like to thank Thomas Beatty and Arpita Roy for their discussions and helpful comments. Many of the new planets and planet candidates in this work have come to fruition with the last several years of observations. These observations would not have taken place without Andrew Howard shepherding the observations and extending the time baseline for these systems. We also wish to thank Alan Reyes for his help observing. We also wish to thank Scott Gaudi for pointing out that our transit probabilities are a priori transit probabilities and Dan Stevens for clarification on how the a posteriori probabilities would differ. The authors wish to recognize and acknowledge the very significant cultural role and reverence that the summit of Maunakea has always had within the indigenous Hawaiian community. We are most fortunate to have the opportunity to conduct observations from this mountain.

This research has made use of the SIMBAD database, operated at CDS, Strasbourg, France; the Exoplanet Orbit Database and the Exoplanet Data Explorer at exoplanets.org; and of NASA's Astrophysics Data System Bibliographic Services. This material is based upon work supported by the National Science Foundation Graduate Research Fellowship Program under grant No. DGE1255832.

Software: RVLIN (Wright et al. 2009), BOOTTRAN (Wang et al. 2012), FORECAST (Chen \& Kipping 2017).

\section{ORCID iDs}

Jacob K. Luhn (10 https://orcid.org/0000-0002-4927-9925 Fabienne A. Bastien (iD https://orcid.org/0000-00027243-1921

Jason T. Wright (iD https://orcid.org/0000-0001-6160-5888 Andrew W. Howard (i) https://orcid.org/0000-00018638-0320

Howard Isaacson (iD https://orcid.org/0000-0002-0531-1073

\section{References}

Baines, E. K., Armstrong, J. T., \& van Belle, G. T. 2013, ApJL, 771, L17 Barnes, J. W. 2007, PASP, 119, 986

Bastien, F. A., Stassun, K. G., \& Pepper, J. 2014, ApJL, 788, L9

Beatty, T. G., Stevens, D. J., Collins, K. A., et al. 2017, AJ, 154, 25

Borucki, W. J., Koch, D., Basri, G., et al. 2010, Sci, 327, 977

Borucki, W. J., Koch, D. G., Basri, G., et al. 2011, ApJ, 736, 19

Bowler, B. P., Johnson, J. A., Marcy, G. W., et al. 2010, ApJ, 709, 396

Brewer, J. M., Fischer, D. A., Basu, S., Valenti, J. A., \& Piskunov, N. 2015, ApJ, 805, 126 
Brewer, J. M., Fischer, D. A., Valenti, J. A., \& Piskunov, N. 2016, ApJS, 225,32

Brewer, J. M., Fischer, D. A., Valenti, J. A., \& Piskunov, N. 2017, ApJS, 230, 12

Bryan, M. L., Knutson, H. A., Howard, A. W., et al. 2016, ApJ, 821, 89

Butler, R. P., Marcy, G. W., Williams, E., et al. 1996, PASP, 108, 500

Butler, R. P., Vogt, S. S., Laughlin, G., et al. 2017, AJ, 153, 208

Butler, R. P., Wright, J. T., Marcy, G. W., et al. 2006, ApJ, 646, 505

Chen, J., \& Kipping, D. 2017, ApJ, 834, 17

Ciceri, S., Lillo-Box, J., Southworth, J., et al. 2015, A\&A, 573, L5

Collins, K. A., Eastman, J. D., Beatty, T. G., et al. 2014, AJ, 147, 39

Dawson, R. I., \& Fabrycky, D. C. 2010, ApJ, 722, 937

Díaz, R. F., Ségransan, D., Udry, S., et al. 2016, A\&A, 585, A134

Diego, F., Charalambous, A., Fish, A. C., \& Walker, D. D. 1990, Proc. SPIE, 1235,562

Endl, M., Kürster, M., \& Els, S. 2000, A\&A, 362, 585

Fischer, D. A., \& Valenti, J. 2005, ApJ, 622, 1102

Ghezzi, L., Montet, B. T., \& Johnson, J. A. 2018, ApJ, 860, 109

Giguere, M. J., Fischer, D. A., Howard, A. W., et al. 2012, ApJ, 744, 4

Giguere, M. J., Fischer, D. A., Payne, M. J., et al. 2015, ApJ, 799, 89

Harakawa, H., Sato, B., Fischer, D. A., et al. 2010, ApJ, 715, 550

Henry, G. W., Kane, S. R., Wang, S. X., et al. 2013, ApJ, 768, 155

Howard, A. W., Johnson, J. A., Marcy, G. W., et al. 2010, ApJ, 721, 1467

Howard, A. W., Johnson, J. A., Marcy, G. W., et al. 2011, ApJ, 726, 73

Huber, D., Chaplin, W. J., Christensen-Dalsgaard, J., et al. 2013, ApJ, 767, 127

Ida, S., \& Lin, D. N. C. 2004, ApJ, 604, 388

Isaacson, H., \& Fischer, D. 2010, ApJ, 725, 875

Johnson, J. A., Aller, K. M., Howard, A. W., \& Crepp, J. R. 2010a, PASP, 122,905

Johnson, J. A., Butler, R. P., Marcy, G. W., et al. 2007a, ApJ, 670, 833

Johnson, J. A., Clanton, C., Howard, A. W., et al. 2011, ApJS, 197, 26

Johnson, J. A., Fischer, D. A., Marcy, G. W., et al. 2007b, ApJ, 665, 785

Johnson, J. A., Howard, A. W., Bowler, B. P., et al. 2010b, PASP, 122, 701

Johnson, J. A., Marcy, G. W., Fischer, D. A., et al. 2006, ApJ, 652, 1724

Johnson, J. A., Morton, T. D., \& Wright, J. T. 2013, ApJ, 763, 53

Johnson, J. A., \& Wright, J. T. 2013, arXiv:1307.3441

Jones, H. R. A., Paul Butler, R., Marcy, G. W., et al. 2002, MNRAS, 337, 1170

Jones, M. I., Jenkins, J. S., Brahm, R., et al. 2016, A\&A, 590, A38

Kane, S. R., Mahadevan, S., von Braun, K., Laughlin, G., \& Ciardi, D. R. 2009, PASP, 121, 1386

Kaufer, A., Stahl, O., Tubbesing, S., et al. 1999, Msngr, 95, 8

Lloyd, J. P. 2011, ApJL, 739, L49

Lloyd, J. P. 2013, ApJL, 774, L2
Morton, T. D., Bryson, S. T., Coughlin, J. L., et al. 2016, ApJ, 822, 86

Moutou, C., Loeillet, B., Bouchy, F., et al. 2006, A\&A, 458, 327

Noguchi, K., Aoki, W., Kawanomoto, S., et al. 2002, PASJ, 54, 855

Otor, O. J., Montet, B. T., Johnson, J. A., et al. 2016, AJ, 152, 165

Peek, K. M. G., Johnson, J. A., Fischer, D. A., et al. 2009, PASP, 121, 613

Pepper, J., Rodriguez, J. E., Collins, K. A., et al. 2017, AJ, 153, 215

Piskorz, D., Benneke, B., Crockett, N. R., et al. 2016, ApJ, 832, 131

Quinn, S. N., White, T. R., Latham, D. W., et al. 2015, ApJ, 803, 49

Ramsey, L. W., Adams, M. T., Barnes, T. G., et al. 1998, Proc. SPIE, 3352 34

Ricker, G. R., Winn, J. N., Vanderspek, R., et al. 2014, Proc. SPIE, 9143, 914320

Robinson, S. E., Laughlin, G., Vogt, S. S., et al. 2007, ApJ, 670, 1391

Rowe, J. F., Bryson, S. T., Marcy, G. W., et al. 2014, ApJ, 784, 45

Sato, B. 2005, JKAS, 38, 315

Sato, B., Izumiura, H., Toyota, E., et al. 2008, PASJ, 60, 539

Sato, B., Omiya, M., Harakawa, H., et al. 2012, PASJ, 64, 135

Schlaufman, K. C., \& Winn, J. N. 2013, ApJ, 772, 143

Seager, S. 2010, Exoplanets (Tucson, AZ: Univ. Arizona Press)

Seager, S., \& Mallén-Ornelas, G. 2003, ApJ, 585, 1038

Seagroves, S., Harker, J., Laughlin, G., Lacy, J., \& Castellano, T. 2003, PASP, 115,1355

Stefansson, G., Mahadevan, S., Hebb, L., et al. 2017, ApJ, 848, 9

Stello, D., Huber, D., Grundahl, F., et al. 2017, MNRAS, 472, 4110

Stevens, D. J., \& Gaudi, B. S. 2013, PASP, 125, 933

Tokovinin, A., Fischer, D. A., Bonati, M., et al. 2013, PASP, 125, 1336

Tull, R. G. 1998, Proc. SPIE, 3355, 387

Tull, R. G., MacQueen, P. J., Sneden, C., \& Lambert, D. L. 1995, PASP, 107,251

Valenti, J. A., Fischer, D., Marcy, G. W., et al. 2009, ApJ, 702, 989

Villaver, E., \& Livio, M. 2009, ApJL, 705, L81

Vogt, S. S. 1987, PASP, 99, 1214

Wang, S. X., Wright, J. T., Cochran, W., et al. 2012, ApJ, 761, 46

Wenger, M., Ochsenbein, F., Egret, D., et al. 2000, A\&AS, 143, 9

Wittenmyer, R. A., Endl, M., Cochran, W. D., Levison, H. F., \& Henry, G. W. 2009, ApJS, 182, 97

Wittenmyer, R. A., Horner, J., Tinney, C. G., et al. 2014, ApJ, 783, 103

Wittenmyer, R. A., Horner, J., Tuomi, M., et al. 2012, ApJ, 753, 169

Wittenmyer, R. A., Johnson, J., Wang, L., \& Endl, M. 2011, in AIP Conf. Ser 1331, Planetary Systems beyond the Main Sequence, ed. S. Schuh, H. Drechsel, \& U. Heber (Melville, NY: AIP), 117

Wright, J. T. 2005, PASP, 117, 657

Wright, J. T., Upadhyay, S., Marcy, G. W., et al. 2009, ApJ, 693, 1084 